\title{
Duality invariance and higher derivatives
}

\author{
Camille Eloy $\odot,{ }^{1}$ Olaf Hohm, ${ }^{2}$ and Henning Samtleben $\odot^{1}$ \\ ${ }^{1}$ Université Lyon, Ens de Lyon, Université Claude Bernard, CNRS, Laboratoire de Physique, \\ F-69342 Lyon, France \\ ${ }^{2}$ Institute for Physics, Humboldt University Berlin, Zum Großen Windkanal 6, D-12489 Berlin, Germany
}

(Received 12 May 2020; accepted 9 June 2020; published 29 June 2020)

\begin{abstract}
We dimensionally reduce the spacetime action of bosonic string theory, and that of the bosonic sector of heterotic string theory after truncating the Yang-Mills gauge fields, on a $d$-dimensional torus including all higher-derivative corrections to first order in $\alpha^{\prime}$. A systematic procedure is developed that brings this action into a minimal form in which all fields except the metric carry only first-order derivatives. This action is shown to be invariant under $\mathrm{O}(d, d, \mathbb{R})$ transformations that acquire $\alpha^{\prime}$-corrections through a GreenSchwarz type mechanism. We prove that, up to a global prefactor, the first-order $\alpha^{\prime}$-corrections are uniquely determined by $\mathrm{O}(d, d, \mathbb{R})$ invariance.
\end{abstract}

DOI: $10.1103 /$ PhysRevD.101.126018

\section{INTRODUCTION}

String theory features the $T$-duality property according to which there is a nonlinear group action of $\mathrm{O}(d, d, \mathbb{Z})$ on $d$-dimensional toroidal backgrounds such that all backgrounds in one orbit are physically equivalent. When restricting to the massless fields for compactifications on tori, i.e., when performing dimensional reduction, this duality implies invariance under the continuous symmetry group $\mathrm{O}(d, d, \mathbb{R})$. For the two-derivative effective action this symmetry was first shown explicitly for the (cosmological) reduction to one dimension by Veneziano and Meissner in Refs. [1,2] and later generalized to arbitrary $d$ by Maharana and Schwarz [3].

It was proven by Sen, using closed string field theory, that the $\mathrm{O}(d, d, \mathbb{R})$ symmetry of dimensionally reduced theories is present to all orders in $\alpha^{\prime}$ [4], but it remains as a highly nontrivial problem to actually display this symmetry when higher-derivative $\alpha^{\prime}$-corrections are included. First significant progress was due to Meissner who investigated the dimensional reduction to one dimension including the four-derivative terms that appear in string theory to first order in $\alpha^{\prime}$ [5] (for earlier work on the heterotic string see Ref. [6]). He uncovered the expected $\mathrm{O}(d, d, \mathbb{R})$ symmetry, but this required a series of elaborate field redefinitions (that in particular cannot all originate from covariant field redefinitions before reduction). Subsequent work considered the reduction on a single circle [7] and reductions on a

Published by the American Physical Society under the terms of the Creative Commons Attribution 4.0 International license. Further distribution of this work must maintain attribution to the author(s) and the published article's title, journal citation, and DOI. Funded by SCOAP ${ }^{3}$. general torus but truncating out all "off-diagonal" field components [8]. In all these truncations there is a choice of field variables for which the $\mathrm{O}(d, d, \mathbb{R})$ transformations are undeformed, as is also suggested by string field theory [9]. In particular, this fact was used to classify all higherderivative corrections in cosmology that, somewhat surprisingly, only require (higher powers of) first-order time derivatives [10,11].

Recently, the higher-derivative $\alpha^{\prime}$-corrections of string theory have been the focus of attention in the framework of double field theory. Double field theory is a formulation featuring a manifest $\mathrm{O}(d, d, \mathbb{R})$ invariance before dimensional reduction by virtue of a generalized spacetime with doubled coordinates transforming covariantly under $\mathrm{O}(d, d, \mathbb{R})$ [12-15]. While the two-derivative double field theory can be written naturally in terms of a "generalized metric" that encodes the metric and $B$-field [cf. Eq. (2.9) below], there are obstacles when including higher derivatives that require a deformation of the framework (see Refs. [16-23]). It was proven in Refs. [24,25] that the general $\alpha^{\prime}$-corrections of bosonic and heterotic string theory cannot be written in terms of the generalized metric, so that in particular the $\mathrm{O}(d, d, \mathbb{R})$ transformations of double field theory get $\alpha^{\prime}$-deformed. Alternatively, one may set up a generalized frame formalism for which $\mathrm{O}(d, d, \mathbb{R})$ remains undeformed while the local frame transformations receive $\alpha^{\prime}$-corrections [19,24,25].

In this paper we complete the existing literature by giving the complete dimensionally reduced action for bosonic string theory to first order in $\alpha^{\prime}$, i.e., including all four-derivative terms, and prove its $\mathrm{O}(d, d, \mathbb{R})$ invariance, presenting results that have recently been announced in Ref. [26]. In particular, we prove that the first-order $\alpha^{\prime}$-corrections are uniquely determined by $\mathrm{O}(d, d, \mathbb{R})$ 
invariance, up to an overall constant whose value depends on the string theory under consideration. While this $\mathrm{O}(d, d, \mathbb{R})$ invariance is also implied by the existence of $\alpha^{\prime}$-deformed double field theory, whose dimensional reduction has already been explored in Ref. [22], until now it has not been systematically investigated whether some of the unexpected new features arising in double field theory also show up in the dimensional reduction of conventional (nonextended) theories, nor has the dimensionally reduced action been displayed in a sufficiently simplified form that allows for applications (and comparison with some of the earlier results cited above). To our surprise we find that there is no choice of field variables so that the full dimensionally reduced action can be written in terms of familiar $\mathrm{O}(d, d, \mathbb{R})$ covariant variables (such as the generalized metric); rather, a generalized Green-Schwarz mechanism is required under which the (external) singlet $B$-field acquires nontrivial transformations under $\mathrm{O}(d, d, \mathbb{R})$, hence implying that the $\mathrm{O}(d, d, \mathbb{R})$ action gets $\alpha^{\prime}$ deformed. This effect has been invisible in all truncations investigated so far, but it does mimic the situation in double field theory before reduction. Intriguingly, the $\alpha^{\prime}$-deformations needed in double field theory can thus not be blamed entirely on its novel geometric structure, but such deformations also emerge in completely conventional dimensional reductions.

On a technical level, the present investigation requires full control over all possible field redefinitions, both redefinitions that are covariant in the usual sense [i.e., $\mathrm{GL}(d)$ covariant] and covariant with respect to $\mathrm{O}(d, d, \mathbb{R})$. As one of the main technical results of this paper we present a fully systematic procedure to test $\mathrm{O}(d, d, \mathbb{R})$ invariance, generalizing that of Refs. $[10,11]$ to higher dimensions. One first dimensionally reduces the action as usual and then uses $\operatorname{GL}(d)$ covariant field redefinitions to bring the action into a form in which all fields apart from the metric appear only with first-order derivatives. Next, one employs $\mathrm{O}(d, d, \mathbb{R})$ covariant redefinitions in order to find the minimal set of $\mathrm{O}(d, d, \mathbb{R})$ invariant four-derivative terms, which then are decomposed under $\operatorname{GL}(d)$ with the aim to match with the dimensionally reduced terms. Our analysis applies to bosonic string theory but also to the bosonic sector of heterotic string theory after truncating out the Yang-Mills gauge fields, which still features a gravitational Chern-Simons form (due to the original Green-Schwarz mechanism).

The rest of the paper is organized as follows. In Sec. II, we review the dimensional reduction of the leading twoderivative action of the bosonic string, and its manifestly $\mathrm{O}(d, d, \mathbb{R})$ symmetric formulation revealed in Ref. [3]. In order to set up a systematic analysis of its higher-order corrections, we outline how to organize and fix the ambiguities related to partial integration and higher-order field redefinitions. In Sec. III, we present a general counting of independent higher-derivative terms upon modding out these ambiguities. At order $\alpha^{\prime}$, we construct an explicit 61-dimensional basis of independent $\mathrm{O}(d, d, \mathbb{R})$ invariant four-derivative terms, which is algebraic in first-order derivatives and the Riemann tensor. Section IV presents the explicit torus reduction of the four-derivative action of the bosonic string. In particular, we show how all secondorder derivatives in the reduced action can be eliminated by suitable field redefinitions. Comparing the result to our explicit basis, we show in Sec. V that apart from a single term the entire reduced action can be rewritten in terms of manifestly $\mathrm{O}(d, d, \mathbb{R})$ invariant terms. Restoring $\mathrm{O}(d, d, \mathbb{R})$ invariance of the full action then requires a Green-Schwarz type mechanism inducing a nontrivial $\mathrm{O}(d, d, \mathbb{R})$ transformation of order $\alpha^{\prime}$ of the 2-form $B_{\mu \nu}$. In Sec. VI, we embed this structure into a frame formalism in which the $\mathrm{O}(d, d, \mathbb{R})$ symmetry remains undeformed, while the local frame transformations acquire $\alpha^{\prime}$ deformations. Finally, in Sec. VII, we extend the analysis to the bosonic sector of heterotic supergravity and present its dimensionally reduced action in manifestly $\mathrm{O}(d, d, \mathbb{R})$ invariant form. The Appendixes collect a number of explicit technical results.

\section{TWO-DERIVATIVE ACTION AND SYSTEMATICS OF FIELD REDEFINITIONS}

A main goal of this paper is to compute the dimensional reduction of the bosonic string on a $d$-dimensional torus including the first order in $\alpha^{\prime}$ and to make the resulting $\mathrm{O}(d, d, \mathbb{R})$ symmetry manifest. In this section, we review the reduction of the two-derivative action and its manifestly $\mathrm{O}(d, d, \mathbb{R})$ symmetric formulation first exhibited in Ref. [3]. We then discuss its field equations and the systematics of nonlinear field redefinitions as a starting point for the subsequent systematic analysis of the higherorder corrections.

\section{A. Reduction and $\mathbf{O}(d, d, \mathbb{R})$ symmetry}

Let us start from the two-derivative effective action for the bosonic string in $D+d$ dimensions, with metric $\hat{g}_{\hat{\mu} \hat{\nu}}$, antisymmetric Kalb-Ramond field $\hat{B}_{\hat{\mu} \hat{\nu}}$, and dilaton $\hat{\phi}$ :

$\hat{I}_{0}=\int \mathrm{d}^{D+d} X \sqrt{-\hat{g}} e^{-\hat{\phi}}\left(\hat{R}+\partial_{\hat{\mu}} \hat{\phi} \partial^{\hat{\mu}} \hat{\phi}-\frac{1}{12} \hat{H}^{2}\right)$,

where indices $\hat{\mu}$ run over the $(D+d)$-dimensional space and $\hat{H}^{2}=\hat{H}^{\hat{\mu} \hat{\nu} \hat{\rho}} \hat{H}_{\hat{\mu} \hat{\nu} \hat{\rho}}$ with the field strength $\hat{H}_{\hat{\mu} \hat{\nu} \hat{\rho}}=$ $3 \partial_{[\hat{\mu}} \hat{B}_{\hat{\nu} \hat{\rho}]}$. To compactify on the spatial torus $\mathrm{T}^{d}$, we use the index split $X^{\hat{\mu}}=\left(x^{\mu}, y^{m}\right)$, with $\mu \in \llbracket 1, D \rrbracket, m \in \llbracket 1, d \rrbracket$ for curved indices, and $\{\hat{\alpha}\}=\{\alpha, a\}$, with $\alpha \in \llbracket 1, D \rrbracket$, $a \in \llbracket 1, d \rrbracket$ for flat indices, and drop the dependence of all fields on the internal coordinates $y^{m}$. For the metric $\hat{g}_{\hat{\mu} \hat{\nu}}$, we use the vielbein formalism and consider the standard Kaluza-Klein ansatz 


$$
\hat{e}_{\hat{\mu}}^{\hat{\alpha}}=\left(\begin{array}{cc}
e_{\mu}{ }^{\alpha} & A_{\mu}^{(1) n} E_{n}{ }^{a} \\
0 & E_{m}{ }^{a}
\end{array}\right),
$$

in terms of the $D$-dimensional vielbein $e_{\mu}{ }^{\alpha}$, Kaluza-Klein vector fields $A_{\mu}^{(1) m}$, and the internal vielbein $E_{m}{ }^{a}$. The metric $\hat{g}_{\hat{\mu} \hat{\nu}}=\hat{e}_{\hat{\mu}}{ }_{\hat{\alpha}} \eta_{\hat{\alpha} \hat{\beta}} \hat{e}_{\hat{\nu}}^{\hat{\beta}}$ then takes the form

$$
\hat{g}_{\hat{\mu} \hat{\nu}}=\left(\begin{array}{cc}
g_{\mu \nu}+A_{\mu}^{(1) p} G_{p q} A_{\nu}^{(1) q} & A_{\mu}^{(1) p} G_{p n} \\
G_{m p} A_{\nu}^{(1) p} & G_{m n}
\end{array}\right),
$$

where $g_{\mu \nu}=e_{\mu}{ }^{\alpha} \eta_{\alpha \beta} e_{\nu}{ }^{\beta}$ and $G_{m n}=E_{m}{ }^{a} \delta_{a b} E_{n}{ }^{b}$ denote the $D$-dimensional metric and the internal metric, respectively.

Similarly, the 2-form $\hat{B}_{\hat{\mu} \hat{\nu}}$, is parametrized as [3]

$\hat{B}_{\hat{\mu} \hat{\nu}}=\left(\begin{array}{cc}B_{\mu \nu}-A_{[\mu}^{(1) m} A_{\nu] m}^{(2)}+A_{\mu}^{(1) m} B_{m n} A_{\nu}^{(1) n} & A_{\mu n}^{(2)}-B_{n p} A_{\mu}^{(1) p} \\ -A_{\nu m}^{(2)}+B_{m p} A_{\nu}^{(1) p} & B_{m n}\end{array}\right)$,

in terms of $D$-dimensional scalars $B_{m n}=-B_{n m}$, vector fields $A_{\mu m}^{(2)}$, and a 2 -form $B_{\mu \nu}$. The lower-dimensional components of $\hat{H}_{\hat{\mu} \hat{\nu} \hat{\rho}}$ are defined using the standard Kaluza-Klein procedure [3]: first converting $\hat{H}$ to flat indices, block decomposing, and finally converting back to curved indices using the lower-dimensional blocks $e_{\mu}{ }^{\alpha}$ and $E_{m}{ }^{a}$. This amounts to converting a curved index $\hat{\mu}$ to a curved index $\mu$ using a contraction with $e_{\mu}{ }^{\alpha} \hat{e}_{\alpha}{ }^{\hat{\mu}}$ and to $m$ contracting with $E_{m}{ }^{a} \hat{e}_{\alpha}{ }^{\hat{\mu}}$, such that the resulting fields transform covariantly under internal diffeomorphisms. ${ }^{1}$ With Eq. (2.3), this leads to

$$
\left\{\begin{array}{l}
H_{\mu \nu \rho}=3 \partial_{[\mu} B_{\nu \rho]}-\frac{3}{2}\left(A_{[\mu}^{(1) m} F_{\nu \rho] m}^{(2)}+F_{[\mu \nu}^{(1) m} A_{\rho] m}^{(2)}\right) \\
H_{\mu \nu m}=F_{\mu \nu m}^{(2)}-B_{m n} F_{\mu \nu}^{(1) n} \\
H_{\mu m n}=\nabla_{\mu} B_{m n} \\
H_{m n p}=0
\end{array}\right.
$$

where we have defined the Abelian field strengths

$$
\left\{\begin{array}{l}
F_{\mu \nu}^{(1) m}=\partial_{\mu} A_{\nu}^{(1) m}-\partial_{\nu} A_{\mu}^{(1) m} \\
F_{\mu \nu m}^{(2)}=\partial_{\mu} A_{\nu m}^{(2)}-\partial_{\nu} A_{\mu m}^{(2)}
\end{array}\right.
$$

In terms of these objects, after dimensional reduction, the action (2.1) then takes the form [3]

\footnotetext{
${ }^{1}$ Note that it is not the procedure that is used on $\hat{B}$, as pointed out in Ref. [27].
}

$$
\begin{aligned}
I_{0}= & \int \mathrm{d}^{D} x \sqrt{-g} e^{-\Phi}\left(R+\partial_{\mu} \Phi \partial^{\mu} \Phi-\frac{1}{12} H_{\mu \nu \rho} H^{\mu \nu \rho}\right. \\
& +\frac{1}{4} \operatorname{Tr}\left(\partial_{\mu} G \partial^{\mu} G^{-1}\right)+\frac{1}{4} \operatorname{Tr}\left(G^{-1} \partial_{\mu} B G^{-1} \partial^{\mu} B\right) \\
& \left.-\frac{1}{4} F_{\mu \nu}^{(1) m} G_{m n} F^{(1) \mu \nu n}-\frac{1}{4} H_{\mu \nu m} G^{m n} H_{n}^{\mu \nu}\right),
\end{aligned}
$$

with the rescaled dilaton $\Phi=\hat{\phi}-\frac{1}{2} \log \left(\operatorname{det}\left(G_{m n}\right)\right)$. In this form, the action features an explicit GL $(d)$ symmetry, as guaranteed by toroidal reduction. The symmetry enhancement to $\mathrm{O}(d, d, \mathbb{R})$ can be made manifest upon regrouping the vector fields $A_{\mu}^{(1) m}$ and $A_{\mu m}^{(2)}$ into a single $\mathrm{O}(d, d, \mathbb{R})$ vector

$$
\mathcal{A}_{\mu}{ }^{M}=\left(\begin{array}{c}
A_{\mu}^{(1) m} \\
A_{\mu m}^{(2)}
\end{array}\right)
$$

and the scalar fields $G_{m n}, B_{m n}$ into an $\mathrm{O}(d, d, \mathbb{R})$ matrix $\mathcal{H}_{M N}$ as

$$
\mathcal{H}_{M N}=\left(\begin{array}{cc}
G_{m n}-B_{m p} G^{p q} B_{q n} & B_{m p} G^{p n} \\
-G^{m p} B_{p n} & G^{m n}
\end{array}\right) .
$$

Throughout, the fundamental $\mathrm{O}(d, d, \mathbb{R})$ indices are raised and lowered using the constant $\mathrm{O}(d, d, \mathbb{R})$ invariant matrix

$$
\eta^{M N}=\left(\begin{array}{cc}
0 & \delta^{m}{ }_{n} \\
\delta_{m}{ }^{n} & 0
\end{array}\right),
$$

so that $\mathcal{H}^{-1}$ is defined as $\mathcal{H}^{M N}=\eta^{M P} \mathcal{H}_{P Q} \eta^{Q N}$. In terms of the fields (2.8) and (2.9), the reduced action (2.7) may be cast into the manifestly $\mathrm{O}(d, d, \mathbb{R})$ invariant form [3]

$$
\begin{aligned}
I_{0}= & \int \mathrm{d}^{D} x \sqrt{-g} e^{-\Phi}\left(R+\partial_{\mu} \Phi \partial^{\mu} \Phi+\frac{1}{8} \partial_{\mu} \mathcal{H}_{M N} \partial^{\mu} \mathcal{H}^{M N}\right. \\
& \left.-\frac{1}{4} \mathcal{F}_{\mu \nu}{ }^{M} \mathcal{H}_{M N} \mathcal{F}^{\mu \nu N}-\frac{1}{12} H_{\mu \nu \rho} H^{\mu \nu \rho}\right)
\end{aligned}
$$

where $\mathcal{F}_{\mu \nu}{ }^{M}=2 \partial_{[\mu} \mathcal{A}_{\nu]}{ }^{M}$ is the Abelian field-strength associated with the vectors (2.8). In terms of the covariant objects (2.8) and (2.9), the infinitesimal $\mathrm{O}(d, d, \mathbb{R})$ variations of the fields are given by

$\left\{\begin{array}{l}\delta_{\Gamma} g_{\mu \nu}=0, \\ \delta_{\Gamma} B_{\mu \nu}=0,\end{array} \quad\left\{\begin{array}{l}\delta_{\Gamma} \mathcal{H}_{M N}=\Gamma_{M}{ }^{P} \mathcal{H}_{P N}+\Gamma_{N}{ }^{P} \mathcal{H}_{M P}, \\ \delta_{\Gamma} \mathcal{F}_{\mu \nu}{ }^{M}=-\mathcal{F}_{\mu \nu}{ }^{N} \Gamma_{N}{ }^{M},\end{array}\right.\right.$

for $\Gamma_{M}{ }^{N} \in \mathfrak{p}(d, d, \mathbb{R})$. The action (2.11) is manifestly invariant under these transformations. For later convenience, we also rewrite the action in terms of the matrix $\mathcal{S}_{M}{ }^{N}=\mathcal{H}_{M P} \eta^{P N}$, 


$$
\begin{aligned}
I_{0}= & \int \mathrm{d}^{D} x \sqrt{-g} e^{-\Phi}\left(R+\partial_{\mu} \Phi \partial^{\mu} \Phi+\frac{1}{8} \operatorname{Tr}\left(\partial_{\mu} \mathcal{S} \partial^{\mu} \mathcal{S}\right)\right. \\
& \left.-\frac{1}{4} \mathcal{F}_{\mu \nu}{ }^{M} \mathcal{S}_{M}{ }^{N} \mathcal{F}^{\mu \nu}{ }_{N}-\frac{1}{12} H_{\mu \nu \rho} H^{\mu \nu \rho}\right) .
\end{aligned}
$$

Note that $\mathcal{S} \mathcal{S}=\mathbb{1}$, so that $\mathcal{S}$ is a constrained field.

\section{B. GL $(d)$ fields redefinitions}

Our aim is an extension of the previous construction to higher orders in $\alpha^{\prime}$. As usual, the study of higher-derivative terms requires one to carefully handle the ambiguities due to the possible nonlinear field redefinitions. In particular, the symmetry enhancement to $\mathrm{O}(d, d, \mathbb{R})$ will be possible only after identification of the proper field redefinitions. In this section, we describe the systematics of higher-order field redefinitions based on the two-derivative action (2.11), inspired by Refs. [10,11].

We consider the $\alpha^{\prime}$ extension of Eq. (2.11) as a perturbation series

$$
I=I_{0}+I_{1}+\mathcal{O}\left(\alpha^{2}\right)
$$

with the first-order term $I_{1} \sim \mathcal{O}\left(\alpha^{\prime}\right)$. In order to organize the possible ambiguities in $I_{1}$, we consider field redefinitions of the form

$$
\varphi \rightarrow \varphi+\alpha^{\prime} \delta \varphi
$$

where $\varphi$ denotes a generic field. Under such redefinitions of its fields, the variation of $I$ to order $\alpha^{\prime}$ arises exclusively from the variation of $I_{0}$ and takes the form

$$
\begin{aligned}
\delta I_{0}= & \alpha^{\prime} \int \mathrm{d}^{D} x \sqrt{-g} e^{-\Phi}\left[\mathcal{E}_{\Phi} \delta \Phi+\left(\mathcal{E}_{g}\right)_{\mu \nu} \delta g^{\mu \nu}\right. \\
& +\left(\mathcal{E}_{B}\right)_{\mu \nu} \delta B^{\mu \nu}+\left(\mathcal{E}_{G}\right)_{m n} \delta G^{m n}+\left(\mathcal{E}_{B}\right)^{m n} \delta B_{m n} \\
& \left.+\left(\mathcal{E}_{A^{(1)}}\right)_{m}^{\mu} \delta A_{\mu}^{(1) m}+\left(\mathcal{E}_{A^{(2)}}\right)^{\mu m} \delta A_{\mu m}^{(2)}\right]
\end{aligned}
$$

proportional to the field equations associated with the twoderivative action $I_{0}$

$$
\begin{aligned}
& \mathcal{E}_{\Phi}=-2 \square \Phi-R+\nabla_{\mu} \Phi \nabla^{\mu} \Phi+\frac{1}{12} H^{2}-\frac{1}{8} \operatorname{Tr}\left(\nabla_{\mu} \mathcal{S} \nabla^{\mu} \mathcal{S}\right)+\frac{1}{4} \mathcal{F}_{\mu \nu}{ }^{M} \mathcal{S}_{M}{ }^{N} \mathcal{F}^{\mu \nu}{ }_{N}, \\
& \left(\mathcal{E}_{g}\right)_{\mu \nu}=R_{\mu \nu}+\nabla_{\mu} \nabla_{\nu} \Phi-\frac{1}{4} H_{\mu \nu}^{2}+\frac{1}{8} \operatorname{Tr}\left(\nabla_{\mu} \mathcal{S} \nabla_{\nu} \mathcal{S}\right)-\frac{1}{2} \mathcal{F}_{\mu \rho}^{M} \mathcal{S}_{M}{ }^{N} \mathcal{F}_{\nu}{ }{ }_{N}+\frac{1}{2} g_{\mu \nu} \mathcal{E}_{\Phi}, \\
& \left(\mathcal{E}_{B}\right)_{\mu \nu}=\frac{1}{2}\left(\nabla^{\rho} H_{\rho \mu \nu}-\nabla^{\rho} \Phi H_{\rho \mu \nu}\right), \\
& \left(\mathcal{E}_{G}\right)_{m n}=\frac{1}{2}\left[-\square G_{m n}+\nabla_{\mu} \Phi \nabla^{\mu} G_{m n}-\left(\nabla_{\mu} G \nabla^{\mu} G^{-1} G\right)_{m n}+\left(\nabla_{\mu} B G^{-1} \nabla^{\mu} B\right)_{m n}\right. \\
& \left.+\frac{1}{2} G_{m p} F_{\mu \nu}^{(1) p} F^{(1) \mu \nu q} G_{q n}-\frac{1}{2} H_{\mu \nu m} H_{n}^{\mu \nu}\right], \\
& \left(\mathcal{E}_{B}\right)^{m n}=\frac{1}{2}\left[\left(G^{-1} \square B G^{-1}\right)^{m n}-\nabla_{\mu} \Phi\left(G^{-1} \nabla^{\mu} B G^{-1}\right)^{m n}+\left(G^{-1} \nabla_{\mu} B \nabla^{\mu} G^{-1}\right)^{m n}\right. \\
& \left.+\left(\nabla_{\mu} G^{-1} \nabla^{\mu} B G^{-1}\right)^{m n}+\frac{1}{2} G^{m p} H_{\mu \nu p} F^{(1) \mu \nu n}-\frac{1}{2} F^{(1) \mu \nu m} G^{n p} H_{\mu \nu p}\right], \\
& \left(\mathcal{E}_{A^{(1)}}\right)_{n}^{\nu}=\nabla_{\mu} F^{(1) \mu \nu m} G_{m n}-\nabla_{\mu} \Phi F^{(1) \mu \nu m} G_{m n}-\frac{1}{2} H^{\mu \nu \rho} H_{\mu \rho n}-\left(\mathcal{E}_{A^{(2)}}\right)^{\nu m} B_{m n} \\
& +F^{(1) \mu \nu m} \nabla_{\mu} G_{m n}-H^{\mu \nu}{ }_{m}\left(G^{-1} \nabla_{\mu} B\right)^{m}{ }_{n}+\left(\mathcal{E}_{B}\right)^{\mu \nu}\left(A_{\mu n}^{(2)}-B_{n m} A_{\mu}^{(1) m}\right), \\
& \left(\mathcal{E}_{A^{(2)}}\right)^{\nu m}=\nabla_{\mu} H^{\mu \nu}{ }_{n} G^{n m}-\nabla_{\mu} \Phi H^{\mu \nu}{ }_{n} G^{n m}+H^{\mu \nu}{ }_{n} \nabla_{\mu} G^{n m}+\frac{1}{2} H^{\mu \rho \nu} F_{\mu \rho}^{(1) m}+\left(\mathcal{E}_{B}\right)_{\mu \nu} A_{\mu}^{(1) m} \text {. }
\end{aligned}
$$

Here, $H_{\mu \nu}^{2}=H_{\mu \rho \sigma} H_{\nu}{ }^{\rho \sigma}, \nabla_{\mu}$ denotes the covariant derivative with respect to $g_{\mu \nu}$, and accordingly $\square=\nabla_{\mu} \nabla^{\mu}$. At order $\alpha^{\prime}$, the action thus is unique up to contributions proportional to the lowest-order field equations. In the next section, we will show that by field redefinitions (2.15), the transformation (2.16) together with partial integrations allows one to map all terms at order $\alpha^{\prime}$ to a basis which carries only first derivatives of all fields (except for the two-derivative terms within the Riemann tensor).

As an example, let us show how a term carrying the factor $\square \Phi$ can be replaced by terms carrying only products of first derivatives. Consider a generic term of $I_{1}$ of the form 


$$
Z=\alpha^{\prime} \int \mathrm{d}^{D} x \sqrt{-g} e^{-\Phi} X \square \Phi
$$

where $X$ is a function of $\Phi, R_{\mu \nu \rho \sigma}, H_{\mu \nu \rho}, G_{m n}, B_{m n}, F_{\mu \nu}^{(1) m}$, and $H_{\mu \nu m}$ (and their derivatives) which carries exactly two derivatives. Redefining the dilaton and the metric as Eq. (2.15) with $\delta g_{\mu \nu}=\lambda g_{\mu \nu}$, Eq. (2.16) yields the transformation

$$
\begin{aligned}
\delta I_{0}= & \alpha^{\prime} \int \mathrm{d}^{D} x \sqrt{-g} e^{-\Phi}\left[\square \Phi(-2 \delta \Phi+\lambda(D+1))+\frac{1}{2} R(-2 \delta \Phi+\lambda(D+2))+\nabla_{\mu} \Phi \nabla^{\mu} \Phi\left(\delta \Phi-\frac{D}{2} \lambda\right)\right. \\
& \left.+\frac{1}{24} H^{2}(2 \delta \Phi-\lambda(D+6))-\frac{1}{16} \operatorname{Tr}\left(\nabla_{\mu} \mathcal{S} \nabla^{\mu} \mathcal{S}\right)(-2 \delta \Phi+\lambda(D+2))+\frac{1}{8} \mathcal{F}_{\mu \nu}{ }^{M} \mathcal{S}_{M}{ }^{N} \mathcal{F}^{\mu \nu}{ }_{N}(2 \delta \Phi-\lambda(D+4))\right] .
\end{aligned}
$$

With the particular choice

$$
\left\{\begin{array}{l}
\delta \Phi=\frac{1}{2}(D+2) X, \\
\lambda=X,
\end{array}\right.
$$

the new terms (2.19) cancel the term $Z$ and replace it by

$$
Z^{\prime}=\alpha^{\prime} \int \mathrm{d}^{D} x \sqrt{-g} e^{-\Phi} X\left(\nabla_{\mu} \Phi \nabla^{\mu} \Phi-\frac{1}{6} H^{2}-\frac{1}{4} \mathcal{F}_{\mu \nu}{ }^{M} \mathcal{S}_{M}{ }^{N} \mathcal{F}^{\mu \nu}{ }_{N}\right)
$$

which carries only products of first-order derivatives. In the same fashion, all the four-derivative terms carrying the leading two-derivative contributions from the field equations (2.17) can be transformed into terms carrying only products of first-order derivatives. We may summarize the resulting replacement rules as

$$
\begin{aligned}
\square \Phi \rightarrow \mathcal{Q}_{\Phi}= & \nabla_{\mu} \Phi \nabla^{\mu} \Phi-\frac{1}{6} H^{2}-\frac{1}{4} \mathcal{F}_{\mu \nu}^{M} \mathcal{S}_{M}{ }^{N} \mathcal{F}_{N}^{\mu \nu}, \\
R_{\mu \nu} \rightarrow \mathcal{Q}_{g \mu \nu}= & -\nabla_{((\mu} \nabla_{\nu))} \Phi+\frac{1}{4} H_{\mu \nu}^{2}-\frac{1}{8} \operatorname{Tr}\left(\nabla_{\mu} \mathcal{S} \nabla_{\nu} \mathcal{S}\right)+\frac{1}{2} \mathcal{F}_{\mu \rho}^{M} \mathcal{S}_{M}{ }^{N} \mathcal{F}_{\nu}{ }_{N} \\
& -\frac{1}{D} g_{\mu \nu}\left(\nabla_{\rho} \Phi \nabla^{\rho} \Phi-\frac{1}{6} H^{2}-\frac{1}{4} \mathcal{F}_{\rho \sigma}^{M} \mathcal{S}_{M}{ }^{N} \mathcal{F}_{N}^{\rho \sigma}\right), \\
\nabla^{\mu} H_{\mu \rho \sigma} \rightarrow \mathcal{Q}_{B \rho \sigma}= & \nabla^{\mu} \Phi H_{\mu \rho \sigma}, \\
\square G_{m n} \rightarrow \mathcal{Q}_{G m n}= & \nabla_{\mu} \Phi \nabla^{\mu} G_{m n}-\nabla_{\mu} G_{m p} \nabla^{\mu} G^{p q} G_{q n}-\frac{1}{2} H_{\mu \nu m} H^{\mu \nu}{ }_{n}+\nabla_{\mu} B_{m p} G^{p q} \nabla^{\mu} B_{q n}+\frac{1}{2} F_{\mu \nu}^{(1) p} G_{p m} F^{(1) \mu \nu q} G_{q n}, \\
\square B_{m n} \rightarrow \mathcal{Q}_{B m n}= & \nabla_{\mu} \Phi \nabla^{\mu} B_{m n}-\nabla_{\mu} B_{m p} \nabla^{\mu} G^{p q} G_{q n}-G_{m p} \nabla^{\mu} G^{p q} \nabla_{\mu} B_{q n}-\frac{1}{2} H_{\mu \nu m} F^{(1) \mu \nu p} G_{p n}+\frac{1}{2} G_{m p} F^{(1) \mu \nu p} H_{\mu \nu n}, \\
\nabla_{\mu} F^{(1) \mu \nu m} \rightarrow \mathcal{Q}_{A^{(1)}}{ }^{\nu m}= & \nabla_{\mu} \Phi F^{(1) \mu \nu m}+H^{\mu \nu}{ }_{n} G^{n p} \nabla_{\mu} B_{p q} G^{q m}+\frac{1}{2} H^{\mu \nu \rho} H_{\mu \rho n} G^{n m}-F^{(1) \mu \nu n} \nabla_{\mu} G_{n p} G^{p m}, \\
\nabla_{\mu} H^{\mu \nu}{ }_{m} \rightarrow \mathcal{Q}_{A^{(2)}{ }_{m}{ }_{m}}= & \nabla_{\mu} \Phi H^{\mu \nu}{ }_{m}-H^{\mu \nu}{ }_{n} \nabla_{\mu} G^{n p} G_{p m}+\frac{1}{2} H^{\mu \nu \rho} F_{\mu \rho}^{(1) n} G_{n m} .
\end{aligned}
$$

Double parenthesis $((\cdots))$ in the second line refer to traceless symmetrization. The associated field redefinitions are collected in Table I. As we will show in Sec. IV, all other four-derivative terms can be mapped into the terms listed in Table I upon using partial integration and Bianchi identities.

\section{O $(d, d, \mathbb{R})$ INVARIANT BASIS AT ORDER $\alpha^{\prime}$}

In this section, we present the construction of an explicit $\mathrm{O}(d, d, \mathbb{R})$ invariant basis for the four-derivative terms in $D$ dimensions. We discuss the general counting of independent terms for building an action upon modding out field redefinitions and partial integrations. At order $\alpha^{\prime}$ we find that the number of independent terms is 61 and coincides with the number of terms that can be built from products of first-order derivatives (and the Riemann tensor). We confirm the number by an explicit construction of a 61-dimensional basis which we use subsequently in order to organize the result of the explicit torus reduction. 
TABLE I. Replacement rules for the terms carrying the leading two-derivative contribution from the field equations descending from the two-derivative action (2.7) and associated field redefinitions. The explicit replacement rules are given in Eq. (2.22).

\begin{tabular}{lll}
\hline \hline Term in the action & \multicolumn{1}{c}{ Field redefinitions } & Replacement \\
\hline$\alpha^{\prime} X \square \Phi$ & $\left\{\begin{array}{l}\delta \Phi=\frac{1}{2}(D+2) X \\
\delta g_{\mu \nu}=g_{\mu \nu} X\end{array}\right.$ & $\alpha^{\prime} X \mathcal{Q}_{\Phi}$ \\
$\alpha^{\prime} X^{\mu \nu} R_{\mu \nu}$ & $\left\{\begin{array}{l}\delta g_{\mu \nu}=-X_{(\mu \nu)}-\frac{1}{D} g_{\mu \nu} X_{\rho}{ }^{\rho} \\
\delta \Phi=-\frac{1}{D} X_{\mu}{ }^{\mu}\end{array}\right.$ & $\alpha^{\prime} X^{\mu \nu} \mathcal{Q}_{g \mu \nu}$ \\
$\alpha^{\prime} X^{\mu \nu} \nabla^{\rho} H_{\rho \mu \nu}$ & $\delta B_{\mu \nu}=-2 X_{[\mu \nu]}$ & $\alpha^{\prime} X^{\mu \nu} \mathcal{Q}_{B \mu \nu}$ \\
$\alpha^{\prime} X^{m n} \square G_{m n}$ & $\delta G^{m n}=2 X^{(m n)}$ & $\alpha^{\prime} X^{m n} \mathcal{Q}_{G m n}$ \\
$\alpha^{\prime} X^{m n} \square B_{m n}$ & $\delta B_{m n}=-2 G_{m p} X^{[p q]} G_{q n}$ & $\alpha^{\prime} X^{m n} \mathcal{Q}_{B m n}$ \\
$\alpha^{\prime} X_{\nu m} \nabla_{\mu} F^{(1) \mu \nu m}$ & $\left\{\begin{array}{l}\delta A_{\mu}^{(1) m}=-X_{\mu n} G^{n m} \\
\delta A_{\mu m}^{(2)}=-X_{\mu n} G^{n p} B_{m p} \\
\delta B_{\mu \nu}=\left(A_{[\mu \mid m}^{(2)} X_{\mid \nu] n} G^{m n}-B_{m n} A_{[\mu}^{(1) n} X_{\nu] p} G^{m p}\right)\end{array}\right.$ \\
$\alpha^{\prime} X_{\nu}{ }^{m} \nabla_{\mu} H^{\mu \nu}{ }_{m}$ & $\left\{\begin{array}{l}\delta A_{\mu m}^{(2)}=-X_{\mu}{ }^{n} G_{n m} \\
\delta B_{\mu \nu}=A_{[\mu}^{(1) m} X_{\nu]}{ }^{n} G_{m n}\end{array}\right.$ & $\alpha^{\prime} X_{\nu m} \mathcal{Q}_{A^{(1)}}{ }^{\nu m}$
\end{tabular}

\section{A. Counting independent terms}

Following the general discussion of field redefinition ambiguities of the last section, we first count the number of independent terms modulo the two-derivative field equations (2.22) and Bianchi identities. At this stage we do not yet restrict to Lorentz scalars; i.e., we keep all $D$-dimensional spacetime indices uncontracted. In a second step we will restrict to Lorentz scalars and account for the freedom of partial integration. We start by defining the alphabet whose letters are the $\mathrm{O}(d, d, \mathbb{R})$ invariant building blocks in the various matter sectors (dilaton, scalars, vectors, 2-forms, metric) before identifying all possible symmetric words in these letters. We only count manifestly $\mathrm{O}(d, d, \mathbb{R})$ and gauge invariant terms, i.e., neglect possible Chern-Simons and topological terms which we will have to treat separately.

\section{Dilaton}

The independent building blocks carrying the dilaton are given by powers of derivatives

$$
\mathcal{B}_{\text {dil }}=\left\{\nabla_{\left(\left(\mu_{1}\right.\right.} \cdots \nabla_{\left.\left.\mu_{n}\right)\right)} \Phi \mid n \in \mathbb{N}^{*},\left\{\mu_{1}, \ldots, \mu_{n}\right\}\right\}
$$

with the double parentheses $((\cdots))$ indicating traceless symmetrization in order to divide out field equations. We may encode the set of letters (3.1) into a partition function

$$
\mathcal{Z}_{\mathrm{dil}}=u\left(\frac{1-q^{2}}{(1-q)^{\mathbf{v}_{D}}}-1\right)
$$

such that upon expanding (3.2) into a series in $q$ every term represents a letter with exponents counting the number of derivatives. We have also added a factor $u$ to keep track of the dilaton power when combining (3.2) with the other building blocks of the theory. We use the notation

$$
\begin{aligned}
& (1-q)^{\mathbf{v}_{D}}=1-q \mathbf{v}_{D}+q^{2}\left(\mathbf{v}_{D} \otimes \mathbf{v}_{D}\right)_{\text {alt }}-\cdots, \\
& \frac{1}{(1-q)^{\mathbf{v}_{D}}}=1+q \mathbf{v}_{D}+q^{2}\left(\mathbf{v}_{D} \otimes \mathbf{v}_{D}\right)_{\mathrm{sym}}+\cdots,
\end{aligned}
$$

with the $\mathrm{SO}(D)$ vectorial representation $\mathbf{v}_{D}$, in order to describe the tower of traceless symmetrized vectors. $\left(\mathbf{v}_{D} \otimes \mathbf{v}_{D}\right)_{\text {alt }}$ is the antisymmetric tensor product of two $\mathrm{SO}(D)$ vectors.

\section{Coset scalars}

The scalar fields parametrize the $\mathrm{SO}(d, d) /(\mathrm{SO}(d) \times$ $\mathrm{SO}(d))$ matrix $\mathcal{H}_{M N}$. In order to directly implement all constraints deriving from the coset structure, it is convenient to turn to the vielbeins

$$
\mathcal{H}_{M N}=E_{M}{ }^{A} \delta_{A B} E_{N}{ }^{B} \Rightarrow \partial_{\mu} \mathcal{H} \mathcal{H}^{-1}=2 E P_{\mu} E^{-1},
$$


with the coset currents defined by

$$
E^{-1} \partial_{\mu} E=Q_{\mu}+P_{\mu} \in \mathfrak{f} \oplus \mathfrak{p}=\mathfrak{g} \mathfrak{o}(d, d),
$$

where $\mathfrak{f}=\mathfrak{g} \mathfrak{o}(d) \oplus \mathfrak{s} \mathfrak{o}(d)$ and $\mathfrak{p}$ is its (noncompact) orthogonal complement. In terms of the currents $Q_{\mu}$ and $P_{\mu}$, global $\mathrm{SO}(d, d)$ invariance is ensured, and the counting problem reduces to identifying combinations that are invariant under local $\mathrm{SO}(d) \times \mathrm{SO}(d)$ transformations, i.e., built from $P_{\mu}$ 's and covariant derivatives $D_{\mu}=\partial_{\mu}+\operatorname{ad}_{Q_{\mu}}$. Moreover, we have integrability conditions

$$
\left[D_{\mu}, D_{\nu}\right]=Q_{\mu \nu} \propto\left[P_{\mu}, P_{\nu}\right], \quad D_{[\mu} P_{\nu]}=0,
$$

and field equations with leading second-order term $D^{\mu} P_{\mu}$ which implies that a basis of on-shell independent combinations is given by

$\left.\mathcal{B}_{P}=\left\{\nabla_{\left(\left(\mu_{1}\right.\right.} \cdots \nabla_{\mu_{n}} P_{\left.\mu_{n+1}\right)}\right) \in \mathfrak{p} \mid n \in \mathbb{N},\left\{\mu_{1}, \ldots, \mu_{n+1}\right\}\right\}$,

counted by the partition function

$$
\mathcal{Z}_{P}=p\left(\frac{1-q^{2}}{(1-q)^{\mathbf{v}_{D}}}-1\right)
$$

with the charge $p$ introduced to count the power of $P_{\mu}$ 's. It remains to count the independent $\mathrm{SO}(d) \times \mathrm{SO}(d)$ invariant single-trace combinations in the letters (3.7). With $P_{\mu}$ transforming in the $(\boldsymbol{d}, \boldsymbol{d})$ representation of $\mathrm{SO}(d) \times \mathrm{SO}(d)$, this amounts to counting ordered monomials and dividing out transpositions and cyclic shifts of even length. ${ }^{2}$ The result then follows from Polya's counting theorem [28] as

$$
\mathcal{Z}_{\text {sing.trace }}=-\frac{1}{2} \sum_{n} \frac{\varphi(n)}{n} \log \left(1-\mathcal{Z}_{\mathcal{P}, n}^{2}\right)+\frac{\mathcal{Z}_{\mathcal{P}, 2}}{2\left(1-\mathcal{Z}_{\mathcal{P}, 2}\right)},
$$

with Euler's totient function $\varphi(n)$ and $\mathcal{Z}_{\mathcal{P}, n}=\mathcal{Z}_{\mathcal{P}}\left(p^{n}, q^{n}\right)$.

\section{Vectors}

The (manifestly) gauge invariant building blocks in terms of the vector field are obtained by derivatives of its field strength subtracting Bianchi identities and contractions proportional to the field equations

$$
\mathcal{B}_{\mathcal{F}}=\left\{\nabla_{\left(\left(\mu_{1}\right.\right.} \cdots \nabla_{\left.\left.\mu_{n}\right)\right)} \mathcal{F}_{\nu_{1} \nu_{2}}{ }^{M}-\text { traces \& Bianchi } \mid n \in \mathbb{N}\right\}
$$

\footnotetext{
${ }^{2}$ In this counting, we neglect all the identities induced by the finite size $(2 d)$ of the $\mathrm{SO}(d, d)$ matrices; i.e., formally we count for $d=\infty$.
}

counted by the partition function (see, e.g., Ref. [29])

$$
\begin{aligned}
\mathcal{Z}_{\mathcal{F}} & =\sum_{n=0}^{\infty}\left(\begin{array}{ll|l}
\nu_{1} \mu_{1} \mid & \mu_{n} \\
\hline \nu_{2} & \text { traces }
\end{array}\right) \\
& =f\left(\frac{1}{q}-\frac{1-\mathbf{v}_{D} q\left(1-q^{2}\right)-q^{4}}{q(1-q)^{\mathbf{v}_{D}}}\right),
\end{aligned}
$$

where $f$ is a charge for the powers of $\mathcal{F}_{\mu \nu}{ }^{M}$. However, the letters (3.10) are not $\mathrm{O}(d, d, \mathbb{R})$ singlets but rather carry a fundamental vector index. $\mathrm{O}(d, d, \mathbb{R})$ invariant combinations are built from bilinears of Eq. (3.10) with the two $\mathrm{O}(d, d, \mathbb{R})$ vector indices contracted by products of the $\mathrm{O}(d, d, \mathbb{R})$ invariant $\eta_{M N}$, the scalar matrix $\mathcal{H}_{M N}$, and its derivatives. This is most conveniently counted by using the vielbeins (3.4) to convert the $\mathrm{O}(d, d, \mathbb{R})$ indices of Eq. (3.10) into $\mathrm{SO}(d) \times \mathrm{SO}(d)$ indices, such that the flattened field strength $\mathcal{F}_{\mu \nu}{ }^{M} E_{M}{ }^{A}$ decomposes into $(\boldsymbol{d}, \mathbf{1}) \oplus(\mathbf{1}, \boldsymbol{d})$ contributions which we denote by $\mathcal{F}_{L}$ and $\mathcal{F}_{R}$, respectively. The flattened letters (3.10) are then contracted out by arbitrary chains of letters from Eq. (3.7). This gives rise to three different types of terms:

$$
\begin{aligned}
& \left(\nabla \cdots \nabla \mathcal{F}_{L}\right)(\text { even chain of } \nabla \cdots \nabla P)\left(\nabla \cdots \nabla \mathcal{F}_{L}\right), \\
& \left(\nabla \cdots \nabla \mathcal{F}_{R}\right)(\text { even chain of } \nabla \cdots \nabla P)\left(\nabla \cdots \nabla \mathcal{F}_{R}\right), \\
& \left(\nabla \cdots \nabla \mathcal{F}_{L}\right)(\text { odd chain of } \nabla \cdots \nabla P)\left(\nabla \cdots \nabla \mathcal{F}_{R}\right) .
\end{aligned}
$$

Upon taking into account the reflection symmetries of the first two chains, the counting of $\mathrm{O}(d, d, \mathbb{R})$ invariant building blocks in the vector sector yields

$$
\begin{aligned}
\mathcal{Z}_{\mathcal{F F}}= & \frac{1}{2}\left(\frac{\mathcal{Z}_{\mathcal{F}, 2}}{1-\mathcal{Z}_{\mathcal{P}, 2}}+\frac{\mathcal{Z}_{\mathcal{F}}^{2}}{1-\mathcal{Z}_{\mathcal{P}}^{2}}\right)+\frac{1}{2}\left(\frac{\mathcal{Z}_{\mathcal{F}, 2}}{1-\mathcal{Z}_{\mathcal{P}, 2}}+\frac{\mathcal{Z}_{\mathcal{F}}^{2}}{1-\mathcal{Z}_{\mathcal{P}}^{2}}\right) \\
& +\mathcal{Z}_{\mathcal{F}} \frac{\mathcal{Z}_{\mathcal{P}}}{1-\mathcal{Z}_{\mathcal{P}}^{2}} \mathcal{Z}_{\mathcal{F}} \\
= & \frac{\mathcal{Z}_{\mathcal{F}}^{2}}{1-\mathcal{Z}_{\mathcal{P}}}+\frac{\mathcal{Z}_{\mathcal{F}, 2}}{1-\mathcal{Z}_{\mathcal{P}, 2}} .
\end{aligned}
$$

\section{Two-form}

Similarly, the independent (manifestly gauge invariant) building blocks carrying the 2-form $B_{\mu \nu}$ are counted by powers of derivatives on the field strength $H_{\mu \nu \rho}$ upon subtracting Bianchi identities and contractions proportional to the field equations

$\mathcal{B}_{H}=\left\{\nabla_{\left(\left(\mu_{1}\right.\right.} \cdots \nabla_{\left.\left.\mu_{n}\right)\right)} H^{\nu_{1} \nu_{2} \nu_{3}}-\right.$ traces \& Bianchi $\left.\mid n \in \mathbb{N}\right\}$,

giving rise to a partition function 


$$
\begin{aligned}
& \mathcal{Z}_{H}=\sum_{n=0}^{\infty}\left(\begin{array}{l|l|l|}
\hline \frac{\nu_{1}}{\nu_{2}}\left|\mu_{1}\right| & \mu_{n} \\
\hline \frac{\nu_{2}}{\nu_{3}} & \text { traces }
\end{array}\right) \\
& =h\left(\frac{1-q^{6}-q\left(1-q^{4}\right) \mathbf{v}_{D}+q^{2}\left(1-q^{2}\right)\left(\mathbf{v}_{D} \otimes \mathbf{v}_{D}\right)_{\text {alt }}}{q^{2}(1-q)^{\mathbf{v}_{D}}}\right. \\
& \left.-\frac{1}{q^{2}}\right)
\end{aligned}
$$

where $h$ is a charge for the powers of $H_{\mu \nu \rho}$.

\section{Metric}

For the external metric $g_{\mu \nu}$, we count derivatives of its Weyl tensor $C_{\nu_{1} \nu_{2} \nu_{3} \nu_{4}}$, subtracting traces and Bianchi identities, giving rise to the letters

$\mathcal{B}_{C}=\left\{\nabla_{\left(\left(\mu_{1}\right.\right.} \cdots \nabla_{\left.\left.\mu_{n}\right)\right)} C_{\nu_{1} \nu_{2} \nu_{3} \nu_{4}}-\right.$ traces \& Bianchi $\left.\mid n \in \mathbb{N}\right\}$,

which are counted as

$$
\begin{aligned}
\mathcal{Z}_{C}= & \sum_{n=0}^{\infty}\left(\begin{array}{l|l|l|}
\left|\nu_{1}\right| \nu_{2}\left|\mu_{1}\right| & \mu_{n} \\
\hline \nu_{3}\left|\nu_{4}\right| & \text { traces }
\end{array}\right) \\
= & c\left(\frac{q\left(1-q^{2}\right)\left(\mathbf{v}_{D} \otimes \mathbf{v}_{D}\right)_{\mathrm{sym}}-\left(1-q^{4}\right) \mathbf{v}_{D}}{q(1-q)^{\mathbf{v}_{D}}}\right. \\
& \left.+\left(\mathbf{v}_{D} \otimes \mathbf{v}_{D}\right)_{\text {alt }}+\frac{1}{q} \mathbf{v}_{D}\right),
\end{aligned}
$$

where $c$ is a charge for the powers of the Weyl tensor (or equivalently, the Riemann tensor).

\section{B. Spacetime singlets and partial integration}

Putting everything together, we have identified the manifestly $\mathrm{O}(d, d, \mathbb{R})$ and gauge invariant building blocks in the various sectors,

$$
\mathcal{Z}_{0}=\mathcal{Z}_{\text {dil }}+\mathcal{Z}_{H}+\mathcal{Z}_{C}+\mathcal{Z}_{\text {sing.trace }}+\mathcal{Z}_{\mathcal{F} F},
$$

with the different terms defined in Eqs. (3.2), (3.15), (3.17), (3.9), and (3.13), respectively. From these objects, we can construct the most general $\mathrm{O}(d, d, \mathbb{R})$ and gauge invariant terms as arbitrary polynomials in the letters of Eq. (3.18), counted as

$$
\mathcal{Z}_{\text {inv }}=\exp \left[\sum_{k} \frac{1}{k} \mathcal{Z}_{0, k}\right]
$$

So far, we have been counting combinations in all possible $\mathrm{SO}(D)$ representations, without restricting to $\mathrm{SO}(D)$ Lorentz scalars. In order to count the independent spacetime actions, we first project $\mathcal{Z}_{\text {inv }}$ to Lorentz scalars. Next, in order to subtract the ambiguities from partial integrations, we extract from $\mathcal{Z}_{\text {inv }}$ all possible $\mathrm{SO}(D)$ vectors $\mathcal{J}_{\mu}$ each of which gives rise to an ambiguity $\mathrm{d} * \mathcal{J}$ of the spacetime Lagrangian. On the other hand, currents with (off-shell) vanishing divergence $\mathrm{d} * \mathcal{J}=0$ do not define ambiguities; these are of the form $\mathcal{J}=* \mathrm{~d} * \mathcal{J}_{2}$ for a 2 -form $\mathcal{J}_{2}$, unless $* \mathcal{J}_{2}$ is of a vanishing divergence thus defined by a 3 -form $\mathcal{J}_{3}$, etc. To summarize, a basis of independent spacetime Lagrangians, after dividing out the freedom of partial integrations, is given by

$$
\mathcal{Z}_{\text {Lag }}=\left.\mathcal{Z}_{\text {inv }}(1-u q)^{\mathbf{v}_{D}}\right|_{\mathrm{SO}(D) \text { singlets }},
$$

in the notation of Eq. (3.3). ${ }^{3}$

\section{Some examples}

\section{Evaluation in $D=10$}

As a first test of the counting formula (3.20), we may evaluate it to order $\alpha^{\prime}$ in $D=10$ dimensions, i.e., for $d=0$, upon truncating out the vector and scalar sectors which do not exist at $d=0$. Then, in Eq. (3.18) only the contributions from metric, 2-form, and dilaton are taken into account. Evaluating Eq. (3.20) gives rise to the following types of terms at the four-derivative order

$$
\left\{R^{2}[1], \nabla^{2} H^{2}[1], R H^{2}[1], H^{4}[3], H^{2} \nabla^{2} \Phi[1], \nabla^{2} \Phi \nabla^{2} \Phi[1]\right\},
$$

where the multiplicities $[n]$ indicate the number of independent terms of the same type. This precisely reproduces the counting from Ref. [30] [cf. their Eq. (2.36)]. Let us recall that our counting only includes manifestly gauge invariant terms, so it does not account for the possible tendimensional gravitational Chern-Simons couplings.

\section{Evaluation in $D=1$}

Upon reduction to only one dimension, we can evaluate the counting formulas to all orders in closed form. In particular $\mathcal{Z}_{H}=\mathcal{Z}_{\mathcal{F}}=0$, while

$\mathcal{Z}_{\text {dil }}=u q, \quad \mathcal{Z}_{P}=p q, \quad \mathcal{Z}_{\text {sing.trace }}=\frac{p^{2} q^{2}}{1-p^{2} q^{2}}$,

and

$$
\mathcal{Z}_{C}=-c q^{2} \rightarrow-p^{2} q^{2}
$$

reflecting the fact that in $D=1$ the Einstein equations pose a constraint on the energy-momentum tensor. For Eqs. (3.18) and (3.19), we thus find

\footnotetext{
${ }^{3}$ Here, we have inserted a dilaton charge $u$, since all terms carry a global dilaton power $e^{-\Phi}$ such that partial integration brings in an extra dilaton derivative.
} 
$\mathcal{Z}_{0}=\frac{p^{4} q^{4}}{1-p^{2} q^{2}}+u q \Rightarrow \mathcal{Z}_{\text {inv }}=\prod_{n>1} \frac{1}{1-p^{2 n} q^{2 n}} \times \frac{1}{1-u q}$,

upon removing total derivatives (3.20) and thus

$$
\mathcal{Z}_{\mathrm{Lag}}=(1-q u) \mathcal{Z}_{\text {inv }}=\prod_{n>1} \frac{1}{1-p^{2 n} q^{2 n}},
$$

which precisely reproduces the counting from Ref. [11].

\section{Basis at order $\boldsymbol{\alpha}^{\prime}$}

Evaluating the counting formula (3.20) in generic dimension $D$ we infer that at order $\alpha^{\prime}$ there are 61 independent manifestly $\mathrm{O}(d, d, \mathbb{R})$ invariant four-derivative terms. While the general counting only determines the number of independent terms without selecting a particular basis, it turns out that at order $\alpha^{\prime}$ there is a distinguished explicit basis which is built from polynomials in terms carrying only first-order derivatives (and the Riemann tensor). Indeed, truncating the partition functions (3.2), (3.15), (3.17), (3.8), (3.11) to first order in derivatives, we may count from Eq. (3.19) the number of independent terms that carry first derivatives only, and find precisely 61 terms at order $\alpha^{\prime}{ }^{4}$

The basis at order $\alpha^{\prime}$ can thus be given in terms of polynomials in $R_{\mu \nu \rho}{ }^{\sigma}, H_{\mu \nu \rho}, \mathcal{F}_{\mu \nu}{ }^{M}, \nabla_{\mu} \mathcal{S}_{M}{ }^{N}$, and $\nabla_{\mu} \Phi$. Schematically, its elements take the form

$$
\begin{aligned}
& \left\{R^{2}[1], H^{4}[3],(\nabla \Phi)^{4}[1],(\nabla \mathcal{S})^{4}[5], \mathcal{F}^{4}[12], R H^{2}[1], R \mathcal{F}^{2}[2], H^{2}(\nabla \Phi)^{2}[2], H^{2}(\nabla \mathcal{S})^{2}[2], H^{2} \mathcal{F}^{2}[8],(\nabla \Phi)^{2}(\nabla \mathcal{S})^{2}[2],\right. \\
& \left.\quad(\nabla \Phi)^{2} \mathcal{F}^{2}[4],(\nabla \mathcal{S})^{2} \mathcal{F}^{2}[10], H \nabla \Phi \mathcal{F}^{2}[2], H \nabla \mathcal{S} \mathcal{F}^{2}[3], \nabla \Phi \nabla \mathcal{S} \mathcal{F}^{2}[3]\right\} .
\end{aligned}
$$

We give the explicit expressions for all the basis elements in Appendix A. In the following we will exhibit the $\mathrm{O}(d, d$, $\mathbb{R})$ invariance of the dimensionally reduced action by expanding the reduced action in the basis (3.26).

\section{COMPACTIFICATION OF THE FOUR-DERIVATIVE ACTION}

The first-order $\alpha^{\prime}$ extension of the action of the bosonic string (2.1) has been known for some time [30] and is given up to field redefinitions by

$$
\hat{I}_{1}=\frac{1}{4} \alpha^{\prime} \int \mathrm{d}^{D+d} X \sqrt{-\hat{g}} e^{-\hat{\phi}}\left(\hat{R}_{\hat{\mu} \hat{\nu} \hat{\rho} \hat{\sigma}} \hat{R}^{\hat{\mu} \hat{\nu} \hat{\rho} \hat{\sigma}}-\frac{1}{2} \hat{H}^{\hat{\mu} \hat{\nu} \hat{\lambda}} \hat{H}^{\hat{\rho} \hat{\sigma}} \hat{\lambda}_{\hat{\lambda}} \hat{R}_{\hat{\mu} \hat{\nu} \hat{\rho} \hat{\sigma}}-\frac{1}{8} \hat{H}_{\hat{\mu} \hat{\nu}}^{2} \hat{H}^{2 \hat{\mu} \hat{\nu}}+\frac{1}{24} \hat{H}_{\hat{\mu} \hat{\nu} \hat{\rho}} \hat{H}_{\hat{\sigma}}^{\hat{\mu}} \hat{H}_{\hat{\lambda}^{\hat{\imath}}} \hat{H}^{\hat{\rho}}{ }_{\hat{\tau}}^{\hat{\sigma}}\right) .
$$

In this section, we compactify separately all of its terms on a $d$-torus, using the ansätze (2.3) and (2.4). We fix the freedom of partial integration and possible field redefinitions by converting all terms into polynomials of first-order derivatives (and the Riemann tensor). To do so, we systematically use partial integration and Bianchi identities to bring all terms carrying second-order derivatives into a form corresponding to the first column of Table I, which can then be converted to the desired form by means of field redefinitions as discussed in Sec. II B. In the next section, we then compare the result to the $\mathrm{O}(d, d, \mathbb{R})$ basis of Sec. III D.

The reduction of the 3-form field strength $\hat{H}_{\hat{\mu} \hat{\nu} \hat{\rho}}$ is given in Eq. (2.5). For the reduction of the Riemann tensor, we follow the results of Ref. [31] and give the lower-dimensional components in flat indices as

$$
\begin{aligned}
\hat{R}_{\alpha \beta \gamma \delta} & =R_{\alpha \beta \gamma \delta}-\frac{1}{2}\left[-G_{m n} F_{\alpha[\gamma}^{(1) m} F_{\delta] \beta}^{(1) n}+G_{m n} F_{\alpha \beta}^{(1) m} F_{\gamma \delta}^{(1) n}\right], \\
\hat{R}_{\alpha \beta \gamma d} & =\left[\nabla_{[\alpha} F_{\beta] \gamma}^{(1) p}-\frac{1}{2}\left(G_{m n} \nabla_{[\alpha} G^{n p} F_{\beta] \gamma}^{(1) m}-F_{\alpha \beta}^{(1) m} G_{m n} \nabla_{\gamma} G^{n p}\right)\right] E_{p d}, \\
\hat{R}_{\alpha \beta c d} & =\frac{1}{2}\left[F_{\alpha}^{(1) \gamma m} F_{\gamma \beta}^{(1) q}-\nabla_{\alpha} G^{m n} G_{n p} \nabla_{\beta} G^{p q}\right] E_{m[c} E_{|q| d]}, \\
\hat{R}_{\alpha b \gamma d} & =\frac{1}{4}\left[2 \nabla_{\alpha} \nabla_{\gamma} G^{m q}-2 \nabla_{\alpha} G^{m n} G_{n p} \nabla_{\gamma} G^{p q}-\nabla_{\gamma} G^{m n} G_{n p} \nabla_{\alpha} G^{p q}+F_{\gamma \varepsilon}^{(1) m} F_{\alpha}^{(1) \varepsilon q}\right] E_{m b} E_{q d}, \\
\hat{R}_{a b \gamma d} & =-\frac{1}{2} F_{\gamma \varepsilon}^{(1) m} \nabla^{\varepsilon} G^{n p} E_{m[a} E_{|n| b]} E_{p d}, \\
\hat{R}_{a b c d} & =-\frac{1}{2} \nabla_{\varepsilon} G^{m n} \nabla^{\varepsilon} G^{p q} E_{m a} E_{p b} E_{n[c} E_{|q| d]} .
\end{aligned}
$$

\footnotetext{
${ }^{4}$ At order $\alpha^{\prime 2}$ this pattern breaks down. The general counting (3.20) reveals 1817 independent terms at order $\alpha^{\prime 2}$, whereas there are only 1212 independent polynomials that can be constructed in terms of first-order derivatives. This general case differs from the situation encountered in the reduction to $D=1$ dimensions where one can always find a basis carrying no more than first-order time derivatives [10].
} 


\section{A. Reduction of the various terms}

We reduce the action (4.1) term by term.

\section{Reduction of $\hat{H}_{\hat{\mu} \hat{\nu}}^{2} \hat{H}^{2 \hat{\mu} \hat{\nu}}$}

Upon compactification, we obtain

$$
\begin{aligned}
\hat{H}_{\hat{\mu} \hat{\nu}}^{2} \hat{H}^{2 \hat{\mu} \hat{\nu}}= & H_{\mu \nu}^{2} H^{2 \mu \nu}+4 H_{\mu \nu}^{2} H_{\rho m}^{\mu} H^{\nu \rho m}-2 H^{2}{ }^{\mu \nu} H_{\mu \nu m} H_{\nu}{ }^{n m}+4 H_{\mu \nu m} H^{\mu \rho m} H^{\nu \sigma n} H_{\rho \sigma n} \\
& -4 H^{\mu \rho p} H^{\nu}{ }_{\rho p} H_{\mu m n} H_{\nu}{ }^{n m}+H_{\mu m n} H_{\nu}{ }^{m n} H^{\mu}{ }_{p q} H^{\nu q p}+2 H^{\mu \nu \lambda} H^{\rho \sigma}{ }_{\lambda} H_{\mu \nu m} H_{\rho \sigma}{ }^{m} \\
& +8 H^{\mu \nu \rho} H_{\mu \nu m} H_{\sigma}{ }^{m n} H^{\sigma}{ }_{\rho n}-8 H_{\mu \rho m} H^{\mu m n} H^{\nu}{ }_{n p} H_{\nu}{ }^{\rho p}+H_{\mu \nu m} H_{\rho \sigma}{ }^{m} H^{\mu \nu n} H^{\rho \sigma}{ }_{n} \\
& -4 H_{\mu \nu m} H_{\rho}{ }^{m n} H^{\rho}{ }_{n p} H^{\mu \nu p}+4 H_{\mu m n} H^{\nu n p} H_{\nu p q} H^{\mu q m}+4 H_{m n p} H_{\mu \nu \rho} H^{m n \rho} H^{\mu \nu p} \\
& +8 H_{m n p} H_{\mu \nu q} H^{\mu p q} H^{\nu m n}+2 H_{m n p} H_{\mu q r} H^{\mu m n} H^{p q r}+2 H_{m n p} H^{m n q} H_{\mu \nu}{ }^{p} H^{\mu \nu q} \\
& +4 H_{m n p} H^{m n q} H^{\mu p r} H_{\mu q r}+H_{m n r} H^{m n s} H_{p q s} H^{p q r} .
\end{aligned}
$$

Using Eq. (2.5), this takes the form

$$
\begin{aligned}
\hat{H}_{\hat{\mu} \hat{\nu}}^{2} \hat{H}^{2 \hat{\mu} \hat{\nu}}= & H_{\mu \nu}^{2} H^{2 \mu \nu}+4 \operatorname{Tr}\left(\nabla_{\mu} B G^{-1} \nabla^{\mu} B G^{-1} \nabla_{\nu} B G^{-1} \nabla^{\nu} B G^{-1}\right) \\
& +\operatorname{Tr}\left(\nabla_{\mu} B G^{-1} \nabla_{\nu} B G^{-1}\right) \operatorname{Tr}\left(\nabla^{\mu} B G^{-1} \nabla^{\nu} B G^{-1}\right)+H_{\mu \nu m} G^{m n} H_{\rho \sigma n} H^{\mu \nu}{ }_{p} G^{p q} H^{\rho \sigma}{ }_{q} \\
& +4 H_{\mu \nu m} G^{m n} H^{\mu \rho}{ }_{n} H_{\rho \sigma p} G^{p q} H^{\nu \sigma}{ }_{q}-2 H^{2 \mu} \operatorname{Tr}\left(\nabla_{\mu} B G^{-1} \nabla_{\nu} B G^{-1}\right) \\
& +4 H^{2 \mu \nu} H_{\mu \rho m} G^{m n} H_{\nu}{ }_{n}-4 \operatorname{Tr}\left(\nabla^{\mu} B G^{-1} \nabla^{\nu} B G^{-1}\right) H_{\mu \rho m} G^{m n} H_{\nu}{ }_{n}{ }{ }^{\mu \nu \lambda}{ }^{\rho \sigma} H_{\mu \nu m} G^{m n} H_{\rho \sigma n}-8 H_{\mu \rho m}\left(G^{-1} \nabla^{\mu} B G^{-1} \nabla_{\nu} B G^{-1}\right)^{m n} H^{\nu \rho}{ }_{n} \\
& +2 H^{\mu \nu \lambda} H_{\lambda}{ }^{\mu \nu}{ }_{n}, \\
& -8 H^{\mu \nu \rho} H_{\mu \nu m}\left(G^{-1} \nabla^{\sigma} B G^{-1}\right)^{m n} H_{\rho \sigma n}-4 H_{\mu \nu m}\left(G^{-1} \nabla_{\rho} B G^{-1} \nabla^{\rho} B G^{-1}\right)^{m n} H^{\mu}{ }_{n}
\end{aligned}
$$

where all terms carry first-order derivatives only, i.e., are already of the desired form.

\section{Reduction of $\hat{\boldsymbol{H}}_{\hat{\mu} \hat{\nu} \hat{\rho}} \hat{\boldsymbol{H}}_{\hat{\sigma}}^{\hat{\mu}} \hat{\boldsymbol{H}}_{\hat{\lambda}}^{\hat{\nu}} \hat{\boldsymbol{H}}_{\hat{\tau}}^{\hat{\rho}}{ }_{\hat{\boldsymbol{\sigma}}}$}

Upon compactification, we obtain

$$
\begin{aligned}
\hat{H}_{\hat{\mu} \hat{\nu} \hat{\rho}} \hat{H}_{\hat{\sigma}}^{\hat{\mu}}{ }_{\hat{\sigma}}^{\hat{\lambda}} \hat{H}^{\hat{\nu}}{ }_{\lambda}^{\hat{\imath}} \hat{H}^{\hat{\rho}}{ }_{\hat{\imath}}^{\hat{\sigma}}= & H_{\mu \nu \rho} H^{\mu}{ }_{\sigma}{ }^{\lambda} H^{\nu}{ }_{\lambda}{ }^{\tau} H^{\rho}{ }_{\tau}{ }^{\sigma}+6 H^{\mu \nu \lambda} H^{\rho \sigma}{ }_{\lambda} H_{\mu \rho m} H_{\nu \sigma}{ }^{m} \\
& -12 H^{\mu \nu \rho} H_{\mu \sigma m} H_{\nu}{ }^{m n} H_{\rho}{ }^{\sigma}{ }_{n}+4 H^{\mu \nu \rho} H_{\mu m n} H_{\nu}{ }^{n p} H_{\rho p}{ }^{m}+3 H_{\mu \nu m} H_{\rho \sigma}{ }^{m} H^{\mu \rho}{ }_{n} H^{\nu \sigma n} \\
& -12 H_{\mu \nu m} H_{\rho}{ }^{m n} H^{\nu}{ }_{n p} H^{\mu \rho p}+3 H_{\mu m n} H_{\nu}{ }^{n p} H^{\mu}{ }_{p q} H^{\nu p m}+4 H_{m n p} H^{m}{ }_{\mu \nu} H_{\rho}{ }^{\mu n} H^{\rho \nu p} \\
& +12 H_{m n p} H^{\mu m q} H^{\nu n}{ }_{q} H_{\mu \nu}{ }^{p}+6 H_{m n r} H_{p q}{ }^{r} H^{m p}{ }_{\mu} H^{n q \mu}+H_{m n p} H^{m}{ }_{q}{ }^{r} H^{n}{ }_{r}{ }^{s} H^{p}{ }_{s}{ }^{q} .
\end{aligned}
$$

Using Eq. (2.5), this takes the form

$$
\begin{aligned}
& \hat{H}_{\hat{\mu} \hat{\nu} \hat{\rho}} \hat{H}^{\hat{\mu}}{ }_{\hat{\sigma}}^{\hat{\lambda}} \hat{H}^{\hat{\nu}}{ }_{\hat{\lambda}}^{\hat{\imath}} \hat{H}^{\hat{\rho}}{ }_{\hat{\tau}}{ }^{\hat{\sigma}}=H_{\mu \nu \rho} H^{\mu}{ }_{\sigma}{ }^{\lambda} H^{\nu}{ }_{\lambda}{ }^{\tau} H^{\rho}{ }_{\tau}{ }^{\sigma}+3 H_{\mu \nu m} G^{m n} H_{\rho \sigma n} H^{\mu \rho}{ }_{p} G^{p q} H^{\nu \sigma}{ }_{q} \\
& +6 H^{\mu \nu \lambda} H^{\rho \sigma}{ }_{\lambda} H_{\mu \rho m} G^{m n} H_{\nu \sigma n}+3 \operatorname{Tr}\left(\nabla_{\mu} B G^{-1} \nabla_{\nu} B G^{-1} \nabla^{\mu} B G^{-1} \nabla^{\nu} B G^{-1}\right) \\
& -12 H^{\mu \nu \rho} H_{\mu \sigma m}\left(G^{-1} \nabla_{\nu} B G^{-1}\right)^{m n} H_{\rho}{ }^{\sigma}{ }_{n}-12 H_{\mu \nu m}\left(G^{-1} \nabla_{\rho} B G^{-1} \nabla^{\nu} B G^{-1}\right)^{m n} H^{\mu \rho}{ }_{n} \\
& +4 H^{\mu \nu \rho} \operatorname{Tr}\left(\nabla_{\mu} B G^{-1} \nabla_{\nu} B G^{-1} \nabla_{\rho} B G^{-1}\right),
\end{aligned}
$$

where again all terms carry first-order derivatives only, i.e., are already of the desired form. 


\section{Reduction of $\hat{\boldsymbol{R}}_{\hat{\mu} \hat{\nu} \hat{\rho} \hat{\sigma}} \hat{\boldsymbol{R}}^{\hat{\mu} \hat{\nu} \hat{\rho} \hat{\sigma}}$}

Splitting the $D+d$ indices $\hat{\mu}$ as $\hat{\mu} \rightarrow\{\mu, m\}$, we obtain

$$
\begin{aligned}
\hat{R}_{\hat{\mu} \hat{\nu} \hat{\rho} \hat{\sigma}} \hat{R}^{\hat{\mu} \hat{\nu} \hat{\rho} \hat{\sigma}}= & \hat{R}_{\mu \nu \rho \sigma} \hat{R}^{\mu \nu \rho \sigma}+4 \hat{R}_{\mu \nu \rho m} \hat{R}^{\mu \nu \rho m}+2 \hat{R}_{\mu \nu m n} \hat{R}^{\mu \nu m n} \\
& +4 \hat{R}_{\mu m \nu n} \hat{R}^{\mu m \nu n}+4 \hat{R}_{m n \mu p} \hat{R}^{m n \mu p}+\hat{R}_{m n p q} \hat{R}^{m n p q} .
\end{aligned}
$$

Upon using Eq. (4.2), the reduction of the first term of the action (4.1) then yields

$$
\begin{aligned}
& \frac{\alpha^{\prime}}{4} \int \mathrm{d}^{D+d} X \sqrt{-\hat{g}} e^{-\hat{\phi}} \hat{R}_{\hat{\mu} \hat{\nu} \hat{\rho} \hat{\sigma}} \hat{R}^{\hat{\mu} \hat{\nu} \hat{\rho} \hat{\sigma}} \\
& \rightarrow \frac{\alpha^{\prime}}{4} \int \mathrm{d}^{D} x \sqrt{-g} e^{-\Phi}\left[R_{\mu \nu \rho \sigma} R^{\mu \nu \rho \sigma}-\frac{3}{2} R^{\mu \nu \rho \sigma} F_{\mu \nu}^{(1) m} G_{m n} F_{\rho \sigma}^{(1) n}+\frac{3}{2} \operatorname{Tr}\left(\nabla_{\mu} G^{-1} \nabla^{\mu} G \nabla_{\nu} G^{-1} \nabla^{\nu} G\right)\right. \\
&+\frac{5}{8} \operatorname{Tr}\left(\nabla_{\mu} G^{-1} \nabla_{\nu} G \nabla^{\mu} G^{-1} \nabla^{\nu} G\right)+\frac{1}{8} \operatorname{Tr}\left(\nabla_{\mu} G^{-1} \nabla_{\nu} G\right) \operatorname{Tr}\left(\nabla^{\mu} G^{-1} \nabla^{\nu} G\right) \\
&+\frac{3}{8} F_{\mu \nu}^{(1) m} G_{m n} F_{\rho \sigma}^{(1) n} F^{(1) \mu \nu p} G_{p q} F^{(1) \rho \sigma q}+\frac{1}{8} F_{\mu \nu}^{(1) m} G_{m n} F_{\rho \sigma}^{(1) n} F^{(1) \mu \rho p} G_{p q} F^{(1) \nu \sigma q} \\
&+\frac{1}{2} F_{\mu \nu}^{(1) m} G_{m n} F^{(1) \mu \rho n} F_{\rho \sigma}^{(1) p} G_{p q} F^{(1) \nu \sigma q}-\frac{1}{2} \operatorname{Tr}\left(\nabla^{\mu} G^{-1} \nabla_{\nu} G\right) F_{\mu \rho}^{(1) m} G_{m n} F^{(1) \nu \rho n} \\
&-\frac{3}{2} F_{\mu \nu}^{(1) m}\left(\nabla_{\rho} G \nabla^{\rho} G^{-1} G\right)_{m n} F^{(1) \mu \nu n}+\frac{1}{2} F_{\mu \nu}^{(1) m}\left(\nabla_{\rho} G \nabla^{\mu} G^{-1} G\right)_{m n} F^{(1) \rho \nu n} \\
&+\operatorname{Tr}\left(\nabla_{\mu} \nabla_{\nu} G^{-1} G \nabla^{\mu} \nabla^{\nu} G^{-1} G\right)+3 \operatorname{Tr}\left(\nabla_{\mu} \nabla_{\nu} G^{-1} \nabla^{\mu} G \nabla^{\nu} G^{-1} G\right)-6 \nabla_{\rho} F_{\mu \nu}^{(1) m} \nabla^{\mu} G_{m n} F^{(1) \nu \rho n} \\
&\left.+F_{\mu \nu}^{(1) m}\left(G \nabla^{\mu} \nabla_{\rho} G^{-1} G\right)_{m n} F^{(1) \rho \nu n}-2 \nabla_{\rho} F_{\mu \nu}^{(1) m} G_{m n} \nabla^{\mu} F^{(1) \nu \rho n}\right] .
\end{aligned}
$$

Apart from the Riemann tensor, only the five last terms contain second-order derivatives. Using partial integration and Bianchi identities, it is possible to transform those terms so that all second-order derivatives appear as the leading twoderivative contribution from the field Eqs. (2.17), i.e., appear within the first column of Table I. Details are given in Appendix B. Specifically, the remaining second-order derivative terms combine into

$$
\begin{aligned}
& \frac{\alpha^{\prime}}{4} \int \mathrm{d}^{D} x \sqrt{-g} e^{-\Phi}\left[\operatorname{Tr}\left(\square G^{-1} G \square G^{-1} G\right)-2 \nabla_{\mu} \Phi \operatorname{Tr}\left(\square G^{-1} G \nabla^{\mu} G^{-1}\right)\right. \\
& \quad+2 \operatorname{Tr}\left(\square G^{-1} G \nabla_{\nu} G^{-1} \nabla^{\nu} G\right)+\frac{1}{2} \operatorname{Tr}\left(\square G G^{-1} \nabla_{\nu} G \nabla^{\nu} G^{-1}\right)-\frac{5}{4} F_{\mu \nu}^{(1) m} \square G_{m n} F^{(1) \mu \nu n} \\
& \quad+\left(R_{\mu \nu}+\nabla_{\mu} \nabla_{\nu} \Phi\right)\left(\operatorname{Tr}\left(\nabla^{\mu} G^{-1} \nabla^{\nu} G\right)-2 F_{\mu \rho}^{(1) m} G_{m n} F_{\nu}^{(1) \rho n}\right) \\
& \quad+2 \nabla^{\mu} F_{\mu \nu}^{(1) m} G_{m n}\left(\nabla_{\rho} F^{(1) \rho \nu n}-\nabla_{\rho} \Phi F^{(1) \rho \nu n}\right) \\
& \left.\quad+\left(-2 \nabla^{\mu} \Phi F_{\mu \nu}^{(1) m} G_{m n}+3 F_{\mu \nu}^{(1) m} \nabla^{\mu} G_{m n}\right) \nabla_{\rho} F^{(1) \rho \nu n}\right]
\end{aligned}
$$

and can be eliminated by field redefinitions according to the rules defined in Table I. The explicit induced field redefinitions are collected in Eq. (B4). The final result of the reduction (4.8) then takes the form

$$
\begin{aligned}
& \frac{\alpha^{\prime}}{4} \int \mathrm{d}^{D+d} X \sqrt{-\hat{g}} e^{-\hat{\phi}} \hat{R}_{\hat{\mu} \hat{\nu} \hat{\rho} \hat{\sigma}} \hat{R}^{\hat{\mu} \hat{\nu} \hat{\rho} \hat{\sigma}} \\
& \rightarrow \frac{\alpha^{\prime}}{4} \int \mathrm{d}^{D} x \sqrt{-g} e^{-\Phi}\left[R_{\mu \nu \rho \sigma} R^{\mu \nu \rho \sigma}-\frac{1}{2} R^{\mu \nu \rho \sigma} F_{\mu \nu}^{(1) m} G_{m n} F_{\rho \sigma}^{(1) n}+\frac{1}{2} \operatorname{Tr}\left(\nabla_{\mu} G \nabla^{\mu} G^{-1} \nabla_{\nu} B G^{-1} \nabla^{\nu} B G^{-1}\right)\right. \\
& \quad+\operatorname{Tr}\left(\nabla_{\mu} B G^{-1} \nabla^{\mu} B G^{-1} \nabla_{\nu} B G^{-1} \nabla^{\nu} B G^{-1}\right)+\frac{1}{8} \operatorname{Tr}\left(\nabla_{\mu} G^{-1} \nabla_{\nu} G \nabla^{\mu} G^{-1} \nabla^{\nu} G\right) \\
& \quad-\frac{1}{8} \operatorname{Tr}\left(\nabla_{\mu} G^{-1} \nabla_{\nu} G\right) \operatorname{Tr}\left(\nabla^{\mu} G^{-1} \nabla^{\nu} G\right)-\frac{1}{4} \operatorname{Tr}\left(\nabla_{\mu} B G^{-1} \nabla_{\nu} B G^{-1}\right) \operatorname{Tr}\left(\nabla^{\mu} G^{-1} \nabla^{\nu} G\right)
\end{aligned}
$$




$$
\begin{aligned}
& +\frac{1}{4} H_{\mu \nu m} G^{m n} H_{\rho \sigma n} H^{\mu \nu}{ }_{p} G^{p q} H_{q}^{\rho \sigma}+\frac{1}{8} F_{\mu \nu}^{(1) m} G_{m n} F_{\rho \sigma}^{(1) n} F^{(1) \mu \rho p} G_{p q} F^{(1) \nu \sigma q} \\
& -\frac{1}{2} F_{\mu \nu}^{(1) m} G_{m n} F^{(1) \mu \rho n} F_{\rho \sigma}^{(1) p} G_{p q} F^{(1) \nu \sigma q}-H_{\mu \nu m} G^{m n} H^{\mu \rho}{ }_{n} F_{\rho \sigma}^{(1) p} G_{p q} F^{(1) \nu \sigma q} \\
& +\frac{1}{8} F_{\mu \nu}^{(1) m} H_{\rho \sigma m} F^{(1) \mu \nu n} H^{\rho \sigma}{ }_{n}+\frac{1}{4} H^{2 \mu \nu} \operatorname{Tr}\left(\nabla_{\mu} G^{-1} \nabla_{\nu} G\right)-\frac{1}{2} H_{\mu \nu}^{2} F^{(1) \mu \rho m} G_{m n} F^{(1) \nu}{ }_{\rho}{ }^{2} \\
& +\frac{1}{2} \operatorname{Tr}\left(\nabla_{\mu} B G^{-1} \nabla_{\nu} B G^{-1}\right) F^{(1) \mu \rho m} G_{m n} F^{(1) \nu}{ }_{\rho}^{n}+\frac{1}{2} \operatorname{Tr}\left(\nabla_{\mu} G^{-1} \nabla_{\nu} G\right) H^{\mu \rho}{ }_{m} G^{m n} H_{\rho n}^{\nu} \\
& +\frac{1}{2} \operatorname{Tr}\left(\nabla_{\mu} G^{-1} \nabla_{\nu} G\right) F^{(1) \mu \rho m} G_{m n} F^{(1) \nu}{ }_{\rho}{ }^{n}+\frac{1}{2} H^{\mu \nu \lambda} H^{\rho \sigma}{ }_{\lambda} H_{\mu \nu m} G^{m n} H_{\rho \sigma n} \\
& -2 H^{\mu \nu \rho} H_{\mu \nu m}\left(G^{-1} \nabla^{\sigma} B G^{-1}\right)^{m n} H_{\rho \sigma n}-\frac{1}{2} H^{\mu \nu \rho} H_{\mu \nu m}\left(G^{-1} \nabla^{\sigma} G\right)^{m}{ }_{n} F_{\rho \sigma}^{(1) n} \\
& -\frac{1}{4} F_{\mu \nu}^{(1) m}\left(\nabla_{\rho} B G^{-1} \nabla^{\rho} B\right)_{m n} F^{(1) \mu \nu n}-\frac{1}{4} H_{\mu \nu m}\left(\nabla_{\rho} G^{-1} \nabla^{\rho} G G^{-1}\right)^{m n} H^{\mu \nu}{ }_{n} \\
& -H_{\mu \nu m}\left(G^{-1} \nabla_{\rho} B G^{-1} \nabla^{\rho} B G^{-1}\right)^{m n} H^{\mu \nu}{ }_{n}-\frac{1}{2} F_{\mu \nu}^{(1) m}\left(\nabla_{\rho} G \nabla^{\nu} G^{-1} G\right)_{m n} F^{(1) \mu \rho n} \\
& \left.-2 H_{\mu \rho m}\left(G^{-1} \nabla^{\mu} B G^{-1} \nabla_{\nu} B G^{-1}\right)^{m n} H^{\nu \rho}{ }_{n}-H_{\mu \rho m}\left(G^{-1} \nabla^{\mu} B G^{-1} \nabla_{\nu} G\right)_{n}^{m} F^{(1) \nu \rho n}\right] .
\end{aligned}
$$

\section{Reduction of $\hat{\boldsymbol{R}}_{\hat{\mu} \hat{\nu} \hat{\rho} \hat{\sigma}} \hat{\boldsymbol{H}}^{\hat{\mu} \hat{\nu} \hat{\lambda}} \hat{\boldsymbol{H}}^{\hat{\rho} \hat{\sigma}_{\hat{\lambda}}}$}

Let us finally consider the reduction of the term $R H H$. The index split gives

$$
\begin{aligned}
\hat{R}_{\hat{\mu} \hat{\nu} \hat{\rho} \hat{\sigma}} \hat{H}^{\hat{\mu} \hat{\nu} \hat{\lambda}} \hat{H}^{\hat{\rho} \hat{\sigma}}{ }_{\hat{\lambda}} & =\hat{R}_{\mu \nu \rho \sigma} \hat{H}^{\mu \nu \lambda} \hat{H}^{\rho \sigma}{ }_{\lambda}+\hat{R}_{\mu \nu \rho \sigma} \hat{H}^{\mu \nu}{ }_{m} \hat{H}^{\rho \sigma m}-4 \hat{R}_{\mu \nu \rho m} \hat{H}^{\mu \nu \lambda} \hat{H}^{\rho}{ }_{\lambda}{ }^{m} \\
& -4 \hat{R}_{\mu \nu \rho m} \hat{H}^{\rho n m} \hat{H}^{\mu \nu}{ }_{n}+2 \hat{R}_{\mu \nu m n} \hat{H}^{\mu \nu \rho} \hat{H}_{\rho}{ }^{m n}+4 \hat{R}_{\mu m \nu n} \hat{H}^{\mu \rho m} \hat{H}_{\rho}{ }^{n} \\
& +4 \hat{R}_{\mu m \nu n} \hat{H}^{\mu m p} \hat{H}^{\nu n}{ }_{p}-4 \hat{R}_{\mu m n p} \hat{H}^{\mu \nu m} \hat{H}_{\nu}{ }^{n p}+\hat{R}_{m n p q} \hat{H}^{\mu m n} \hat{H}_{\mu}{ }^{p q} \\
& +2 \hat{R}_{\mu \nu m n} \hat{H}^{\mu \nu}{ }_{p} \hat{H}^{m n p}+4 \hat{R}_{\mu m n p} \hat{H}^{\mu m}{ }_{q} \hat{H}^{n p q}+\hat{R}_{m n p q} \hat{H}^{m n}{ }_{r} \hat{H}^{p q r} .
\end{aligned}
$$

Then, using Eqs. (2.5) and (4.2), the reduction of the corresponding term in the action (4.1) gives

$$
\begin{aligned}
- & \frac{1}{8} \alpha^{\prime} \int \mathrm{d}^{D+d} X \sqrt{-\hat{g}} e^{-\hat{\phi}} \hat{R}_{\hat{\mu} \hat{\nu} \hat{\rho} \hat{\sigma}} \hat{H}^{\hat{\mu} \hat{\nu} \hat{\lambda}} \hat{H}^{\hat{\rho} \hat{\sigma}} \hat{\imath} \\
\rightarrow & \frac{\alpha^{\prime}}{4} \int \mathrm{d}^{D} x \sqrt{-g} e^{-\Phi}\left[-\frac{1}{2} R_{\mu \nu \rho \sigma} H^{\mu \nu \lambda} H_{\lambda}^{\rho \sigma}-\frac{1}{2} R_{\mu \nu \rho \sigma} H^{\mu \nu}{ }_{m} G^{m n} H^{\rho \sigma}{ }_{n}\right. \\
& -\frac{1}{4} \operatorname{Tr}\left(\nabla_{\mu} G^{-1} \nabla_{\nu} B \nabla^{\mu} G^{-1} \nabla^{\nu} B\right)-\operatorname{Tr}\left(\nabla_{\mu} B \nabla^{\mu} G^{-1} G \nabla_{\nu} G^{-1} \nabla^{\nu} B G^{-1}\right) \\
& -\frac{1}{2} \operatorname{Tr}\left(\nabla_{\mu} G^{-1} G \nabla_{\nu} G^{-1} \nabla^{\mu} B G^{-1} \nabla^{\nu} B\right)+\frac{1}{4} F_{\mu \nu}^{(1) m} G_{m n} F_{\rho \sigma}^{(1) n} H^{\mu \nu}{ }_{p} G^{p q} H^{\rho \sigma}{ }_{q} \\
& +\frac{1}{4} F_{\mu \nu}^{(1) m} G_{m n} F_{\rho \sigma}^{(1) n} H^{\mu \rho}{ }_{p} G^{p q} H^{\nu \sigma}{ }_{q}-\frac{1}{2} F_{\mu \nu}^{(1) m} H_{\rho \sigma m} F^{(1) \rho \nu n} H^{\mu \sigma}{ }_{n}+\frac{1}{4} H^{\mu \nu \lambda} H^{\rho \sigma}{ }_{\lambda} F_{\mu \rho}^{(1) m} G_{m n} F_{\nu \sigma}^{(1) n} \\
& +\frac{1}{4} H^{\mu \nu \lambda} H^{\rho \sigma}{ }_{\lambda} F_{\mu \nu}^{(1) m} G_{m n} F_{\rho \sigma}^{(1) n}-H^{\mu \nu \rho} F_{\mu \nu}^{(1) m}\left(G \nabla^{\sigma} G^{-1}\right)_{m}{ }^{n} H_{\rho \sigma n}-H^{\mu \nu \rho} F_{\mu \sigma}^{(1) m}\left(G \nabla_{\nu} G^{-1}\right)_{m}{ }^{n} H_{\rho}{ }_{n} \\
& -\frac{1}{2} H^{\mu \nu \rho} F_{\mu \sigma}^{(1) m} \nabla_{\nu} B_{m n} F_{\rho}^{(1) \sigma n}-F_{\mu \nu}^{(1) m}\left(\nabla_{\rho} B \nabla^{\nu} G^{-1}\right)_{m}{ }^{n} H^{\mu \rho}{ }_{n}+\frac{1}{2} F_{\mu \nu}^{(1) m}\left(\nabla_{\rho} B G^{-1} \nabla^{\nu} B\right)_{m n} F^{(1) \mu \rho n} \\
& -F_{\mu \nu}^{(1) m}\left(G \nabla_{\rho} G^{-1} \nabla^{\rho} B G^{-1}\right)_{m}{ }^{n} H^{\mu \nu}{ }_{n}-F_{\mu \nu}^{(1) m}\left(G \nabla_{\rho} G^{-1} \nabla^{\nu} B G^{-1}\right)_{m}{ }^{n} H^{\mu \rho}{ }_{n}
\end{aligned}
$$




$$
\begin{aligned}
& -\frac{1}{2} H_{\mu \nu m}\left(\nabla_{\rho} G^{-1} \nabla^{\nu} G G^{-1}\right)^{m n} H_{n}^{\mu \rho}-H_{\mu \rho m}\left(\nabla^{\mu} G^{-1} \nabla_{\nu} G G^{-1}\right)^{m n} H_{n}^{\nu \rho} \\
& -\frac{1}{2} H^{\mu \nu \rho} \operatorname{Tr}\left(\nabla_{\mu} G^{-1} G \nabla_{\nu} G^{-1} \nabla_{\rho} B\right)+\operatorname{Tr}\left(\nabla_{\mu} \nabla_{\nu} G^{-1} \nabla^{\mu} B G^{-1} \nabla^{\nu} B\right)-H_{m}^{\mu \rho} \nabla_{\mu} \nabla_{\nu} G^{m n} H_{\rho n}^{\nu} \\
& \left.-2 \nabla_{\mu} F_{\nu \rho}^{(1) m}\left(\nabla^{\rho} B G^{-1}\right)_{m}{ }^{n} H^{\mu \nu}{ }_{n}+2 H^{\mu \nu \lambda} \nabla_{\mu} F_{\nu \rho}^{(1) m} H_{\lambda m}^{\rho}\right] .
\end{aligned}
$$

Apart from the Riemann tensor, the four last terms contain second-order derivatives. Just as for the Riemann squared term (4.8), upon partial integration, one can transform these terms such that all second-order derivatives appear as the leading two-derivative contribution from the field Eqs. (2.17). Details are given in Appendix B. Specifically, the remaining secondorder derivative terms combine into

$$
\begin{aligned}
\int & \mathrm{d}^{D} x \sqrt{-g} e^{-\Phi} \frac{\alpha^{\prime}}{4}\left[\frac{1}{2} \operatorname{Tr}\left(\square G^{-1} \nabla_{\nu} B G^{-1} \nabla^{\nu} B\right)-\operatorname{Tr}\left(\square B G^{-1} \nabla_{\nu} B \nabla^{\nu} G^{-1}\right)\right. \\
& -\frac{1}{2} F_{\mu \nu}^{(1) m}\left(\square B G^{-1}\right)_{m}{ }^{n} H^{\mu \nu}{ }_{n}-\frac{1}{4} H^{\mu \nu}{ }_{m} \square G^{m n} H_{\mu \nu n}-\nabla_{\mu} H^{\mu \nu \rho} F_{\nu \sigma}^{(1) m} H^{\sigma}{ }_{\rho m} \\
& -\frac{1}{2} \nabla_{\mu} H^{\mu \nu}{ }_{m}\left(2 \nabla^{\rho} G^{m n} H_{\nu \rho n}-H_{\nu \rho \sigma} F^{(1) \rho \sigma m}+2\left(G^{-1} \nabla^{\rho} B\right)^{m}{ }_{n} F_{\nu \rho}^{(1) n}\right) \\
& \left.-\frac{1}{2} \nabla_{\mu} F^{(1) \mu \nu m}\left(2\left(\nabla^{\rho} B G^{-1}\right)_{m}{ }^{n} H_{\nu \rho n}-H_{\nu \rho \sigma} H_{m}^{\rho \sigma}\right)\right],
\end{aligned}
$$

and can be eliminated by field redefinitions according to the rules defined in Table I. The explicit induced field redefinitions are collected in Eq. (B9). The final result of the reduction (4.12) then takes the form

$$
\begin{aligned}
& -\frac{1}{8} \alpha^{\prime} \int \mathrm{d}^{D+d} X \sqrt{-\hat{g}} e^{-\hat{\phi}} \hat{R}_{\hat{\mu} \hat{\nu} \hat{\rho} \hat{\sigma}} \hat{H}^{\hat{\mu} \hat{\nu} \hat{\lambda}} \hat{H}^{\hat{\rho} \hat{\sigma}} \hat{\lambda} \\
& \rightarrow \frac{\alpha^{\prime}}{4} \int \mathrm{d}^{D} x \sqrt{-g} e^{-\Phi}\left[-\frac{1}{2} R_{\mu \nu \rho \sigma} H^{\mu \nu \lambda} H^{\rho \sigma}{ }_{\lambda}-\frac{1}{2} R_{\mu \nu \rho \sigma} H^{\mu \nu}{ }_{m} G^{m n} H^{\rho \sigma}{ }_{n}+\frac{1}{4} \operatorname{Tr}\left(\nabla_{\mu} G^{-1} \nabla_{\nu} B \nabla^{\mu} G^{-1} \nabla^{\nu} B\right)\right. \\
& -\frac{1}{2} \operatorname{Tr}\left(\nabla_{\mu} G \nabla^{\mu} G^{-1} \nabla_{\nu} B G^{-1} \nabla^{\nu} B G^{-1}\right)-\frac{1}{2} \operatorname{Tr}\left(\nabla_{\mu} B G^{-1} \nabla^{\mu} B G^{-1} \nabla_{\nu} B G^{-1} \nabla^{\nu} B G^{-1}\right) \\
& -\frac{1}{2} \operatorname{Tr}\left(\nabla_{\mu} G^{-1} G \nabla_{\nu} G^{-1} \nabla^{\mu} B G^{-1} \nabla^{\nu} B\right)-\frac{1}{8} H_{\mu \nu m} G^{m n} H_{\rho \sigma n} H_{p}^{\mu \nu} G^{p q} H_{q}^{\rho \sigma}+\frac{1}{4} F_{\mu \nu}^{(1) m} G_{m n} F_{\rho \sigma}^{(1) n} H_{p}^{\mu \rho} G^{p q} H^{\nu \sigma}{ }_{q} \\
& +\frac{1}{2} F_{\mu \nu}^{(1) m} H_{\rho \sigma m} F^{(1) \rho \nu n} H_{n}^{\mu \sigma}-\frac{1}{8} F_{\mu \nu}^{(1) m} H_{\rho \sigma m} F^{(1) \mu \nu n} H_{n}^{\rho \sigma}+\frac{1}{4} H^{\mu \nu \lambda} H_{\lambda}^{\rho \sigma} F_{\mu \rho}^{(1) m} G_{m n} F_{\nu \sigma}^{(1) n}-\frac{1}{4} H^{\mu \nu \lambda} H^{\rho \sigma} H_{\mu \nu m} G^{m n} H_{\rho \sigma n} \\
& +H^{\mu \nu \rho} H_{\mu \nu m}\left(G^{-1} \nabla^{\sigma} B G^{-1}\right)^{m n} H_{\rho \sigma n}+\frac{1}{2} H^{\mu \nu \rho} H_{\mu \nu m}\left(G^{-1} \nabla^{\sigma} G\right)^{m}{ }_{n} F_{\rho \sigma}^{(1) m} \\
& -H^{\mu \nu \rho} F_{\mu \sigma}^{(1) m}\left(G \nabla_{\nu} G^{-1}\right)_{m}{ }^{n} H_{\rho}{ }{ }_{n}+\frac{1}{2} H^{\mu \nu \rho} F_{\mu \sigma}^{(1) m} \nabla_{\nu} B_{m n} F_{\rho}^{(1) \sigma n} \\
& +\frac{1}{4} F_{\mu \nu}^{(1) m}\left(\nabla_{\rho} B G^{-1} \nabla^{\rho} B\right)_{m n} F^{(1) \mu \nu n}+\frac{1}{2} H_{\mu \nu m}\left(G^{-1} \nabla_{\rho} B G^{-1} \nabla^{\rho} B G^{-1}\right)^{m n} H^{\mu \nu}{ }_{n} \\
& +\frac{1}{4} H_{\mu \nu m}\left(G^{-1} \nabla_{\rho} G \nabla^{\rho} G^{-1}\right)^{m n} H^{\mu \nu}{ }_{n}-F_{\mu \nu}^{(1) m}\left(G \nabla_{\rho} G^{-1} \nabla^{\nu} B G^{-1}\right)_{m}{ }^{n} H^{\mu \rho}{ }_{n} \\
& +F_{\mu \nu}^{(1) m}\left(\nabla_{\rho} B \nabla^{\nu} G^{-1}\right)_{m}{ }^{n} H^{\mu \rho}{ }_{n}-\frac{1}{2} F_{\mu \nu}^{(1) m}\left(\nabla_{\rho} B G^{-1} \nabla^{\nu} B\right)_{m n} F^{(1) \mu \rho n} \\
& -\frac{1}{2} H_{\mu \nu m}\left(\nabla_{\rho} G^{-1} \nabla^{\nu} G G^{-1}\right)^{m n} H_{n}^{\mu \rho}+H_{\mu \rho m}\left(G^{-1} \nabla^{\mu} B G^{-1} \nabla_{\nu} B G^{-1}\right)^{m n} H_{n}^{\nu \rho} \\
& \left.-F_{\mu \rho}^{(1) m}\left(\nabla^{\mu} G G^{-1} \nabla_{\nu} B G^{-1}\right)_{m}{ }^{n} H^{\nu \rho}{ }_{n}-\frac{1}{2} H^{\mu \nu \rho} \operatorname{Tr}\left(\nabla_{\mu} G^{-1} G \nabla_{\nu} G^{-1} \nabla_{\rho} B\right)\right] \text {. }
\end{aligned}
$$

In the next section, we will match the result of the explicit reduction against the basis (3.26) in order to establish the $\mathrm{O}(d, d, \mathbb{R})$ invariance of the reduced action. 


\section{B. Field redefinitions}

By partial integration and suitable field redefinitions, we have thus cast the reduced action at order $\alpha^{\prime}$ into a form which is polynomial in first-order derivatives and the Riemann tensor. As an illustration and for potential applications requiring the dictionary between the lower-dimensional fields and the fields featuring in the original action (2.1), let us list the full set of induced field redefinitions, put together from Eqs. (B4) and (B9):

$$
\begin{aligned}
\delta \Phi= & \frac{1}{4}\left[-F_{\mu \nu}^{(1) m} G_{m n} F^{(1) \mu \nu n}+\frac{1}{2} \operatorname{Tr}\left(\nabla_{\mu} G^{-1} \nabla^{\mu} G\right)\right], \\
\delta g_{\mu \nu}= & \frac{1}{4}\left[2 F_{\mu \rho}^{(1) m} G_{m n} F_{\nu}^{(1) \rho n}-\operatorname{Tr}\left(\nabla_{(\mu} G^{-1} \nabla_{\nu)} G\right)\right], \\
\delta B_{\mu \nu}= & \frac{1}{8}\left[\left(-2 \nabla^{\rho} F_{\rho \mu}^{(1) m}+2 \nabla^{\rho} \Phi F_{\rho \mu}^{(1) m}+\frac{1}{2} H_{\mu \rho \sigma} H^{\rho \sigma}{ }_{p} G^{p m}\right.\right. \\
& \left.+F_{\mu \rho}^{(1) p}\left(\nabla^{\rho} G G^{-1}\right)_{p}{ }^{m}+H_{\mu \rho p}\left(G^{-1} \nabla^{\rho} B G^{-1}\right)^{p m}\right)\left(A_{\nu m}^{(2)}-B_{m n} A_{\nu}^{(1) n}\right) \\
& -A_{\mu}^{(1) m}\left(G \nabla_{\rho} G^{-1}\right)_{m}{ }^{n} H_{\nu \rho n}-A_{\mu}^{(1) m} \nabla^{\rho} B_{m n} F_{\nu \rho}^{(1) n}+2 F_{\mu \rho}^{(1) m} H^{\rho}{ }_{\nu m} \\
& \left.+\frac{1}{2} A_{\mu}^{(1) m} H_{\nu \rho \sigma} G_{m n} F^{(1) \rho \sigma n}\right]-(\mu \leftrightarrow \nu), \\
\delta G^{m n}= & \frac{1}{4}\left[-2 \square G^{m n}+2 \nabla_{\mu} \Phi \nabla^{\mu} G^{m n}-\frac{1}{2} G^{m p} H_{\mu \nu p} G^{n q} H^{\mu \nu}{ }_{q}-\frac{3}{2} F_{\mu \nu}^{(1) m} F^{(1) \mu \nu n}\right. \\
& \left.-\left(G^{-1} \nabla_{\mu} G \nabla^{\mu} G^{-1}\right)^{m n}+\left(G^{-1} \nabla_{\mu} B G^{-1} \nabla^{\mu} B G^{-1}\right)^{m n}\right], \\
\delta B_{m n}= & \frac{1}{4}\left[\left(\nabla_{\mu} B \nabla^{\mu} G^{-1} G\right)_{m n}+\left(G \nabla_{\mu} G^{-1} \nabla^{\mu} B\right)_{m n}-\frac{1}{2} H_{\mu \nu m} F^{(1) \mu \nu p} G_{p n}+\frac{1}{2} G_{m p} F^{(1) \mu \nu p} H_{\mu \nu n}\right], \\
\delta A_{\mu}^{(1) m}= & \frac{1}{4}\left[-2 \nabla^{\nu} F_{\nu \mu}^{(1) m}+2 \nabla^{\nu} \Phi F_{\nu \mu}^{(1) m}+\frac{1}{2} H_{\mu \nu \rho} H^{\nu \rho}{ }_{n} G^{n m}+F_{\mu \nu}^{(1) n}\left(\nabla^{\nu} G G^{-1}\right)_{n}{ }^{m}+H_{\mu \nu n}\left(G^{-1} \nabla^{\nu} B G^{-1}\right)^{n m}\right], \\
\delta A_{\mu m}^{(2)}= & \frac{1}{4}\left[2 \nabla^{\nu} F_{\nu \mu}^{(1) n} B_{n m}-2 \nabla^{\nu} \Phi F_{\nu \mu}^{(1) n} B_{n m}-\frac{1}{2} H_{\mu \nu \rho} H^{\nu \rho}{ }_{n}\left(G^{-1} B\right)^{n}{ }_{m}-F_{\mu \nu}^{(1) n}\left(\nabla^{\nu} G G^{-1} B\right)_{n m}\right. \\
& \left.-H_{\mu \nu n}\left(G^{-1} \nabla^{\nu} B G^{-1} B\right)^{n}{ }_{m}+H_{\mu \nu n}\left(\nabla^{\nu} G^{-1} G\right)^{n}{ }_{m}-F_{\mu \nu}^{(1) n} \nabla^{\nu} B_{n m}-\frac{1}{2} H_{\mu \nu \rho} F^{(1) \nu \rho n} G_{n m}\right],
\end{aligned}
$$

where we used the convention of Eq. (2.15).

\section{O $(d, d, \mathbb{R})$ INVARIANCE AND A GREEN-SCHWARZ TYPE MECHANISM}

We have now set up all the elements allowing one to systematically exhibit the $\mathrm{O}(d, d, \mathbb{R})$ invariance of the dimensionally reduced theory at order $\alpha^{\prime}$. Having brought the reduced action into a form that is polynomial in first derivatives (and the Riemann tensor), we have fully fixed the ambiguities due to field redefinitions and partial integration. We can then compare the result to the distinguished manifestly $\mathrm{O}(d, d, \mathbb{R})$ invariant basis constructed in Sec. III D, after breaking up the latter under $\mathrm{GL}(d) .{ }^{5}$ Different terms of the $\mathrm{O}(d, d, \mathbb{R})$ basis (3.26) do

\footnotetext{
${ }^{5}$ See Appendix $\mathrm{C}$ for the $\mathrm{GL}(d)$ expressions of the relevant $\mathrm{O}(d, d, \mathbb{R})$ terms.
}

not share common terms in the decomposition under $\mathrm{GL}(d)$; i.e., every $\mathrm{GL}(d)$ invariant term we have obtained in the reduction in the previous section has a unique ancestor within the $\mathrm{O}(d, d, \mathbb{R})$ basis (3.26). It becomes thus a straightforward-albeit lengthy - task to recombine (if possible) any collection of $\mathrm{GL}(d)$ terms into $\mathrm{O}(d, d, \mathbb{R})$ invariant expressions.

The dimensionally reduced action is given by the sum of Eqs. (4.4), (4.6), (4.10), and (4.14). Upon combining these terms into the $\mathrm{O}(d, d, \mathbb{R})$ invariant expressions of the basis (3.26), we can bring it into the form

$$
I_{1}=\underline{I}_{1}+O_{1},
$$

where $\underline{I}_{1}$ is the part of $I_{1}$ that can be organized into a linear combination of manifestly $\mathrm{O}(d, d, \mathbb{R})$ invariant basis elements as 


$$
\begin{aligned}
I_{1}= & \frac{1}{4} \alpha^{\prime} \int \mathrm{d}^{D} x \sqrt{-g} e^{-\Phi}\left[R_{\mu \nu \rho \sigma} R^{\mu \nu \rho \sigma}-\frac{1}{2} R_{\mu \nu \rho \sigma} H^{\mu \nu \lambda} H^{\rho \sigma}{ }_{\lambda}-\frac{1}{8} H_{\mu \nu}^{2} H^{2 \mu \nu}+\frac{1}{24} H_{\mu \nu \rho} H^{\mu}{ }_{\sigma}{ }^{\lambda} H^{\nu}{ }_{\lambda}{ }^{\tau} H^{\rho}{ }_{\tau}{ }^{\sigma}\right. \\
& -\frac{1}{2} R_{\mu \nu \rho \sigma} \mathcal{F}^{\mu \nu M} \mathcal{S}_{M}{ }^{N} \mathcal{F}^{\rho \sigma}{ }_{N}+\frac{1}{16} \operatorname{Tr}\left(\nabla_{\mu} \mathcal{S} \nabla_{\nu} \mathcal{S} \nabla^{\mu} \mathcal{S} \nabla^{\nu} \mathcal{S}\right)-\frac{1}{32} \operatorname{Tr}\left(\nabla_{\mu} \mathcal{S} \nabla_{\nu} \mathcal{S}\right) \operatorname{Tr}\left(\nabla^{\mu} \mathcal{S} \nabla^{\nu} \mathcal{S}\right) \\
& +\frac{1}{8} \mathcal{F}_{\mu \nu}{ }^{M} \mathcal{F}_{\rho \sigma} \mathcal{F}^{\mu \rho N} \mathcal{F}^{\nu \sigma}{ }_{N}-\frac{1}{2} \mathcal{F}_{\mu \nu}{ }^{M} \mathcal{S}_{M}{ }^{N} \mathcal{F}^{\mu \rho}{ }_{N} \mathcal{F}^{\nu \sigma P} \mathcal{S}_{P} Q \mathcal{F}_{\rho \sigma Q} Q \\
& +\frac{1}{8} \mathcal{F}_{\mu \nu}{ }^{M} \mathcal{S}_{M}{ }^{N} \mathcal{F}_{\rho \sigma N} \mathcal{F}^{\mu \rho P} \mathcal{S}_{P}{ }^{Q} \mathcal{F}^{\nu \sigma}{ }_{Q}+\frac{1}{8} H_{\mu \nu}^{2} \operatorname{Tr}\left(\nabla^{\mu} \mathcal{S} \nabla^{\nu} \mathcal{S}\right)-\frac{1}{2} H_{\mu \nu}^{2} \mathcal{F}^{\mu}{ }_{\rho}{ }^{M} \mathcal{S}_{M}{ }^{N} \mathcal{F}^{\nu \rho}{ }_{N} \\
& +\frac{1}{4} \mathcal{F}^{\mu \rho M} \mathcal{S}_{M}{ }^{N} \mathcal{F}^{\nu}{ }_{\rho N} \operatorname{Tr}\left(\nabla_{\mu} \mathcal{S} \nabla_{\nu} \mathcal{S}\right)+\frac{1}{4} H^{\mu \nu \lambda} H^{\rho \sigma}{ }_{\lambda} \mathcal{F}_{\mu \rho}{ }^{M} \mathcal{S}_{M}{ }^{N} \mathcal{F}_{\nu \sigma N} \\
& \left.-\frac{1}{2} H^{\mu \nu \rho} \mathcal{F}_{\mu \sigma}{ }^{M}\left(\mathcal{S} \nabla_{\nu} \mathcal{S}\right)_{M}{ }^{N} \mathcal{F}_{\rho}{ }^{\sigma}{ }_{N}-\frac{1}{2} \mathcal{F}_{\mu \nu}{ }^{M}\left(\mathcal{S} \nabla_{\rho} \mathcal{S} \nabla^{\nu} \mathcal{S}\right)_{M}{ }^{N} \mathcal{F}^{\mu \rho}{ }_{N}\right]
\end{aligned}
$$

whereas the remaining part of the action $O_{1}$ is not manifestly $\mathrm{O}(d, d, \mathbb{R})$ invariant, but given by

$$
O_{1}=-\frac{1}{8} \alpha^{\prime} \int \mathrm{d}^{D} x \sqrt{-g} e^{-\Phi} H^{\mu \nu \rho} \operatorname{Tr}\left[\nabla_{\mu} G^{-1} G \nabla_{\nu} G^{-1} \nabla_{\rho} B-\frac{1}{3} \nabla_{\mu} B G^{-1} \nabla_{\nu} B G^{-1} \nabla_{\rho} B G^{-1}\right] .
$$

This suggests the definition

$$
\Omega_{\mu \nu \rho}=-\frac{3}{4} \operatorname{Tr}\left(\partial_{[\mu} G^{-1} G \partial_{\nu} G^{-1} \partial_{\rho]} B\right)+\frac{1}{4} \operatorname{Tr}\left(\partial_{[\mu} B G^{-1} \partial_{\nu} B G^{-1} \partial_{\rho]} B G^{-1}\right),
$$

such that $O_{1}$ takes the form

$$
O_{1}=\frac{1}{6} \alpha^{\prime} \int \mathrm{d}^{D} x \sqrt{-g} e^{-\Phi} H_{\mu \nu \rho} \Omega^{\mu \nu \rho} .
$$

The 3-form (5.4) descends from the nonvanishing cohomology $H^{4}$ of $\mathrm{O}(d, d, \mathbb{R}) /(\mathrm{O}(d) \times \mathrm{O}(d))[32,33]$; although it is not $\mathrm{O}(d, d, \mathbb{R})$ invariant, its exterior derivative is ${ }^{6}$

$$
4 \partial_{[\mu} \Omega_{\nu \rho \sigma]}=\frac{3}{8} \operatorname{Tr}\left(\mathcal{S} \partial_{[\mu} \mathcal{S} \partial_{\nu} \mathcal{S} \partial_{\rho} \mathcal{S} \partial_{\sigma]} \mathcal{S}\right) .
$$

For $\Gamma \in \mathfrak{v}(d, d, \mathbb{R})$ this implies that $\mathrm{d} \delta_{\Gamma} \Omega=\delta_{\Gamma} \mathrm{d} \Omega=0$; i.e., the $\mathrm{O}(d, d, \mathbb{R})$ variation of $\Omega_{\mu \nu \rho}$ is closed and can locally be integrated to a 2-form $X_{\mu \nu}$ such that

$$
\delta_{\Gamma} \Omega_{\mu \nu \rho}=3 \partial_{[\mu} X_{\nu \rho]}
$$

This observation together with the particular form of (5.5) suggests a Green-Schwarz type mechanism in order to restore $\mathrm{O}(d, d, \mathbb{R})$ invariance of the $D$-dimensional action. Specifically, the term (5.5) can be absorbed into a deformation of the two-derivative action (2.11) upon redefining

$$
\tilde{H}_{\mu \nu \rho} \equiv H_{\mu \nu \rho}-\alpha^{\prime} \Omega_{\mu \nu \rho},
$$

such that the kinetic term now produces

\footnotetext{
${ }^{6}$ See Appendix C for the $\mathrm{GL}(d)$ expression.
}

$$
-\frac{1}{12} \tilde{H}^{\mu \nu \rho} \tilde{H}_{\mu \nu \rho}=-\frac{1}{12} H^{\mu \nu \rho} H_{\mu \nu \rho}+\frac{\alpha^{\prime}}{6} H^{\mu \nu \rho} \Omega_{\mu \nu \rho}+\mathcal{O}\left(\alpha^{\prime 2}\right) .
$$

In view of Eq. (5.7), the deformed field strength (5.8) remains $\mathrm{O}(d, d, \mathbb{R})$ invariant, if we impose on $B_{\mu \nu}$ a nontrivial $\mathrm{O}(d, d, \mathbb{R})$ transformation for $\Gamma \in \mathfrak{v}(d, d, \mathbb{R})$ as

$$
\delta_{\Gamma} B_{\mu \nu}=\alpha^{\prime} X_{\mu \nu} \Rightarrow \delta_{\Gamma} \tilde{H}_{\mu \nu \rho}=0
$$

The resulting theory is then fully $\mathrm{O}(d, d, \mathbb{R})$ invariant to first order in $\alpha^{\prime}$. In order to compute an explicit expression for $X_{\mu \nu}$, we start from a general $\mathfrak{o}(d, d, \mathbb{R})$ matrix parametrized as

$$
\Gamma_{M}^{N}=\left(\begin{array}{cc}
\mathfrak{a}_{m}{ }^{n} & \mathfrak{b}_{m n} \\
\mathfrak{c}^{m n} & -\mathfrak{a}_{n}{ }^{m}
\end{array}\right),
$$

with $\mathfrak{c}^{m n}$ and $\mathfrak{b}_{m n}$ antisymmetric. Further defining the $\mathfrak{v}(d, d, \mathbb{R})$ matrices

$$
\begin{aligned}
\mathfrak{A}(\mathfrak{a})_{M}{ }^{N} & =\left(\begin{array}{cc}
\mathfrak{a}_{m}{ }^{n} & 0 \\
0 & -\mathfrak{a}_{n}{ }^{m}
\end{array}\right), \\
\mathfrak{S}(\mathfrak{c})_{M}{ }^{N} & =\left(\begin{array}{cc}
0 & 0 \\
\mathfrak{c}^{m n} & 0
\end{array}\right),
\end{aligned}
$$

the $\mathfrak{o}(d, d, \mathbb{R})$ algebra takes the form 


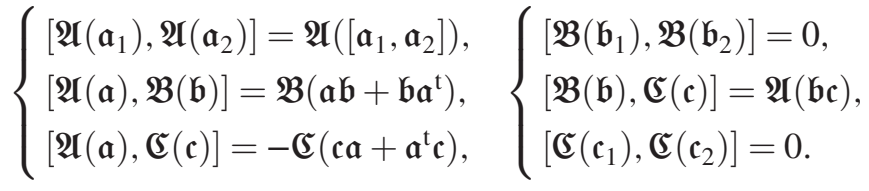

The action of these generators on $G_{m n}$ and $B_{m n}$ is obtained from Eq. (2.9) as

$$
\left\{\begin{array}{l}
\delta_{\Gamma} G=\mathfrak{a} G+G \mathfrak{a}^{\mathrm{t}}-G \mathfrak{c} B-B \mathfrak{c} G, \\
\delta_{\Gamma} B=\mathfrak{a} B+B \mathfrak{a}^{\mathrm{t}}-B \mathfrak{c} B-G \mathfrak{c} G+\mathfrak{b},
\end{array}\right.
$$

which, together with Eq. (5.4), yields the general $\mathrm{O}(d, d, \mathbb{R})$ variation of $\Omega_{\mu \nu \rho}$,

$$
\begin{aligned}
\delta_{\Gamma} \Omega_{\mu \nu \rho}= & -\frac{3}{2}\left[\operatorname{Tr}\left(\mathfrak{c} \partial_{[\mu} G \partial_{\nu} G^{-1} \partial_{\rho]} G\right)\right. \\
& \left.+\operatorname{Tr}\left(\mathfrak{c} \partial_{[\mu} B \partial_{\nu} G^{-1} \partial_{\rho]} B\right)\right] .
\end{aligned}
$$

Pulling out one derivative, we extract the explicit form of $X_{\mu \nu}$ from Eq. (5.7):

$$
X_{\mu \nu}=\frac{1}{2} \operatorname{Tr}\left(\mathfrak{c} \partial_{[\mu}(G+B) G^{-1} \partial_{\nu]}(G+B)\right) .
$$

According to Eq. (5.10), the 2-form thus acquires new transformations only along the nilpotent $\mathfrak{o}(d, d, \mathbb{R})$ generators $\mathfrak{c}^{m n}$. This is consistent with the fact that all the other $\mathfrak{o}(d, d, \mathbb{R})$ generators have a geometric origin and by construction represent manifest symmetries of the dimensionally reduced action. Moreover, with the expression (5.16), one can verify that the algebra of $\mathfrak{o}(d, d, \mathbb{R})$ transformations (5.13) closes on $B_{\mu \nu}$. Crucially, the deformed $\mathfrak{v}(d, d, \mathbb{R})$ action (5.10) cannot be absorbed into a redefinition of the fields but represents a genuine deformation of the $\mathrm{O}(d, d, \mathbb{R})$ transformation rules.

We may also consider the behavior of Eq. (5.5) under the $\mathbb{Z}_{2}$ invariance of bosonic string theory that sends $\hat{B} \rightarrow-\hat{B}$. On the $\mathrm{O}(d, d, \mathbb{R})$ matrix (2.9) this symmetry acts as [15]

$$
\mathcal{H} \rightarrow Z^{T} \mathcal{H} Z, \quad Z \equiv\left(\begin{array}{cc}
1 & 0 \\
0 & -1
\end{array}\right) .
$$

The matrix $Z$ is not $\mathrm{O}(d, d, \mathbb{R})$ valued since the metric (2.10) transforms as

$$
\eta \rightarrow Z \eta Z^{T}=-\eta \quad \Rightarrow \quad \mathcal{S} \rightarrow-Z \mathcal{S} Z .
$$

Thus, the $\mathrm{O}(d, d, \mathbb{R})$ invariant defined by the right-hand side of Eq. (5.6) is $\mathbb{Z}_{2}$ odd. This ensures $\mathbb{Z}_{2}$ invariance of the action (5.5) since $B_{\mu \nu}$ and its field strength $H_{\mu \nu \rho}$ are also $\mathbb{Z}_{2}$ odd.

Let us summarize the previous discussion. The bosonic string effective action, including its first-order $\alpha^{\prime}$-corrections, upon compactification on a $d$-torus exhibits a global $\mathrm{O}(d, d, \mathbb{R})$ symmetry, provided the $\mathrm{O}(d, d, \mathbb{R})$ transformations of the two-derivative action acquire $\alpha^{\prime}$ corrections according to Eq. (5.10). The full $\alpha^{\prime}$-corrected transformations are given by

$$
\begin{aligned}
& \left\{\begin{array}{l}
\delta_{\Gamma} g_{\mu \nu}=0, \\
\delta_{\Gamma} B_{\mu \nu}=\frac{\alpha^{\prime}}{2} \operatorname{Tr}\left(\mathfrak{c} \partial_{[\mu}(G+B) G^{-1} \partial_{\nu]}(G+B)\right),
\end{array}\right. \\
& \left\{\begin{array}{l}
\delta_{\Gamma} \mathcal{H}_{M N}=\Gamma_{M}{ }^{P} \mathcal{H}_{P N}+\Gamma_{N}{ }^{P} \mathcal{H}_{M P}, \\
\delta_{\Gamma} \mathcal{F}_{\mu \nu}{ }^{M}=-\mathcal{F}_{\mu \nu}{ }^{N} \Gamma_{N}{ }^{M},
\end{array}\right.
\end{aligned}
$$

for $\Gamma_{M}{ }^{N} \in \mathfrak{v}(d, d, \mathbb{R})$ parametrized as Eq. (5.11). To order $\alpha^{\prime}$, the $\mathrm{O}(d, d, \mathbb{R})$ invariant action is given by

$$
\begin{aligned}
I= & \int \mathrm{d}^{D} x \sqrt{-g} e^{-\Phi}\left[R+\partial_{\mu} \Phi \partial^{\mu} \Phi-\frac{1}{12} \tilde{H}_{\mu \nu \rho} \tilde{H}^{\mu \nu \rho}+\frac{1}{8} \operatorname{Tr}\left(\partial_{\mu} \mathcal{S} \partial^{\mu} \mathcal{S}\right)-\frac{1}{4} \mathcal{F}_{\mu \nu}^{M} \mathcal{S}_{M}{ }^{N} \mathcal{F}_{N}^{\mu \nu}\right. \\
& +\frac{1}{4} \alpha^{\prime}\left(R_{\mu \nu \rho \sigma} R^{\mu \nu \rho \sigma}-\frac{1}{2} R_{\mu \nu \rho \sigma} H^{\mu \nu \lambda} H^{\rho \sigma}{ }_{\lambda}+\frac{1}{24} H_{\mu \nu \rho} H^{\mu}{ }_{\sigma}{ }^{\lambda} H^{\nu}{ }_{\lambda}{ }^{\tau} H^{\rho}{ }_{\tau}{ }^{\sigma}-\frac{1}{8} H_{\mu \nu}^{2} H^{2 \mu \nu}+\frac{1}{16} \operatorname{Tr}\left(\nabla_{\mu} \mathcal{S} \nabla_{\nu} \mathcal{S} \nabla^{\mu} \mathcal{S} \nabla^{\nu} \mathcal{S}\right)\right. \\
& -\frac{1}{32} \operatorname{Tr}\left(\nabla_{\mu} \mathcal{S} \nabla_{\nu} \mathcal{S}\right) \operatorname{Tr}\left(\nabla^{\mu} \mathcal{S} \nabla^{\nu} \mathcal{S}\right)+\frac{1}{8} \mathcal{F}_{\mu \nu}{ }^{M} \mathcal{S}_{M}{ }^{N} \mathcal{F}_{\rho \sigma N} \mathcal{F}^{\mu \rho P} \mathcal{S}_{P}{ }^{Q} \mathcal{F}^{\nu \sigma}{ }_{Q}-\frac{1}{2} \mathcal{F}_{\mu \nu}{ }^{M} \mathcal{S}_{M}{ }^{N} \mathcal{F}^{\mu \rho}{ }_{N} \mathcal{F}^{\nu \sigma P} \mathcal{S}_{P}{ } \mathcal{F}_{\rho \sigma Q} \\
& +\frac{1}{8} \mathcal{F}_{\mu \nu}{ }^{M} \mathcal{F}_{\rho \sigma}{ }_{\rho} \mathcal{F}^{\mu \rho N} \mathcal{F}^{\nu \sigma}{ }_{N}-\frac{1}{2} R_{\mu \nu \rho \sigma} \mathcal{F}^{\mu \nu M} \mathcal{S}_{M}{ }^{N} \mathcal{F}^{\rho \sigma}{ }_{N}+\frac{1}{8} H_{\mu \nu}^{2} \operatorname{Tr}\left(\nabla^{\mu} \mathcal{S} \nabla^{\nu} \mathcal{S}\right)-\frac{1}{2} H_{\mu \nu}^{2} \mathcal{F}^{\mu}{ }_{\rho}{ }^{M} \mathcal{S}_{M}{ }^{N} \mathcal{F}^{\nu \rho}{ }_{N} \\
& +\frac{1}{4} H^{\mu \nu \lambda} H^{\rho \sigma}{ }_{\lambda} \mathcal{F}_{\mu \rho}{ }^{M} \mathcal{S}_{M}{ }^{N} \mathcal{F}_{\nu \sigma N}-\frac{1}{2} \mathcal{F}_{\mu \nu}{ }^{M}\left(\mathcal{S} \nabla_{\rho} \mathcal{S} \nabla^{\nu} \mathcal{S}\right)_{M}{ }^{N} \mathcal{F}^{\mu \rho}{ }_{N}+\frac{1}{4} \mathcal{F}^{\mu \rho M} \mathcal{S}_{M}{ }^{N} \mathcal{F}^{\nu}{ }_{\rho N} \operatorname{Tr}\left(\nabla_{\mu} \mathcal{S} \nabla_{\nu} \mathcal{S}\right) \\
& \left.\left.-\frac{1}{2} H^{\mu \nu \rho} \mathcal{F}_{\mu \sigma}{ }^{M}\left(\mathcal{S} \nabla_{\nu} \mathcal{S}\right)_{M}{ }^{N} \mathcal{F}_{\rho}{ }^{\sigma}{ }_{N}\right)\right]+\mathcal{O}\left(\alpha^{2}\right),
\end{aligned}
$$

with the deformed field-strength $\tilde{H}_{\mu \nu \rho}$ defined in Eq. (5.8). This constitutes the main result of this paper.

Let us comment on the relation to Ref. [8], where a similar analysis of the first-order $\alpha^{\prime}$-corrections is performed, however, restricted to the scalar sector, i.e., setting $A_{\mu}^{(1) m}=A_{\mu m}^{(2)}=B_{\mu \nu}=0, g_{\mu \nu}=\eta_{\mu \nu}$. Their result is given in their Eq. (74): 


$$
\begin{aligned}
I_{1}= & \frac{1}{8} \alpha^{\prime} \int \mathrm{d}^{D} x e^{-\Phi}\left[-\operatorname{Tr}\left(\nabla_{\mu} \nabla_{\nu} \mathcal{S} \nabla^{\mu} \nabla^{\nu} \mathcal{S}\right)+\frac{1}{16} \operatorname{Tr}\left(\nabla_{\mu} \mathcal{S} \nabla_{\nu} \mathcal{S}\right) \operatorname{Tr}\left(\nabla^{\mu} \mathcal{S} \nabla^{\nu} \mathcal{S}\right)\right. \\
& \left.+\operatorname{Tr}\left(\nabla_{\mu} \mathcal{S} \nabla^{\mu} \mathcal{S} \nabla_{\nu} \mathcal{S} \nabla^{\nu} \mathcal{S}\right)+\frac{1}{8} \operatorname{Tr}\left(\nabla_{\mu} \mathcal{S} \nabla_{\nu} \mathcal{S} \nabla^{\mu} \mathcal{S} \nabla^{\nu} \mathcal{S}\right)\right]
\end{aligned}
$$

Upon partial integration, this can be rewritten as

$$
\begin{aligned}
I_{1}= & \frac{1}{8} \alpha^{\prime} \int \mathrm{d}^{D} x \sqrt{-g} e^{-\Phi}\left[-\operatorname{Tr}\left(\left(\square \mathcal{S}-\nabla_{\mu} \Phi \nabla^{\mu} \mathcal{S}\right)\left(\square \mathcal{S}-\nabla_{\nu} \Phi \nabla^{\nu} \mathcal{S}\right)\right)+\left(R_{\mu \nu}+\nabla_{\mu} \nabla_{\nu} \Phi\right) \operatorname{Tr}\left(\nabla^{\mu} \mathcal{S} \nabla^{\nu} \mathcal{S}\right)\right. \\
& \left.+\frac{1}{16} \operatorname{Tr}\left(\nabla_{\mu} \mathcal{S} \nabla_{\nu} \mathcal{S}\right) \operatorname{Tr}\left(\nabla^{\mu} \mathcal{S} \nabla^{\nu} \mathcal{S}\right)+\operatorname{Tr}\left(\nabla_{\mu} \mathcal{S} \nabla^{\mu} \mathcal{S} \nabla_{\nu} \mathcal{S} \nabla^{\nu} \mathcal{S}\right)+\frac{1}{8} \operatorname{Tr}\left(\nabla_{\mu} \mathcal{S} \nabla_{\nu} \mathcal{S} \nabla^{\mu} \mathcal{S} \nabla^{\nu} \mathcal{S}\right)\right] .
\end{aligned}
$$

As discussed in Sec. II B, we can then remove the second-order derivative terms by performing the $[\mathrm{O}(d, d, \mathbb{R})$ covariant $]$ field redefinitions

$$
\left\{\begin{array}{l}
\delta \Phi=\frac{1}{16} \operatorname{Tr}\left(\nabla_{\mu} \mathcal{S} \nabla^{\mu} \mathcal{S}\right) \\
\delta g_{\mu \nu}=-\frac{1}{8} \operatorname{Tr}\left(\nabla_{\mu} \mathcal{S} \nabla_{\nu} \mathcal{S}\right) \\
\delta \mathcal{S}=-\frac{1}{2}\left(\square \mathcal{S}-\nabla_{\mu} \Phi \nabla^{\mu} \mathcal{S}\right)+\frac{1}{2} \mathcal{S} \nabla_{\mu} \mathcal{S} \nabla^{\mu} \mathcal{S}
\end{array}\right.
$$

in the convention of Eq. (2.15), to bring the result into the equivalent form

$$
I_{1}=\frac{1}{4} \alpha^{\prime} \int \mathrm{d}^{D} x \sqrt{-g} e^{-\Phi}\left[\frac{1}{16} \operatorname{Tr}\left(\nabla_{\mu} \mathcal{S} \nabla_{\nu} \mathcal{S} \nabla^{\mu} \mathcal{S} \nabla^{\nu} \mathcal{S}\right)-\frac{1}{32} \operatorname{Tr}\left(\nabla_{\mu} \mathcal{S} \nabla_{\nu} \mathcal{S}\right) \operatorname{Tr}\left(\nabla^{\mu} \mathcal{S} \nabla^{\nu} \mathcal{S}\right)\right]
$$

This precisely coincides with the truncation of Eq. (5.2) to the scalar fields. Our result reproduces also the first-order $\alpha^{\prime}$ expressions of Refs. [5,10] for the reduction to $D=1$ dimensions.

Let us finally point out that considering the most generic manifestly diffeomorphism invariant four-derivative $\operatorname{action}^{7}$ [30]

$$
\begin{aligned}
& I_{1}=\alpha^{\prime} \int \mathrm{d}^{D+d} X \sqrt{-\hat{g}} e^{-\hat{\phi}}\left(\gamma_{1} \hat{R}_{\hat{\mu} \hat{\nu} \hat{\rho} \hat{\sigma}} \hat{R}^{\hat{\mu} \hat{\nu} \hat{\rho} \hat{\sigma}}+\gamma_{2} \hat{H}^{\hat{\mu} \hat{\nu} \hat{\lambda}} \hat{H}^{\hat{\rho} \hat{\sigma}} \hat{}_{\hat{\lambda}} \hat{R}_{\hat{\mu} \hat{\nu} \hat{\rho} \hat{\sigma}}+\gamma_{3} \hat{H}_{\hat{\mu} \hat{\nu} \hat{\rho}} \hat{H}_{\hat{\sigma}}^{\hat{\mu}} \hat{\lambda}^{\hat{\lambda}} \hat{H}^{\hat{\nu}} \hat{}^{\hat{\imath}} \hat{H}^{\hat{\rho}} \hat{\hat{\sigma}}\right. \\
& \left.+\gamma_{4} \hat{H}_{\hat{\mu} \hat{\nu}}^{2} \hat{H}^{2 \hat{\mu} \hat{\nu}}+\gamma_{5}\left(\hat{H}^{2}\right)^{2}+\gamma_{6} \hat{H}_{\hat{\mu} \hat{\nu}}^{2} \partial^{\hat{\mu}} \hat{\phi} \partial^{\hat{\nu}} \hat{\phi}+\gamma_{7} \hat{H}^{2} \partial_{\hat{\mu}} \hat{\phi} \partial^{\hat{\mu}} \hat{\phi}+\gamma_{8} \partial_{\hat{\mu}} \hat{\phi} \partial^{\hat{\mu}} \hat{\phi} \partial_{\hat{\nu}} \hat{\phi} \partial^{\hat{\nu}} \hat{\phi}\right),
\end{aligned}
$$

the only choice of coefficients that give rise to an $\mathrm{O}(d, d, \mathbb{R})$ invariant action after reduction on a generic $d$-dimensional torus is

$$
\gamma_{2}=-\frac{\gamma_{1}}{2}, \quad \gamma_{3}=\frac{\gamma_{1}}{24}, \quad \gamma_{4}=-\frac{\gamma_{1}}{8}, \quad \gamma_{5}=0, \quad \gamma_{6}=0, \quad \gamma_{7}=0, \quad \gamma_{8}=0
$$

corresponding to the action (4.1). Indeed, as the definition of $\Phi$ imposes

$$
\partial_{\mu} \hat{\phi}=\partial_{\mu} \Phi+\frac{1}{2} \operatorname{Tr}\left(G^{-1} \partial_{\mu} G\right)
$$

the terms proportional to $\gamma_{6}, \gamma_{7}$, and $\gamma_{8}$, respectively, in Eq. (5.25) produce terms carrying a factor $\operatorname{Tr}\left(G^{-1} \partial_{\mu} G\right)$. However, there is no $\mathrm{O}(d, d, \mathbb{R})$ invariant term in the basis (3.26) that contains such a factor, as shown in Appendix C.

\footnotetext{
${ }^{7}$ As in Eq. (3.21) above, we restrict to manifestly diffeomorphism invariant terms. The potential gravitational ChernSimons coupling which appears for the heterotic string is discussed in detail in Sec. VII below.
}

Moreover, these terms cannot cancel each other, as they come with different contraction structures. This imposes $\gamma_{6}=\gamma_{7}=\gamma_{8}=0$. The computations detailed in Secs. II and IV finally imply the remaining coefficients of Eq. (5.26). Only with this choice do the GL $(d)$ terms combine into the $\mathrm{O}(d, d, \mathbb{R})$ invariant terms of the basis (3.26). Up to field redefinition, the action (4.1) thus is the unique four-derivative correction exhibiting $\mathrm{O}(d, d, \mathbb{R})$ invariance upon dimensional reduction.

\section{FRAME FORMULATION}

In the previous section we have shown that invariance under rigid $\mathrm{O}(d, d, \mathbb{R})$ transformations requires an $\alpha^{\prime}$ deformation of the transformation rules that resembles a 
Green-Schwarz mechanism. We will now make this analogy more precise by introducing a frame formalism for which the $\mathrm{O}(d, d, \mathbb{R})$ symmetry remains undeformed, while the local frame transformations acquire $\alpha^{\prime}$-deformations. This formulation uses the standard Green-Schwarz mechanism, albeit with composite gauge fields.

We introduce a frame field $E \equiv\left(E_{M}{ }^{A}\right)$ with inverse $E^{-1} \equiv\left(E_{A}{ }^{M}\right)$ from which the scalar matrix (2.9) encoding $G$ and $B$ can be reconstructed via

$$
\mathcal{H}_{M N}=E_{M}{ }^{A} E_{N}{ }^{B} \kappa_{A B},
$$

where flat indices are split as $A=(a, \bar{a})$, and $\kappa_{A B}$ is a block-diagonal matrix with components $\kappa_{a b}$ and $\kappa_{\bar{a} \bar{b}}$. Furthermore, we constrain the frame field by demanding that the "flattened" $\mathrm{O}(d, d, \mathbb{R})$ metric is also block-diagonal according to

$$
\eta_{A B} \equiv E_{A}{ }^{M} E_{B}{ }^{N} \eta_{M N}=\left(\begin{array}{cc}
\kappa_{a b} & 0 \\
0 & -\kappa_{\bar{a} \bar{b}}
\end{array}\right),
$$

with a relative sign in the space of barred indices reflecting the signature of the $\mathrm{O}(d, d, \mathbb{R})$ metric. In this formalism $\kappa_{a b}$ and $\kappa_{\bar{a} \bar{b}}$ need not be Kronecker deltas, and in particular can be spacetime dependent, and so there is a local GL $(d) \times$ $\mathrm{GL}(d)$ frame invariance, with transformation rules

$$
\delta_{\Lambda} E_{A}{ }^{M}=\Lambda_{A}{ }^{B} E_{B}{ }^{M}, \quad \Lambda_{A}{ }^{B}=\left(\begin{array}{cc}
\Lambda_{a}^{b} & 0 \\
0 & \bar{\Lambda}_{\bar{a}}^{\bar{b}}
\end{array}\right) .
$$

We could partially gauge fix $\kappa_{A B}=\delta_{A B}$, which reduces the frame transformations to $\mathrm{SO}(d) \times \mathrm{SO}(d)$, but in the following another gauge fixing is convenient: we identify the components of $\kappa$ with the metric $G$ according to

$$
\kappa=\left(\begin{array}{cc}
2 G & 0 \\
0 & 2 G
\end{array}\right)
$$

where we used matrix notation. A frame field satisfying the constraint (6.2) and leading to the familiar form of $\mathcal{H}_{M N}$ is then given by

$$
E \equiv\left(E_{M}^{A}\right) \equiv \frac{1}{2}\left(\begin{array}{cc}
1+B G^{-1} & 1-B G^{-1} \\
G^{-1} & -G^{-1}
\end{array}\right) .
$$

In order to derive composite connections from the frame field we define the Maurer-Cartan forms

$$
\left(E^{-1} \partial_{\mu} E\right)_{A}{ }^{B} \equiv\left(\begin{array}{cc}
Q_{\mu a}{ }^{b} & P_{\mu a}{ }^{\bar{b}} \\
\bar{P}_{\mu \bar{a}}{ }^{b} & \bar{Q}_{\mu \bar{a}} \bar{b}
\end{array}\right) .
$$

From this definition one finds that under $\operatorname{GL}(d) \times \operatorname{GL}(d)$ transformations (6.3) the $P_{\mu}$ transform as tensors, and the $Q_{\mu}$ transform as connections:

$$
\delta_{\Lambda} Q_{\mu a}{ }^{b}=-D_{\mu} \Lambda_{a}{ }^{b}, \quad \delta_{\Lambda} \bar{Q}_{\mu \bar{a}}{ }^{\bar{b}}=-D_{\mu} \bar{\Lambda}_{\bar{a}}^{\bar{b}}
$$

with $D_{\mu} \Lambda_{a}{ }^{b}=\partial_{\mu} \Lambda_{a}{ }^{b}+\left[Q_{\mu}, \Lambda\right]_{a}{ }^{b}$ and a similar formula for the barred expression. We can evaluate these connections for the gauge choice (6.5),

$$
\left\{\begin{array}{l}
Q_{\mu}=-\frac{1}{2} \partial_{\mu}(G-B) G^{-1}, \\
\bar{Q}_{\mu}=-\frac{1}{2} \partial_{\mu}(G+B) G^{-1},
\end{array}\right.
$$

using again matrix notation.

Having constructed composite gauge fields from the frame field we can consider the familiar Chern-Simons 3forms built from them:

$$
\mathrm{CS}_{\mu \nu \rho}(Q) \equiv \operatorname{Tr}\left(Q_{[\mu} \partial_{\nu} Q_{\rho]}+\frac{2}{3} Q_{[\mu} Q_{\nu} Q_{\rho]}\right)
$$

These Chern-Simons forms transform under Eq. (6.7) as

$$
\delta_{\Lambda} \mathrm{CS}_{\mu \nu \rho}(Q)=\partial_{[\mu} \operatorname{Tr}\left(\partial_{\nu} \Lambda Q_{\rho]}\right),
$$

with the barred formulas being analogous. Evaluating the Chern-Simons form with Eq. (6.8) one recovers precisely the expression (5.4) encountered in the previous section, up to a global factor of 3 . Therefore, we can define a 3-form curvature with the Chern-Simons modification,

$$
\tilde{H}_{\mu \nu \rho} \equiv H_{\mu \nu \rho}-\frac{3}{2} \alpha^{\prime}\left(\mathrm{CS}_{\mu \nu \rho}(Q)-\mathrm{CS}_{\mu \nu \rho}(\bar{Q})\right),
$$

which then reproduces the term proportional to $\Omega H$ encountered in the $\mathcal{O}\left(\alpha^{\prime}\right)$ action.

We have thus succeeded to find a formulation for which the $\mathrm{O}(d, d, \mathbb{R})$ invariance is manifestly realized without deformation. Rather, the $\mathrm{GL}(d) \times \mathrm{GL}(d)$ gauge symmetry is deformed by having a 2-form transforming according to the Green-Schwarz mechanism,

$$
\delta B_{\mu \nu}=\frac{1}{2} \alpha^{\prime} \operatorname{Tr}\left(\partial_{[\mu} \Lambda Q_{\nu]}\right)-\frac{1}{2} \alpha^{\prime} \operatorname{Tr}\left(\partial_{[\mu} \bar{\Lambda} \bar{Q}_{\nu]}\right) .
$$

Performing a partial gauge fixing to $\mathrm{SO}(d) \times \mathrm{SO}(d)$, together with appropriate field redefinitions, this GreenSchwarz mechanism relates to the reduction of $\alpha^{\prime}$-deformed double field theory [22]. This formulation is related to the one of the previous section as follows: if one fully gauge fixes $\mathrm{GL}(d) \times \mathrm{GL}(d)$, the $\mathrm{O}(d, d, \mathbb{R})$ transformations acquire deformations through compensating frame transformations, and hence the singlet $B_{\mu \nu}$ starts transforming nontrivially under $\mathrm{O}(d, d, \mathbb{R})$.

Let us close this section by discussing how the $\mathbb{Z}_{2}$ invariance (5.17) of bosonic string theory is realized in this frame formulation. The $\mathbb{Z}_{2}$ acts on the frame field as 


$$
E \rightarrow Z^{T} E \tilde{Z}, \quad \tilde{Z} \equiv\left(\begin{array}{ll}
0 & 1 \\
1 & 0
\end{array}\right) .
$$

The matrix $\tilde{Z}$ exchanges the two $\operatorname{GL}(d)$ factors and hence exchanges the role of unbarred and barred indices. Indeed, under the transformation (6.13) the Maurer-Cartan forms (6.6) transform as $P_{\mu} \leftrightarrow \bar{P}_{\mu}$ and $Q_{\mu} \leftrightarrow \bar{Q}_{\mu}$, as one may verify by a quick computation and as is suggested by the explicit form (6.8). Thus, the relative sign in Eq. (6.11) implies that the total Chern-Simons form is $\mathbb{Z}_{2}$ odd, which together with $B_{\mu \nu} \rightarrow-B_{\mu \nu}$ implies $\mathbb{Z}_{2}$ invariance of the action.

\section{GRAVITATIONAL CHERN-SIMONS FORM OF THE HETEROTIC SUPERGRAVITY}

In this section, we repeat the above analysis of the firstorder $\alpha^{\prime}$-corrections for the case of the heterotic string. In the absence of the Yang-Mills field in ten dimensions, the bosonic part of the four-derivative effective action of the heterotic string takes the form [30]

$$
\begin{aligned}
& \hat{I}_{1}=\frac{1}{4} \alpha^{\prime} \int \mathrm{d}^{D+d} X \sqrt{-\hat{g}} e^{-\hat{\phi}}\left[-\hat{H}^{\hat{\mu} \hat{\nu} \hat{\rho}} \hat{\Omega}_{\hat{\mu} \hat{\nu} \hat{\rho}}^{(\hat{\omega})}\right. \\
& +\frac{1}{2}\left(\hat{R}_{\hat{\mu} \hat{\nu} \hat{\rho} \hat{\sigma}} \hat{R}^{\hat{\mu} \hat{\nu} \hat{\rho} \hat{\sigma}}-\frac{1}{2} \hat{H}^{\hat{\mu} \hat{\nu} \hat{\lambda}} \hat{H}^{\hat{\rho} \hat{\sigma}_{\hat{\lambda}}} \hat{R}_{\hat{\mu} \hat{\nu} \hat{\rho} \hat{\sigma}}\right. \\
& \left.\left.-\frac{1}{8} \hat{H}_{\hat{\mu} \hat{\nu}}^{2} \hat{H}^{2 \hat{\mu} \hat{\nu}}+\frac{1}{24} \hat{H}_{\hat{\mu} \hat{\nu} \hat{\rho}} \hat{H}^{\hat{\mu}}{ }_{\hat{\sigma}}^{\hat{\lambda}} \hat{H}_{\hat{\lambda}}^{\hat{\nu}}{ }_{\hat{\imath}} \hat{H}_{\hat{\tau}}^{\hat{\rho}} \hat{\sigma}\right)\right] \text {, }
\end{aligned}
$$

where $D+d=10$. Apart from terms proportional to the $\alpha^{\prime}$ corrections of the bosonic string (4.1), the action features the gravitational Chern-Simons form $\hat{\Omega}_{\hat{\mu} \hat{\nu} \hat{\rho}}^{(\hat{\omega})}$, defined as

$$
\hat{\Omega}_{\hat{\mu} \hat{\nu} \hat{\rho}}^{(\hat{\omega})}=\operatorname{Tr}\left(\hat{\omega}_{[\hat{\mu}} \partial_{\hat{\nu}} \hat{\omega}_{\hat{\rho}]}\right)+\frac{2}{3} \operatorname{Tr}\left(\hat{\omega}_{[\hat{\mu}} \hat{\omega}_{\hat{\nu}} \hat{\omega}_{\hat{\rho}]}\right)
$$

in terms of the spin connection

$$
\hat{\omega}_{\hat{\mu} \hat{\alpha}} \hat{\beta}=\nabla_{\hat{\mu}} \hat{e}_{\hat{\nu}} \hat{\beta} \hat{e}_{\hat{\alpha}}^{\hat{\nu}}
$$

With the $\mathrm{O}(d, d, \mathbb{R})$ invariant form of the bosonic string discussed in Secs. IV and V above, it thus remains to reduce the first term of Eq. (7.1). We follow the same systematics outlined above.

In the flat basis, after dimensional reduction, the nonvanishing components of the spin connection are given by

$$
\begin{aligned}
& \hat{\omega}_{\alpha, \beta \gamma}=\omega_{\alpha, \beta \gamma}, \\
& \hat{\omega}_{\alpha, \beta a}=\frac{1}{2} e_{\alpha}{ }^{\mu} e_{\beta}{ }^{\nu} \eta_{a b} E_{m}{ }^{b} F_{\mu \nu}^{(1) m}, \\
& \hat{\omega}_{\alpha, a b}=e_{\alpha}{ }^{\mu} \tilde{Q}_{\mu a}{ }^{c} \eta_{c b}, \\
& \hat{\omega}_{a, \alpha \beta}=-\frac{1}{2} e_{\alpha}{ }^{\mu} e_{\beta}{ }^{\nu} \eta_{a b} E_{m}{ }^{b} F_{\mu \nu}^{(1) m}, \\
& \hat{\omega}_{a, b \alpha}=e_{\alpha}{ }^{\mu} \tilde{P}_{\mu a}{ }^{c} \eta_{c b} .
\end{aligned}
$$

Here, $\tilde{P}_{\mu a}{ }^{b}$ and $\tilde{Q}_{\mu a}{ }^{b}$ are, respectively, the symmetric and antisymmetric parts of the GL $(d)$ Maurer-Cartan form $\tilde{J}_{\mu a}{ }^{b}=E_{a}{ }^{m} \partial_{\mu} E_{m}{ }^{b}=\tilde{P}_{\mu a}{ }^{b}+\tilde{Q}_{\mu a}{ }^{b}$ and verify the integrability relations

$$
\begin{aligned}
\partial_{[\mu} \tilde{J}_{\nu] a}{ }^{b} & =-\left(\tilde{J}_{[\mu} \tilde{J}_{\nu]}\right)_{a}{ }^{b} \\
& \Leftrightarrow\left\{\begin{array}{l}
\partial_{[\mu} \tilde{P}_{\nu] a}{ }^{b}=-\left(\tilde{P}_{[\mu} \tilde{Q}_{\nu]}\right)_{a}{ }^{b}-\left(\tilde{Q}_{[\mu} \tilde{P}_{\nu]}\right)_{a}{ }^{b}, \\
\partial_{[\mu} \tilde{Q}_{\nu] a}{ }^{b}=-\left(\tilde{Q}_{[\mu} \tilde{Q}_{\nu]}\right)_{a}{ }^{b}-\left(\tilde{P}_{[\mu} \tilde{P}_{\nu]}\right)_{a}{ }^{b}
\end{array}\right.
\end{aligned}
$$

Defining the low-dimensional components of $\hat{\Omega}^{(\hat{\omega})}$ in the same way as we did for $\hat{H}$ in Eq. (2.5), we obtain

$$
\begin{aligned}
\Omega_{\mu \nu \rho}^{(\hat{\omega})}= & \Omega_{\mu \nu \rho}^{(\omega)}-\frac{1}{3} \operatorname{Tr}\left(\tilde{J}_{[\mu} \tilde{J}_{\nu} \tilde{J}_{\rho]}\right)-\frac{1}{2} F_{[\mu \mid \sigma}^{(1) m} G_{m n} \nabla_{\mid \nu} F_{\rho]}^{(1) \sigma n}+\frac{1}{4} F_{[\mu \mid \sigma}^{(1) m} \nabla^{\sigma} G_{m n} F_{\mid \nu \rho]}^{(1) n} \\
& -\frac{1}{4} e_{\alpha}{ }^{\sigma} \nabla_{[\mu \mid} e_{\tau}^{\alpha} F_{\mid \nu \rho]}^{(1) m} G_{m n} F_{\sigma}^{(1) \tau n}, \\
\Omega_{\mu \nu m}^{(\hat{\omega})}= & \frac{1}{6} R_{\mu \nu \rho \sigma} F^{(1) \rho \sigma n} G_{n m}-\frac{1}{12} F_{\mu \nu}^{(1) n}\left(\nabla_{\rho} G G^{-1} \nabla^{\rho} G\right)_{n m}-\frac{1}{6} F_{[\mu}^{(1) \rho n}\left(\nabla_{\nu]} G G^{-1} \nabla_{\rho} G\right)_{n m} \\
& -\frac{1}{24} G_{m n} F^{(1) \rho \sigma n} F_{\mu \nu}^{(1) p} G_{p q} F_{\rho \sigma}^{(1) q}-\frac{1}{12} G_{m n} F^{(1) \rho \sigma n} F_{[\mu \mid \rho}^{(1) p} G_{p q} F_{\mid \nu] \sigma}^{(1) q}-\frac{1}{6} \nabla_{[\mu \mid} \nabla^{\rho} G_{m n} F_{\mid \nu] \rho}^{(1) n} \\
& +\frac{1}{6} \nabla^{\rho} G_{m n} \nabla_{[\mu} F_{\nu] \rho}^{(1) n}+\frac{1}{6} \nabla_{[\mu}\left(\nabla_{\nu]} e_{\sigma}^{\alpha} e_{\alpha}{ }^{\rho} F_{\rho}^{(1) \sigma n} G_{n m}\right), \\
\Omega_{\mu m n}^{(\hat{\omega})}= & \frac{1}{12} F_{\rho \sigma}^{(1) p} G_{p[m \mid} \nabla_{\mu}\left(F^{(1) \rho \sigma q} G_{q \mid n]}\right)-\frac{1}{6} F_{\mu \nu}^{(1) p} \nabla_{\rho} G_{p[m} G_{n] q} F^{(1) \rho \nu q} \\
& +\frac{1}{6}\left(\nabla_{\nu} G G^{-1} \nabla_{\mu} \nabla^{\nu} G\right)_{[m n]} .
\end{aligned}
$$


We can now focus on the reduction of the action. Splitting the ten-dimensional indices $\hat{\mu}$ into $(\mu, m)$, we obtain

$$
\hat{H}^{\hat{\mu} \hat{\nu} \hat{\rho}} \hat{\Omega}_{\hat{\mu} \hat{\nu} \hat{\rho}}^{(\hat{\omega})}=H^{\mu \nu \rho} \Omega_{\mu \nu \rho}^{(\hat{\omega})}+3 H^{\mu \nu m} \Omega_{\mu \nu m}^{(\hat{\omega})}+3 H^{\mu m n} \Omega_{\mu m n}^{(\hat{\omega})}+H^{m n p} \Omega_{m n p}^{(\hat{\omega})} .
$$

Using the explicit expressions of Eqs. (2.5) and (7.6), the reduced Chern-Simons form then takes the form

$$
\begin{aligned}
- & \frac{1}{4} \alpha^{\prime} \int \mathrm{d}^{D+d} X \sqrt{-\hat{g}} e^{-\hat{\phi}} \hat{H}^{\hat{\mu} \hat{\nu} \hat{\rho}} \hat{\Omega}_{\hat{\mu} \hat{\nu} \hat{\rho}}^{(\hat{\omega})} \\
\rightarrow & \frac{\alpha^{\prime}}{4} \int \mathrm{d}^{D} x \sqrt{-g} e^{-\Phi}\left[-H^{\mu \nu \rho} \Omega_{\mu \nu \rho}^{(\omega)}+\frac{1}{3} H^{\mu \nu \rho} \operatorname{Tr}\left(\tilde{J}_{\mu} \tilde{J}_{\nu} \tilde{J}_{\rho}\right)-\frac{1}{2} R^{\mu \nu \rho \sigma} F_{\mu \nu}^{(1) m} H_{\rho \sigma m}\right. \\
& -\frac{1}{4} H^{\mu \nu \rho} F_{\mu \sigma}^{(1) m} \nabla^{\sigma} G_{m n} F_{\nu \rho}^{(1) n}+\frac{1}{8} F_{\mu \nu}^{(1) m} G_{m n} F_{\rho \sigma}^{(1) n} F^{(1) \rho \sigma p} H^{\mu \nu}{ }_{p}+\frac{1}{4} F_{\mu \nu}^{(1) m} H_{\rho \sigma m} F^{(1) \mu \rho p} G_{p q} F^{(1) \nu \sigma q} \\
& -\frac{1}{4} H_{\mu \nu m}\left(\nabla_{\rho} G^{-1} \nabla^{\rho} G\right)^{m}{ }_{n} F^{(1) \mu \nu n}-\frac{1}{4} F_{\mu \nu}^{(1) m}\left(\nabla_{\rho} B G^{-1} \nabla^{\rho} G\right)_{m n} F^{(1) \mu \nu n}+\frac{1}{2} H_{\mu \nu m}\left(\nabla_{\rho} G^{-1} \nabla^{\mu} G\right)_{n}{ }_{n} F^{(1) \nu \rho n} \\
& +\frac{1}{2} F_{\mu \nu}{ }^{(1) m}\left(\nabla_{\rho} G G^{-1} \nabla^{\mu} B\right)_{m n} F^{(1) \rho \nu n}+\frac{1}{4} H^{\mu \nu \rho} e_{\alpha}{ }^{\sigma} \nabla_{\mu} e_{\tau}{ }^{\alpha} F_{\nu \rho}^{(1) m} G_{m n} F_{\sigma}^{(1) \tau n}-\frac{1}{2} H^{\mu \nu}{ }_{m} G^{m n} \nabla_{\mu}\left(\nabla_{\nu} e_{\sigma}{ }^{\alpha} e_{\alpha}{ }^{\rho} F_{\rho}^{(1) \sigma p} G_{n p}\right) \\
& +\frac{1}{2} \operatorname{Tr}\left(\nabla_{\mu} \nabla_{\nu} G \nabla^{\mu} G^{-1} \nabla^{\nu} B G^{-1}\right)-\frac{1}{2} H_{\mu \nu m}\left(G^{-1} \nabla_{\rho} G\right)^{m}{ }_{n} \nabla^{\mu} F^{(1) \nu \rho n}+\frac{1}{2} H_{\mu \nu m}\left(G^{-1} \nabla^{\mu} \nabla_{\rho} G\right)_{n}^{m} F^{(1) \nu \rho n} \\
& \left.+\frac{1}{2} H^{\mu \nu \rho} F_{\mu \sigma}^{(1) m} G_{m n} \nabla_{\nu} F_{\rho}^{(1) \sigma n}-\frac{1}{4} F_{\mu \nu}^{(1) m} \nabla_{\rho} B_{m n} \nabla^{\rho} F^{(1) \mu \nu n}\right] .
\end{aligned}
$$

Only the last six terms carry second-order derivatives. Following the systematics of Sec. IV, these terms can be transformed by means of partial integration and Bianchi identities such that all second-order derivatives appear as the leading twoderivative contribution from the field Eqs. (2.17), i.e., appear within the first column of Table I. Details are given in Appendix B 3. Specifically, the remaining second-order derivative terms combine into

$$
\begin{aligned}
& \frac{\alpha^{\prime}}{8} \int \mathrm{d}^{D} x \sqrt{-g} e^{-\Phi}\left[\nabla_{\mu} H_{m}^{\mu \nu} F_{\rho}^{(1) \sigma m} \nabla_{\nu} e_{\sigma}^{\alpha} e_{\alpha}{ }^{\rho}-\operatorname{Tr}\left(\square B G^{-1} \nabla_{\nu} G \nabla^{\nu} G^{-1}-\square G \nabla_{\nu} G^{-1} \nabla^{\nu} B G^{-1}\right)\right. \\
& -\nabla_{\mu} F^{(1) \mu \nu m}\left(\nabla^{\rho} G G^{-1}\right)_{m}{ }^{n} H_{\nu \rho n}-\frac{1}{2} H_{\mu \nu m}\left(G^{-1} \square G\right)^{m}{ }_{n} F^{(1) \mu \nu n} \\
& \left.-\frac{1}{2} H_{\mu \nu \rho} \nabla_{\sigma} F^{(1) \sigma \mu m} G_{m n} F^{(1) \nu \rho n}-\nabla_{\mu} F^{(1) \mu \nu m} \nabla^{\rho} B_{m n} F_{\nu \rho}^{(1) n}\right],
\end{aligned}
$$

and can be eliminated by field redefinitions according to the rules defined in Table I. These take the explicit form [in the convention of Eq. (2.15)]

$$
\begin{aligned}
\delta B_{\mu \nu}= & \frac{1}{8} A_{[\mu}^{(1) m} \nabla_{\nu]} e_{\sigma}^{\alpha} e_{\alpha}{ }^{\rho} G_{m n} F_{\rho}^{(1) \sigma n}+\frac{1}{8} A_{[\mu \mid m}^{(2)} \nabla^{\rho} G^{m n} H_{\mid \nu] \rho n}-\frac{1}{8} B_{m n} A_{[\mu}^{(1) n} \nabla^{\rho} G^{m p} H_{\nu] \rho p} \\
& -\frac{1}{16} A_{[\mu \mid m}^{(2)} H_{\mid \nu] \rho \sigma} F^{(1) \rho \sigma m}+\frac{1}{16} B_{m n} A_{[\mu}^{(1) n} H_{\nu] \rho \sigma} F^{(1) \rho \sigma m}-\frac{1}{8} A_{[\mu \mid m}^{(2)}\left(G^{-1} \nabla^{\rho} B\right)^{m}{ }_{n} F_{[\nu] \rho}^{(1) n} \\
& +\frac{1}{8} B_{m n} A_{[\mu \mid}^{(1) n}\left(G^{-1} \nabla^{\rho} B\right)^{m}{ }_{p} F_{\mid \nu] \rho}^{(1) p}, \\
\delta G^{m n}= & \frac{1}{4}\left(\nabla_{\mu} G^{-1} \nabla^{\mu} B G^{-1}\right)^{(m n)}-\frac{1}{8} F^{(1) \mu \nu(m} G^{n) p} H_{\mu \nu p}, \\
\delta B_{m n}= & \frac{1}{4}\left(\nabla_{\mu} G \nabla^{\mu} G^{-1} G\right)_{[m n]}, \\
\delta A_{\mu}^{(1) m}= & -\frac{1}{8} \nabla^{\nu} G^{m n} H_{\mu \nu n}+\frac{1}{16} H_{\mu \nu \rho} F^{(1) \nu \rho m}+\frac{1}{8}\left(G^{-1} \nabla^{\nu} B\right)^{m}{ }_{n} F_{\mu \nu}^{(1) n}, \\
\delta A_{\mu m}^{(2)}= & -\frac{1}{8} \nabla_{\mu} e_{\sigma}^{\alpha} e_{\alpha}{ }^{\rho} G_{m n} F_{\rho}^{(1) \sigma n}-\frac{1}{8} B_{m n} \nabla^{\nu} G^{n p} H_{\mu \nu p}+\frac{1}{16} H_{\mu \nu \rho} B_{m n} F^{(1) \nu \rho n}+\frac{1}{8}\left(B G^{-1} \nabla^{\nu} B\right)_{m n} F_{\mu \nu}^{(1) n} .
\end{aligned}
$$


After applying these field redefinitions, the resulting reduced action no longer carries any second-order derivative (except within the Riemann tensor) and turns into

$$
\begin{aligned}
- & \frac{1}{4} \alpha^{\prime} \int \mathrm{d}^{D+d} X \sqrt{-\hat{g}} e^{-\hat{\phi}} \hat{H}^{\hat{\mu} \hat{\nu} \hat{\rho}} \hat{\Omega}_{\hat{\mu} \hat{\nu} \hat{\rho}}^{(\hat{\omega})} \\
\rightarrow & \frac{\alpha^{\prime}}{4} \int \mathrm{d}^{D} x \sqrt{-g} e^{-\Phi}\left[-H^{\mu \nu \rho} \Omega_{\mu \nu \rho}^{(\omega)}+\frac{1}{3} H^{\mu \nu \rho} \operatorname{Tr}\left(\tilde{J}_{\mu} \tilde{J}_{\nu} \tilde{J}_{\rho}\right)\right. \\
& -\frac{1}{2} R^{\mu \nu \rho \sigma} F_{\mu \nu}^{(1) m} H_{\rho \sigma m}+\frac{1}{8} H^{\mu \nu \lambda} H^{\rho \sigma}{ }_{\lambda} F_{\mu \nu}^{(1) m} H_{\rho \sigma m}-\frac{1}{4} H^{\mu \nu \rho} F_{\mu \sigma}^{(1) m} \nabla^{\sigma} G_{m n} F_{\nu \rho}^{(1) n} \\
& -\frac{1}{4} H^{\mu \nu \rho} H_{\mu \sigma m} \nabla^{\sigma} G^{m n} H_{\nu \rho n}-\frac{1}{4} H^{\mu \nu \rho} F_{\mu \sigma}^{(1) m}\left(\nabla^{\sigma} B G^{-1}\right)_{m}{ }^{n} H_{\nu \rho n} \\
& +\frac{1}{4} H^{\mu \nu \rho} H_{\mu \sigma m}\left(G^{-1} \nabla^{\sigma} B\right)^{m}{ }_{n} F_{\nu \rho}^{(1) n}+\frac{1}{8} F_{\mu \nu}^{(1) m} G_{m n} F_{\rho \sigma}^{(1) n} F^{(1) \rho \sigma p} H^{\mu \nu}{ }_{p} \\
& +\frac{1}{8} H_{\mu \nu m} G^{m n} H_{\rho \sigma n} F^{(1) \rho \sigma p} H^{\mu \nu}{ }_{p}-\frac{1}{4} H_{\mu \nu m}\left(\nabla_{\rho} G^{-1} \nabla^{\rho} G\right)^{m}{ }_{n} F^{(1) \mu \nu n} \\
& -\frac{1}{4} F_{\mu \nu}^{(1) m}\left(\nabla_{\rho} B G^{-1} \nabla^{\rho} G\right)_{m n} F^{(1) \mu \nu n}-\frac{1}{4} H_{\mu \nu m}\left(\nabla_{\rho} G^{-1} \nabla^{\rho} B G^{-1}\right)^{m n} H^{\mu \nu}{ }_{n} \\
& -\frac{1}{4} H_{\mu \nu m}\left(G^{-1} \nabla_{\rho} B G^{-1} \nabla^{\rho} B\right)^{m}{ }_{n} F^{(1) \mu \nu n}-\frac{1}{2} H_{\mu \nu m}\left(G^{-1} \nabla^{\mu} B G^{-1} \nabla_{\rho} B\right)^{m}{ }_{n} F^{(1) \nu \rho n} \\
& +\frac{1}{2} F_{\mu \nu}^{(1) m}\left(\nabla^{\mu} G G^{-1} \nabla_{\rho} B\right)_{m n} F^{(1) \nu \rho n}-\frac{1}{2} F_{\mu \nu}^{(1) m}\left(\nabla^{\mu} G \nabla_{\rho} G^{-1}\right)_{m}{ }^{n} H^{\nu \rho}{ }_{n} \\
& +\frac{1}{2} H_{\mu \nu m}\left(G^{-1} \nabla^{\mu} B \nabla_{\rho} G^{-1}\right)^{m n} H^{\nu \rho}{ }_{n}-\frac{1}{2} \operatorname{Tr}\left(\nabla_{\mu} B \nabla^{\mu} G^{-1} \nabla_{\nu} G \nabla^{\nu} G^{-1}\right) \\
& \left.+\frac{1}{2} \operatorname{Tr}\left(\nabla_{\mu} B G^{-1} \nabla^{\mu} B \nabla_{\nu} G^{-1} \nabla^{\nu} B G^{-1}\right)\right] .
\end{aligned}
$$

The terms appearing in this expression can finally be compared to the $\operatorname{GL}(d)$ decompositions of the $\mathrm{O}(d, d$, $\mathbb{R})$ basis as collected in Appendix C. This allows one to recast the result into the form

$$
\begin{aligned}
- & \frac{1}{4} \alpha^{\prime} \int \mathrm{d}^{D+d} X \sqrt{-\hat{g}} e^{-\hat{\phi}} \hat{H}^{\hat{\mu} \hat{\nu} \hat{\rho}} \hat{\Omega}_{\hat{\mu} \hat{\nu} \hat{\rho}}^{(\hat{\omega})} \\
\rightarrow & \frac{\alpha^{\prime}}{4} \int \mathrm{d}{ }^{D} x \sqrt{-g} e^{-\Phi}\left[-H^{\mu \nu \rho} \Omega_{\mu \nu \rho}^{(\omega)}+\frac{1}{16} \operatorname{Tr}\left(\mathcal{S} \nabla_{\mu} \mathcal{S} \nabla^{\mu} \mathcal{S} \nabla_{\nu} \mathcal{S} \nabla^{\nu} \mathcal{S}\right)\right. \\
& +\frac{1}{16} \mathcal{F}_{\mu \nu}{ }^{M} \mathcal{F}_{\rho \sigma M} \mathcal{F}^{\mu \nu P} \mathcal{S}_{P} Q \mathcal{F}^{\rho \sigma}{ }_{Q}-\frac{1}{4} R^{\mu \nu \rho \sigma} \mathcal{F}_{\mu \nu}^{M} \mathcal{F}_{\rho \sigma M}+\frac{1}{16} H^{\mu \nu \lambda} H^{\rho \sigma}{ }_{\lambda} \mathcal{F}_{\mu \nu}^{M} \mathcal{F}_{\rho \sigma M} \\
& -\frac{1}{8} \mathcal{F}_{\mu \nu}{ }^{M}\left(\nabla_{\rho} \mathcal{S} \nabla^{\rho} \mathcal{S}\right)_{M}{ }^{N} \mathcal{F}^{\mu \nu}{ }_{N}-\frac{1}{4} \mathcal{F}_{\mu \nu}{ }^{M}\left(\nabla^{\mu} \mathcal{S} \nabla_{\rho} \mathcal{S}\right)_{M}{ }^{N} \mathcal{F}^{\nu \rho}{ }_{N} \\
& \left.-\frac{1}{4} H^{\mu \nu \rho} \mathcal{F}_{\mu \sigma}{ }^{M} \nabla^{\sigma} \mathcal{S}_{M}{ }^{N} \mathcal{F}_{\nu \rho N}+\frac{1}{3} H^{\mu \nu \rho} \operatorname{Tr}\left(\tilde{J}_{\mu} \tilde{J}_{\nu} \tilde{J}_{\rho}\right)\right]
\end{aligned}
$$

manifestly $\mathrm{O}(d, d, \mathbb{R})$ invariant, except for the last term which carries the $\mathrm{GL}(d)$ Chern-Simons form

$$
\Omega_{\mu \nu \rho}^{(\tilde{J})}=\operatorname{Tr}\left(\tilde{J}_{[\mu} \tilde{J}_{\nu} \tilde{J}_{\rho]}\right) .
$$

This form is closed by virtue of the integrability relations (7.5). It can thus locally be integrated into a WessZumino-Witten (WZW) 2-form

$$
\Omega_{\mu \nu \rho}^{(\widetilde{J})}=3 \partial_{[\mu} \theta_{\nu \rho]}^{\mathrm{WZW}},
$$

such that the last term in Eq. (7.12) can be absorbed into a field redefinition

$$
\delta B_{\mu \nu}=\frac{1}{2} \theta_{\mu \nu}^{\mathrm{WZW}} .
$$

As $\Omega_{\mu \nu \rho}^{(\tilde{J})}$ is $\mathrm{O}(d, d, \mathbb{R})$ invariant, this does not affect the behavior of $B_{\mu \nu}$ under $\mathrm{O}(d, d, \mathbb{R})$ transformations. Putting everything together, the reduced action for the bosonic part of heterotic supergravity (in the absence of the ten-dimensional Yang-Mills field) is obtained by combining Eqs. (5.2) and (7.12) into 


$$
\begin{aligned}
& I=\int \mathrm{d}^{D} x \sqrt{-g} e^{-\Phi}\left[R+\partial_{\mu} \Phi \partial^{\mu} \Phi-\frac{1}{12} \tilde{H}_{\mu \nu \rho} \tilde{H}^{\mu \nu \rho}+\frac{1}{8} \operatorname{Tr}\left(\partial_{\mu} \mathcal{S} \partial^{\mu} \mathcal{S}\right)-\frac{1}{4} \mathcal{F}_{\mu \nu}^{M} \mathcal{S}_{M}{ }^{N} \mathcal{F}_{N}^{\mu \nu}\right. \\
& -\frac{1}{4} \alpha^{\prime}\left(H^{\mu \nu \rho} \Omega_{\mu \nu \rho}^{(\omega)}-\frac{1}{16} \operatorname{Tr}\left(\mathcal{S} \nabla_{\mu} \mathcal{S} \nabla^{\mu} \mathcal{S} \nabla_{\nu} \mathcal{S} \nabla^{\nu} \mathcal{S}\right)-\frac{1}{16} \mathcal{F}_{\mu \nu}{ }^{M} \mathcal{F}_{\rho \sigma M} \mathcal{F}^{\mu \nu P} \mathcal{S}_{P}{ }^{Q} \mathcal{F}^{\rho \sigma}{ }_{Q}\right. \\
& +\frac{1}{4} R^{\mu \nu \rho \sigma} \mathcal{F}_{\mu \nu}^{M} \mathcal{F}_{\rho \sigma M}-\frac{1}{16} H^{\mu \nu \lambda} H^{\rho \sigma}{ }_{\lambda} \mathcal{F}_{\mu \nu}^{M} \mathcal{F}_{\rho \sigma M}+\frac{1}{8} \mathcal{F}_{\mu \nu}{ }^{M}\left(\nabla_{\rho} \mathcal{S} \nabla^{\rho} \mathcal{S}\right)_{M}{ }^{N} \mathcal{F}^{\mu \nu}{ }_{N} \\
& \left.+\frac{1}{4} \mathcal{F}_{\mu \nu}{ }^{M}\left(\nabla^{\mu} \mathcal{S} \nabla_{\rho} \mathcal{S}\right)_{M}{ }^{N} \mathcal{F}^{\nu \rho}{ }_{N}+\frac{1}{4} H^{\mu \nu \rho} \mathcal{F}_{\mu \sigma}{ }^{M} \nabla^{\sigma} \mathcal{S}_{M}{ }^{N} \mathcal{F}_{\nu \rho N}\right) \\
& +\frac{1}{8} \alpha^{\prime}\left(R_{\mu \nu \rho \sigma} R^{\mu \nu \rho \sigma}-\frac{1}{2} R_{\mu \nu \rho \sigma} H^{\mu \nu \lambda} H_{\lambda}^{\rho \sigma}+\frac{1}{24} H_{\mu \nu \rho} H_{\sigma}^{\mu}{ }^{\lambda} H^{\nu}{ }_{\lambda}^{\tau} H_{\tau}^{\rho}{ }_{\tau}^{\sigma}\right. \\
& -\frac{1}{8} H_{\mu \nu}^{2} H^{2 \mu \nu}+\frac{1}{16} \operatorname{Tr}\left(\nabla_{\mu} \mathcal{S} \nabla_{\nu} \mathcal{S} \nabla^{\mu} \mathcal{S} \nabla^{\nu} \mathcal{S}\right)-\frac{1}{32} \operatorname{Tr}\left(\nabla_{\mu} \mathcal{S} \nabla_{\nu} \mathcal{S}\right) \operatorname{Tr}\left(\nabla^{\mu} \mathcal{S} \nabla^{\nu} \mathcal{S}\right) \\
& +\frac{1}{8} \mathcal{F}_{\mu \nu}{ }^{M} \mathcal{S}_{M}{ }^{N} \mathcal{F}_{\rho \sigma N} \mathcal{F}^{\mu \rho P} \mathcal{S}_{P}{ }^{Q} \mathcal{F}^{\nu \sigma}{ }_{Q}-\frac{1}{2} \mathcal{F}_{\mu \nu}{ }^{M} \mathcal{S}_{M}{ }^{N} \mathcal{F}^{\mu \rho}{ }_{N} \mathcal{F}^{\nu \sigma P} \mathcal{S}_{P}{ }^{Q} \mathcal{F}_{\rho \sigma Q} \\
& +\frac{1}{8} \mathcal{F}_{\mu \nu}{ }^{M} \mathcal{F}_{\rho \sigma} \mathcal{F}^{\mu \rho N} \mathcal{F}^{\nu \sigma}{ }_{N}-\frac{1}{2} R_{\mu \nu \rho \sigma} \mathcal{F}^{\mu \nu M} \mathcal{S}_{M}{ }^{N} \mathcal{F}^{\rho \sigma}{ }_{N} \\
& +\frac{1}{8} H_{\mu \nu}^{2} \operatorname{Tr}\left(\nabla^{\mu} \mathcal{S} \nabla^{\nu} \mathcal{S}\right)-\frac{1}{2} H_{\mu \nu}^{2} \mathcal{F}_{\rho}^{\mu}{ }_{\rho}^{M} \mathcal{S}_{M}{ }^{N} \mathcal{F}^{\nu \rho}{ }_{N}+\frac{1}{4} H^{\mu \nu \lambda} H^{\rho \sigma}{ }_{\lambda} \mathcal{F}_{\mu \rho}{ }^{M} \mathcal{S}_{M}{ }^{N} \mathcal{F}_{\nu \sigma N} \\
& -\frac{1}{2} \mathcal{F}_{\mu \nu}{ }^{M}\left(\mathcal{S} \nabla_{\rho} \mathcal{S} \nabla^{\nu} \mathcal{S}\right)_{M}{ }^{N} \mathcal{F}^{\mu \rho}{ }_{N}+\frac{1}{4} \mathcal{F}^{\mu \rho M} \mathcal{S}_{M}{ }^{N} \mathcal{F}^{\nu}{ }_{\rho N} \operatorname{Tr}\left(\nabla_{\mu} \mathcal{S} \nabla_{\nu} \mathcal{S}\right) \\
& \left.\left.-\frac{1}{2} H^{\mu \nu \rho} \mathcal{F}_{\mu \sigma}{ }^{M}\left(\mathcal{S} \nabla_{\nu} \mathcal{S}\right)_{M}{ }^{N} \mathcal{F}_{\rho}{ }^{\sigma}{ }{ }^{\prime}\right)\right] .
\end{aligned}
$$

Let us finally note that one could have started equivalently from the ten-dimensional action formulated in terms of the gravitational Chern-Simons form built from the Christoffel connection

$$
\hat{\Omega}_{\hat{\mu} \hat{\nu} \hat{\rho}}^{(\hat{\Gamma})}=\hat{\Gamma}_{[\hat{\mu} \mid \hat{\sigma}}^{\hat{\tau}} \partial_{\mid \hat{\nu}} \hat{\Gamma}_{\hat{\rho}] \hat{\tau}}^{\hat{\sigma}}+\frac{2}{3} \hat{\Gamma}_{[\hat{\mu} \mid \hat{\sigma}}^{\hat{\tau}} \hat{\Gamma}_{|\hat{\nu}| \hat{\eta}}^{\hat{\tau}} \hat{\Gamma}_{\mid \hat{\rho}] \hat{\sigma}}^{\hat{\eta}}
$$

This form is invariant under Lorentz transformations and related to Eq. (7.2) by [17]

$$
\begin{aligned}
\hat{\Omega}_{\hat{\mu} \hat{\nu} \hat{\rho}}^{(\hat{\Gamma})}= & \hat{\Omega}_{\hat{\mu} \hat{\nu} \hat{\rho}}^{(\hat{\rho})}+\partial_{[\hat{\mu}}\left(\partial_{\hat{\nu} \mid} \hat{e}_{\hat{\sigma}}^{\hat{\alpha}} \hat{e}_{\hat{\beta}}^{\hat{\sigma}} \hat{\omega}_{\mid \hat{\rho}] \hat{\alpha}}^{\hat{\beta}}\right) \\
& +\frac{1}{3} \operatorname{Tr}\left(\partial_{[\hat{\mu}} \hat{e} \hat{e}^{-1} \partial_{\hat{\nu}} \hat{e} \hat{e}^{-1} \partial_{\hat{\rho}]} \hat{e} \hat{e}^{-1}\right),
\end{aligned}
$$

with the difference given by two closed terms that can be absorbed by a ten-dimensional field redefinition. Dimensional reduction of the resulting ten-dimensional action then induces a lower-dimensional action in which the $\operatorname{Tr}\left(\tilde{J}^{3}\right)$ term from Eq. (7.12) is no longer present. The field redefinition required in order to absorb the closed terms of Eq. (7.18) precisely corresponds to the lower-dimensional field redefinition we have encountered in Eq. (7.15).

\section{CONCLUSIONS}

In this paper we have set up a systematic procedure for analyzing the higher-derivative corrections of the bosonic and the heterotic string upon toroidal compactification. In particular, we have discussed how to control the ambiguities that arise due to nonlinear field redefinitions and partial integration. This establishes the basis for analyzing the realization of $\mathrm{O}(d, d, \mathbb{R})$ invariance of the dimensionally reduced action. At first order in $\alpha^{\prime}$, we have presented the explicit reduction of the bosonic string and cast the result into a manifestly $\mathrm{O}(d, d, \mathbb{R})$ invariant form upon identification of the necessary field redefinitions. In particular, the analysis confirms that at order $\alpha^{\prime}$, the $\mathrm{O}(d, d, \mathbb{R})$ invariance of the dimensionally reduced action fixes all the couplings in higher dimensions (up to an overall factor). The analysis has revealed the need for a Green-Schwarz type mechanism by which the lower-dimensional 2-form [which is originally singlet under $\mathrm{O}(d, d, \mathbb{R})]$ acquires a nontrivial transformation of order $\alpha^{\prime}$. This is a genuine deformation which cannot be eliminated by further field redefinitions.

We have also extended the analysis to the bosonic sector of the heterotic string (in the absence of the ten-dimensional vector fields). In particular, we have given the complete set of nonlinear field redefinitions (4.15) and (7.10) which translate between the original ten-dimensional fields and the $\mathrm{O}(d, d, \mathbb{R})$-covariant lower-dimensional fields. This dictionary allows one to exploit the $\mathrm{O}(d, d, \mathbb{R})$ symmetry as a solution generating method for the heterotic string $[34,35]$ to first order in $\alpha^{\prime}$. Examples of such solutions have been 
constructed in Refs. [36-38]. It would be very interesting to extend the analysis to also include the ten-dimensional vector fields [39], resulting in an $\mathrm{O}(d, d+K, \mathbb{R})$ extension of the present results with the larger group broken down by the non-Abelian gauge couplings [27].

In principle, the method we have outlined is fully systematic and could be applied to higher-order $\alpha^{\prime}$-corrections. In practice, the number of terms quickly explodes and calls for complementary techniques to be combined with the present approach. As noted above, already at order $\alpha^{2}$ the number of manifestly $\mathrm{O}(d, d, \mathbb{R})$ invariant terms in lower dimensions amounts to 1817. Nevertheless, it would be interesting to compare the resulting structures to related work in Refs. [40,41]. It would also be interesting to investigate the effect of $\alpha^{\prime}$-corrections on the more general Yang-Baxter type deformations recently explored in Ref. [42].

Finally, it will be interesting to further study the simplifications arising in the resulting actions upon reduction to particularly low dimensions $D$. For $D=1$, all terms other than the scalar couplings disappear from Eqs. (5.20) and (7.16), and we recover the lowest-order result of Refs. [5,10,11]. At $D=2$, the 3 -form couplings disappear and the vector fields may be integrated out. Particularly interesting is the three-dimensional case. At $D=3$, the 2 -form may be integrated out. With a field equation of the type

$$
\nabla^{\mu}\left(e^{-\Phi} H_{\mu \nu \rho}\right)=\mathcal{O}\left(\alpha^{\prime}\right),
$$

this introduces an integration constant which in particular turns the coupling (5.4) into a three-dimensional analogue of the Wess-Zumino-Witten model (cf. Ref. [32]). Furthermore, in $D=3$, the (Abelian) vector fields may be dualized into scalars. While this dualization is still possible in the presence of $\alpha^{\prime}$-corrections, the symmetry enhancement to $\mathrm{O}(d+1, d+1, \mathbb{R})$ encountered for the two-derivative action breaks down at order $\alpha^{\prime}$ and is replaced by the appearance of the relevant automorphic forms $[43,44]$.

\section{ACKNOWLEDGMENTS}

We thank J. Maharana and D. Marqués for helpful discussions. The work of O. H. is supported by the ERC Consolidator Grant "Symmetries \& Cosmology."

\section{APPENDIX A: BASIS AT ORDER $\alpha^{\prime}$}

In this Appendix, we explicitly spell out the $\mathrm{O}(d, d, \mathbb{R})$ invariant basis schematically given in Eq. (3.26), whose existence we have deduced in Sec. III D and which we have used in order to bring the reduced action into manifestly $\mathrm{O}(d, d, \mathbb{R})$ invariant form. The basis is built from 61 terms which we list according to their different structures.

$R^{2}$

$$
\left\{R_{\mu \nu \rho \sigma} R^{\mu \nu \rho \sigma}\right\}
$$

$H^{4}$ :

$$
\left\{\left(H^{2}\right)^{2}, H^{2 \mu \nu} H_{\mu \nu}^{2}, H_{\mu \nu \rho} H^{\mu}{ }_{\alpha}^{\beta} H^{\nu}{ }_{\beta}^{\gamma} H^{\rho}{ }_{\gamma}{ }^{\alpha}\right\}
$$

$(\nabla \Phi)^{4}:$

$$
\left\{\nabla_{\mu} \Phi \nabla^{\mu} \Phi \nabla_{\nu} \Phi \nabla^{\nu} \Phi\right\}
$$

$(\nabla \mathcal{S})^{4}:$

$$
\begin{aligned}
& \left\{\operatorname{Tr}\left(\nabla_{\mu} \mathcal{S} \nabla^{\mu} \mathcal{S} \nabla_{\nu} \mathcal{S} \nabla^{\nu} \mathcal{S}\right), \operatorname{Tr}\left(\nabla_{\mu} \mathcal{S} \nabla_{\nu} \mathcal{S} \nabla^{\mu} \mathcal{S} \nabla^{\nu} \mathcal{S}\right), \operatorname{Tr}\left(\mathcal{S} \nabla_{\mu} \mathcal{S} \nabla^{\mu} \mathcal{S} \nabla_{\nu} \mathcal{S} \nabla^{\nu} \mathcal{S}\right)\right. \\
& \left.\operatorname{Tr}\left(\nabla_{\mu} \mathcal{S} \nabla^{\mu} \mathcal{S}\right) \operatorname{Tr}\left(\nabla_{\nu} \mathcal{S} \nabla^{\nu} \mathcal{S}\right), \operatorname{Tr}\left(\nabla_{\mu} \mathcal{S} \nabla_{\nu} \mathcal{S}\right) \operatorname{Tr}\left(\nabla^{\mu} \mathcal{S} \nabla^{\nu} \mathcal{S}\right)\right\}
\end{aligned}
$$

$\mathcal{F}^{4}$ :

$$
\begin{aligned}
& \left\{\mathcal{F}_{\mu \nu}{ }^{M} \mathcal{F}^{\mu \nu}{ }_{M} \mathcal{F}_{\rho \sigma}{ }^{N} \mathcal{F}^{\rho \sigma}{ }_{N}, \mathcal{F}_{\mu \nu}{ }^{M} \mathcal{S}_{M}{ }^{N} \mathcal{F}^{\mu \nu}{ }_{N} \mathcal{F}_{\rho \sigma}{ }^{P} \mathcal{F}^{\rho \sigma}{ }_{P}, \mathcal{F}_{\mu \nu}{ }^{M} \mathcal{S}_{M}{ }^{N} \mathcal{F}^{\mu \nu}{ }_{N} \mathcal{F}_{\rho \sigma}{ }^{P} \mathcal{S}_{P} Q \mathcal{F}^{\rho \sigma}{ }_{Q},\right. \\
& \mathcal{F}_{\mu \nu}{ }^{M} \mathcal{F}_{\rho \sigma} \mathcal{F}^{\mu \nu N} \mathcal{F}^{\rho \sigma}{ }_{N}, \mathcal{F}_{\mu \nu}{ }^{M} \mathcal{S}_{M}{ }^{N} \mathcal{F}_{\rho \sigma}{ }_{\rho} \mathcal{F}^{\mu \nu} P \mathcal{F}^{\rho \sigma}{ }_{P}, \mathcal{F}_{\mu \nu}{ }^{M} \mathcal{S}_{M}{ }^{N} \mathcal{F}_{\rho \sigma}{ }_{\rho} \mathcal{F}^{\mu \nu P} \mathcal{S}_{P} Q \mathcal{F}^{\rho \sigma}{ }_{Q}, \\
& \mathcal{F}_{\mu \nu}{ }^{M} \mathcal{F}_{\rho \sigma_{M}} \mathcal{F}^{\mu \rho N} \mathcal{F}^{\nu \sigma}{ }_{N}, \mathcal{F}_{\mu \nu}{ }^{M} \mathcal{S}_{M}{ }^{N} \mathcal{F}_{\rho \sigma_{N}} \mathcal{F}^{\mu \rho P} \mathcal{F}^{\nu \sigma}{ }_{P}, \mathcal{F}_{\mu \nu}{ }^{M} \mathcal{S}_{M}{ }^{N} \mathcal{F}_{\rho \sigma_{N}} \mathcal{F}^{\mu \rho P} \mathcal{S}_{P} Q \mathcal{F}^{\nu \sigma}{ }_{Q}, \\
& \left.\mathcal{F}_{\mu \nu}{ }^{M} \mathcal{F}^{\mu \rho}{ }_{M} \mathcal{F}^{\nu \sigma N} \mathcal{F}_{\rho \sigma}, \mathcal{F}_{\mu \nu}{ }^{M} \mathcal{S}_{M}{ }^{N} \mathcal{F}^{\mu \rho}{ }_{N} \mathcal{F}^{\nu \sigma P} \mathcal{F}_{\rho \sigma P}, \mathcal{F}_{\mu \nu}{ }^{M} \mathcal{S}_{M}{ }^{N} \mathcal{F}^{\mu \rho}{ }_{N} \mathcal{F}^{\nu \sigma P} \mathcal{S}_{P} Q \mathcal{F}_{\rho \sigma}{ }\right\}
\end{aligned}
$$


$R H^{2}:$

$$
\left\{R_{\mu \nu \rho \sigma} H^{\mu \nu \lambda} H_{\lambda}^{\rho \sigma}\right\}
$$

$R \mathcal{F}^{2}$ :

$$
\left\{R_{\mu \nu \rho \sigma} \mathcal{F}^{\mu \nu M} \mathcal{F}^{\rho \sigma}{ }_{M}, R_{\mu \nu \rho \sigma} \mathcal{F}^{\mu \nu M} \mathcal{S}_{M}{ }^{N} \mathcal{F}^{\rho \sigma}{ }_{N}\right\}
$$

$H^{2}(\nabla \Phi)^{2}$

$$
\left\{H_{\mu \nu}^{2} \nabla^{\mu} \Phi \nabla^{\nu} \Phi, H^{2} \nabla_{\mu} \Phi \nabla^{\mu} \Phi\right\}
$$

$H^{2}(\nabla \mathcal{S})^{2}$ :

$$
\left\{H_{\mu \nu}^{2} \operatorname{Tr}\left(\nabla^{\mu} \mathcal{S} \nabla^{\nu} \mathcal{S}\right), H^{2} \operatorname{Tr}\left(\nabla_{\mu} \mathcal{S} \nabla^{\mu} \mathcal{S}\right)\right\}
$$

$H^{2} \mathcal{F}^{2}$ :

$$
\begin{aligned}
& \left\{H^{2} \mathcal{F}_{\mu \nu}{ }^{M} \mathcal{F}^{\mu \nu}{ }_{M}, H^{2} \mathcal{F}_{\mu \nu}{ }^{M} \mathcal{S}_{M}{ }^{N} \mathcal{F}^{\mu \nu}{ }_{N}, H_{\mu \nu}^{2} \mathcal{F}^{\mu \rho M} \mathcal{F}_{\rho}{ }^{\nu}{ }_{M}, H_{\mu \nu}{ }_{\mu \nu} \mathcal{F}^{\mu \rho M} \mathcal{S}_{M}{ }^{N} \mathcal{F}_{\rho}{ }^{\nu}{ }_{N},\right. \\
& H_{\mu \nu}{ }^{\lambda} H_{\lambda \rho \sigma} \mathcal{F}^{\mu \nu M} \mathcal{F}^{\rho \sigma}{ }_{M}, H_{\mu \nu}{ }^{\lambda} H_{\lambda \rho \sigma} \mathcal{F}^{\mu \nu M} \mathcal{S}_{M}{ }^{N} \mathcal{F}^{\rho \sigma}{ }_{N}, H_{\mu \nu}{ }^{\lambda} H_{\lambda \rho \sigma} \mathcal{F}^{\mu \sigma M} \mathcal{F}^{\rho \nu}{ }_{M}, \\
& \left.H_{\mu \nu}{ }^{\lambda} H_{\lambda \rho \sigma} \mathcal{F}^{\mu \sigma M} \mathcal{S}_{M}{ }^{N} \mathcal{F}^{\rho \nu}{ }_{N}\right\}
\end{aligned}
$$

$(\nabla \Phi)^{2}(\nabla \mathcal{S})^{2}$

$$
\left\{\nabla_{\mu} \Phi \nabla_{\nu} \Phi \operatorname{Tr}\left(\nabla^{\mu} \mathcal{S} \nabla^{\nu} \mathcal{S}\right), \nabla_{\mu} \Phi \nabla^{\mu} \Phi \operatorname{Tr}\left(\nabla_{\nu} \mathcal{S} \nabla^{\nu} \mathcal{S}\right)\right\}
$$

$(\nabla \Phi)^{2} \mathcal{F}^{2}$

$$
\begin{gathered}
\left\{\nabla_{\rho} \Phi \nabla^{\rho} \Phi \mathcal{F}_{\mu \nu}{ }^{M} \mathcal{F}^{\mu \nu}{ }_{M}, \nabla_{\rho} \Phi \nabla^{\rho} \Phi \mathcal{F}_{\mu \nu}{ }^{M} \mathcal{S}_{M}{ }^{N} \mathcal{F}^{\mu \nu}{ }_{N},\right. \\
\left.\nabla^{\mu} \Phi \nabla_{\nu} \Phi \mathcal{F}_{\rho \mu}{ }^{M} \mathcal{F}^{\rho \nu}{ }_{M}, \nabla^{\mu} \Phi \nabla_{\nu} \Phi \mathcal{F}_{\rho \mu}{ }^{M} \mathcal{S}_{M}{ }^{N} \mathcal{F}^{\rho \nu}{ }_{N}\right\}
\end{gathered}
$$

$(\nabla \mathcal{S})^{2} \mathcal{F}^{2}$

$$
\begin{gathered}
\left\{\operatorname{Tr}\left(\nabla_{\rho} \mathcal{S} \nabla^{\rho} \mathcal{S}\right) \mathcal{F}_{\mu \nu}{ }^{M} \mathcal{F}^{\mu \nu}{ }_{M}, \operatorname{Tr}\left(\nabla_{\rho} \mathcal{S} \nabla^{\rho} \mathcal{S}\right) \mathcal{F}_{\mu \nu}{ }^{M} \mathcal{S}_{M}{ }^{N} \mathcal{F}^{\mu \nu}{ }_{N},\right. \\
\operatorname{Tr}\left(\nabla^{\mu} \mathcal{S} \nabla^{\nu} \mathcal{S}\right) \mathcal{F}_{\mu \rho}{ }^{M} \mathcal{F}_{\nu}{ }^{\rho}{ }_{M}, \operatorname{Tr}\left(\nabla^{\mu} \mathcal{S} \nabla^{\nu} \mathcal{S}\right) \mathcal{F}_{\mu \rho}{ }^{M} \mathcal{S}_{M}{ }^{N} \mathcal{F}_{\nu}{ }{ }_{N}, \\
\mathcal{F}_{\mu \nu}{ }^{M} \nabla_{\rho} \mathcal{S}_{M}{ }^{N} \nabla^{\rho} \mathcal{S}_{N}{ }^{P} \mathcal{F}^{\mu \nu}{ }_{P}, \mathcal{F}_{\mu \nu}{ }^{M} \nabla_{\rho} \mathcal{S}_{M}{ }^{N} \nabla^{\rho} \mathcal{S}_{N}{ }^{P} \mathcal{S}_{P}{ }^{Q} \mathcal{F}^{\mu \nu}{ }_{Q}, \\
\mathcal{F}_{\mu \nu}{ }^{M} \nabla^{\nu} \mathcal{S}_{M}{ }^{N} \nabla_{\rho} \mathcal{S}_{N}{ }^{P} \mathcal{F}^{\mu \rho}{ }_{P}, \mathcal{F}_{\mu \nu}{ }^{M} \nabla^{\nu} \mathcal{S}_{M}{ }^{N} \nabla_{\rho} \mathcal{S}_{N}{ }^{P} \mathcal{S}_{P}{ }^{Q} \mathcal{F}^{\mu \rho}{ }_{Q}, \\
\left.\mathcal{F}_{\mu \nu}{ }^{M} \nabla_{\rho} \mathcal{S}_{M}{ }^{N} \nabla^{\nu} \mathcal{S}_{N}{ }^{P} \mathcal{F}^{\mu \rho}{ }_{P}, \mathcal{F}_{\mu \nu}{ }^{M} \nabla_{\rho} \mathcal{S}_{M}{ }^{N} \nabla^{\nu} \mathcal{S}_{N}{ }^{P} \mathcal{S}_{P}{ }^{Q} \mathcal{F}^{\mu \rho}{ }_{Q}\right\}
\end{gathered}
$$

$H \nabla \Phi \mathcal{F}^{2}$ :

$$
\left\{H^{\mu \nu \rho} \nabla^{\sigma} \Phi \mathcal{F}_{\mu \nu}{ }^{M} \mathcal{F}_{\rho \sigma_{M}}, H^{\mu \nu \rho} \nabla^{\sigma} \Phi \mathcal{F}_{\mu \nu}{ }^{M} \mathcal{S}_{M}{ }^{N} \mathcal{F}_{\rho \sigma_{N}}\right\}
$$

$H \nabla \mathcal{S} \mathcal{F}^{2}:$

$$
\left\{H^{\mu \nu \rho} \mathcal{F}_{\mu \sigma}{ }^{M} \nabla_{\nu} \mathcal{S}_{M}{ }^{N} \mathcal{S}_{N}{ }^{P} \mathcal{F}_{\rho}{ }^{\sigma}{ }_{P}, H^{\mu \nu \rho} \mathcal{F}_{\mu \nu}{ }^{M} \nabla^{\sigma} \mathcal{S}_{M}{ }^{N} \mathcal{F}_{\rho \sigma_{N}}, H^{\mu \nu \rho} \mathcal{F}_{\mu \nu}{ }^{M} \nabla^{\sigma} \mathcal{S}_{M}{ }^{N} \mathcal{S}_{N}{ }^{P} \mathcal{F}_{\rho \sigma P}\right\}
$$

$\nabla \Phi \nabla \mathcal{S} \mathcal{F}^{2}:$

$$
\left\{\nabla^{\rho} \Phi \mathcal{F}_{\mu \nu}{ }^{M} \nabla_{\rho} \mathcal{S}_{M}{ }^{N} \mathcal{F}^{\mu \nu}{ }_{N}, \nabla^{\mu} \Phi \mathcal{F}_{\mu \rho}{ }^{M} \nabla_{\nu} \mathcal{S}_{M}{ }^{N} \mathcal{F}^{\nu \rho}{ }_{N}, \nabla^{\mu} \Phi \mathcal{F}_{\mu \rho}{ }^{M} \nabla_{\nu} \mathcal{S}_{M}{ }^{N} \mathcal{S}_{N}{ }^{P} \mathcal{F}^{\nu \rho}{ }_{P}\right\}
$$




\section{APPENDIX B: PARTIAL INTEGRATION AND EXPLICIT FIELD REDEFINITIONS}

In this Appendix, we give some details about the computations of the dimensionally reduced actions presented in Sec. IV A, and Sec. VII, respectively. We show explicitly how to eliminate all second-order derivatives by partial integration up to terms appearing in the first column of Table I, amenable to subsequent elimination by field redefinitions.

\section{1. $\hat{\boldsymbol{R}}_{\hat{\mu} \hat{\nu} \hat{\rho} \hat{\sigma}} \hat{\boldsymbol{R}}^{\hat{\mu} \hat{\nu} \hat{\rho} \hat{\sigma}}$}

Let us begin with the terms appearing in the reduction of $\hat{R}_{\hat{\mu} \hat{\nu} \hat{\rho} \hat{\sigma}} \hat{R}^{\hat{\mu} \hat{\nu} \hat{\rho} \hat{\sigma}}$, as presented in Sec. IVA. We give the explicit expression of the five last terms in Eq. (4.8) after integration by parts (and use of Bianchi identities). Up to boundary terms (which we ignore), the first two terms can be rewritten as

$$
\begin{aligned}
& \frac{\alpha^{\prime}}{4} \int \mathrm{d}^{D} x \sqrt{-g} e^{-\Phi} \operatorname{Tr}\left(\nabla_{\mu} \nabla_{\nu} G^{-1} G \nabla^{\mu} \nabla^{\nu} G^{-1} G\right) \\
& =\frac{\alpha^{\prime}}{4} \int \mathrm{d}^{D} x \sqrt{-g} e^{-\Phi}\left[\operatorname{Tr}\left(\left(\square G^{-1}-\nabla_{\mu} \Phi \nabla^{\mu} G^{-1}\right) G\left(\square G^{-1}-\nabla_{\nu} \Phi \nabla^{\nu} G^{-1}\right) G\right)\right. \\
& \quad+2 \operatorname{Tr}\left(\left(\square G^{-1}-\nabla_{\mu} \Phi \nabla^{\mu} G^{-1}\right) G \nabla_{\nu} G^{-1} \nabla^{\nu} G\right)-\operatorname{Tr}\left(\left(\square G-\nabla_{\mu} \Phi \nabla^{\mu} G\right) G^{-1} \nabla_{\nu} G \nabla^{\nu} G^{-1}\right) \\
& \left.\quad+\operatorname{Tr}\left(\nabla_{\mu} G^{-1} \nabla_{\nu} G \nabla^{\mu} G^{-1} \nabla^{\nu} G\right)+\left(R_{\mu \nu}+\nabla_{\mu} \nabla_{\nu} \Phi\right) \operatorname{Tr}\left(\nabla^{\mu} G^{-1} \nabla^{\nu} G\right)\right]
\end{aligned}
$$

and

$$
\begin{aligned}
& \frac{3 \alpha^{\prime}}{4} \int \mathrm{d}^{D} x \sqrt{-g} e^{-\Phi} \operatorname{Tr}\left(\nabla_{\mu} \nabla_{\nu} G^{-1} \nabla^{\mu} G \nabla^{\nu} G^{-1} G\right) \\
& =\int \mathrm{d}^{D} x \sqrt{-g} e^{-\Phi} \frac{\alpha^{\prime}}{4}\left[\frac{3}{2} \operatorname{Tr}\left(\left(\square G-\nabla_{\mu} \Phi \nabla^{\mu} G\right) G^{-1} \nabla_{\nu} G \nabla^{\nu} G^{-1}\right)-\frac{3}{2} \operatorname{Tr}\left(\nabla_{\mu} G^{-1} \nabla_{\nu} G \nabla^{\mu} G^{-1} \nabla^{\nu} G\right)\right],
\end{aligned}
$$

respectively. The last three terms can be manipulated similarly and their sum takes the following form:

$$
\begin{aligned}
& \frac{\alpha^{\prime}}{4} \int \mathrm{d}^{D} x \sqrt{-g} e^{-\Phi}\left[F_{\mu \nu}^{(1) m}\left(G \nabla^{\mu} \nabla_{\rho} G^{-1} G\right)_{m n} F^{(1) \rho \nu n}-2 \nabla_{\rho} F_{\mu \nu}^{(1) m} G_{m n} \nabla^{\mu} F^{(1) \nu \rho n}-6 \nabla_{\rho} F_{\mu \nu}^{(1) m} \nabla^{\mu} G_{m n} F^{(1) \nu \rho n}\right] \\
& =\int \mathrm{d}^{D} x \sqrt{-g} e^{-\Phi} \frac{\alpha^{\prime}}{4}\left[2\left(\nabla^{\mu} F_{\mu \nu}^{(1) m}-\nabla^{\mu} \Phi F_{\mu \nu}^{(1) m}\right) G_{m n}\left(\nabla_{\rho} F^{(1) \rho \nu n}-\nabla_{\rho} \Phi F^{(1) \rho \nu n}\right)\right. \\
& -2\left(R^{\mu \nu}+\nabla^{\mu} \nabla^{\nu} \Phi\right) F_{\mu \rho}^{(1) m} G_{m n} F_{\nu}^{(1) \rho n}-\frac{5}{4} F_{\mu \nu}^{(1) m}\left(\square G-\nabla_{\rho} \Phi \nabla^{\rho} G\right)_{m n} F^{(1) \mu \nu n} \\
& +F_{\mu \nu}^{(1) m}\left(\nabla_{\rho} G \nabla^{\mu} G^{-1} G\right)_{m n} F^{(1) \nu \rho n}+F_{\mu \nu}^{(1) m}\left(\nabla^{\mu} G \nabla_{\rho} G^{-1} G\right)_{m n} F^{(1) \nu \rho n} \\
& \left.+3 F_{\mu \nu}^{(1) m} \nabla^{\mu} G_{m n}\left(\nabla_{\rho} F^{(1) \rho \nu n}-\nabla_{\rho} \Phi F^{(1) \rho \nu n}\right)+R^{\mu \nu \rho \sigma} F_{\mu \nu}^{(1) m} G_{m n} F_{\rho \sigma}^{(1) n}\right]
\end{aligned}
$$

again up to boundary contributions. In the form (B1)-(B3), all the remaining second-order derivatives are of the form appearing in the first column of Table I. They can thus be reabsorbed into field redefinitions as discussed in Sec. II B. Explicitly, this induces the order $\alpha^{\prime}$ field redefinitions

$$
\begin{aligned}
\delta \Phi= & \frac{1}{8}\left[-2 F_{\mu \nu}^{(1) m} G_{m n} F^{(1) \mu \nu n}+\operatorname{Tr}\left(\nabla_{\mu} G^{-1} \nabla^{\mu} G\right)\right], \\
\delta g_{\mu \nu}= & \frac{1}{4}\left[2 F_{\mu \rho}^{(1) m} G_{m n} F_{\nu}^{(1) \rho n}-\operatorname{Tr}\left(\nabla_{(\mu} G^{-1} \nabla_{\nu)} G\right)\right], \\
\delta B_{\mu \nu}= & \frac{1}{8}\left(-2 \nabla^{\rho} F_{\rho \mu}^{(1) m}+2 \nabla^{\rho} \Phi F_{\rho \mu}^{(1) m}+H_{\mu \rho \sigma} H^{\rho \sigma}{ }_{p} G^{p m}+F_{\mu \rho}^{(1) p}\left(\nabla^{\rho} G G^{-1}\right)_{p}{ }^{m}\right. \\
& \left.+2 H_{\mu \rho p}\left(G^{-1} \nabla^{\rho} B G^{-1}\right)^{p m}\right)\left(A_{\nu m}^{(2)}-B_{m n} A_{\nu}^{(1) n}\right)-(\mu \leftrightarrow \nu), \\
\delta G^{m n}= & \frac{1}{4}\left[-2 \square G^{m n}+2 \nabla_{\mu} \Phi \nabla^{\mu} G^{m n}-G^{m p} H_{\mu \nu p} G^{n q} H^{\mu \nu}{ }_{q}-\frac{3}{2} F_{\mu \nu}^{(1) m} F^{(1) \mu \nu n}\right. \\
& \left.-\left(G^{-1} \nabla_{\mu} G \nabla^{\mu} G^{-1}\right)^{m n}+2\left(G^{-1} \nabla_{\mu} B G^{-1} \nabla^{\mu} B G^{-1}\right)^{m n}\right],
\end{aligned}
$$




$$
\begin{aligned}
\delta A_{\mu}^{(1) m}= & \frac{1}{4}\left[-2 \nabla^{\nu} F_{\nu \mu}^{(1) m}+2 \nabla^{\nu} \Phi F_{\nu \mu}^{(1) m}+H_{\mu \nu \rho} H^{\nu \rho}{ }_{n} G^{n m}+F_{\mu \nu}^{(1) n}\left(\nabla^{\nu} G G^{-1}\right)_{n}{ }^{m}\right. \\
& \left.+2 H_{\mu \nu n}\left(G^{-1} \nabla^{\nu} B G^{-1}\right)^{n m}\right], \\
\delta A_{\mu m}^{(2)}= & \frac{1}{4}\left[2 \nabla^{\nu} F_{\nu \mu}^{(1) n} B_{n m}-2 \nabla^{\nu} \Phi F_{\nu \mu}^{(1) n} B_{n m}-H_{\mu \nu \rho} H^{\nu \rho}{ }_{n}\left(G^{-1} B\right)^{n}{ }_{m}-F_{\mu \nu}^{(1) n}\left(\nabla^{\nu} G G^{-1} B\right)_{n m}\right. \\
& \left.-2 H_{\mu \nu n}\left(G^{-1} \nabla^{\nu} B G^{-1} B\right)^{n}{ }_{m}\right] .
\end{aligned}
$$

\section{2. $\hat{\boldsymbol{R}}_{\hat{\boldsymbol{\mu}} \hat{\nu} \hat{\rho} \hat{\boldsymbol{\sigma}}} \hat{\boldsymbol{H}}^{\hat{\mu} \hat{\nu} \hat{\lambda}} \hat{\boldsymbol{H}}^{\hat{\rho} \hat{\boldsymbol{\sigma}}_{\hat{\lambda}}}$}

Here, we consider the four last terms in the reduction (4.12) of $R H H$. After partial integration, they can be brought into the form

$$
\begin{aligned}
& \frac{\alpha^{\prime}}{4} \int \mathrm{d}^{D} x \sqrt{-g} e^{-\Phi} \operatorname{Tr}\left(\nabla_{\mu} \nabla_{\nu} G^{-1} \nabla^{\mu} B G^{-1} \nabla^{\nu} B\right) \\
& =\frac{\alpha^{\prime}}{4} \int \mathrm{d}^{D} x \sqrt{-g} e^{-\Phi}\left[-\operatorname{Tr}\left(\nabla_{\mu} B \nabla^{\mu} G^{-1} \nabla_{\nu} B \nabla^{\nu} G^{-1}\right)+\frac{1}{2} \operatorname{Tr}\left(\nabla_{\mu} B \nabla_{\nu} G^{-1} \nabla^{\mu} B \nabla^{\nu} G^{-1}\right)\right. \\
& \left.-\operatorname{Tr}\left(\left(\square B-\nabla_{\mu} \Phi \nabla^{\mu} B\right) G^{-1} \nabla_{\nu} B \nabla^{\nu} G^{-1}\right)+\frac{1}{2} \operatorname{Tr}\left(\left(\square G^{-1}-\nabla_{\mu} \Phi \nabla^{\mu} G^{-1}\right) \nabla_{\nu} B G^{-1} \nabla^{\nu} B\right)\right], \\
& -\frac{\alpha^{\prime}}{4} \int \mathrm{d}^{D} x \sqrt{-g} e^{-\Phi} H_{m}^{\mu \rho} \nabla_{\mu} \nabla_{\nu} G^{m n} H_{\rho n}^{\nu} \\
& =\frac{\alpha^{\prime}}{4} \int \mathrm{d}^{D} x \sqrt{-g} e^{-\Phi}\left[-\left(\nabla_{\mu} H^{\mu \nu}{ }_{m}-\nabla_{\mu} \Phi H^{\mu \nu}{ }_{m}\right) \nabla^{\rho} G^{m n} H_{\nu \rho n}-\frac{1}{2} F_{\mu \nu}^{(1) m}\left(\nabla_{\rho} B \nabla^{\rho} G^{-1}\right)_{m}{ }^{n} H^{\mu \nu}{ }_{n}\right. \\
& \left.-\frac{1}{4} H_{m}^{\mu \nu}\left(\square G^{-1}-\nabla_{\rho} \Phi \nabla^{\rho} G^{-1}\right)^{m n} H_{\mu \nu n}-F_{\nu \rho}^{(1) m}\left(\nabla_{\mu} B \nabla^{\rho} G^{-1}\right)_{m}{ }^{n} H^{\mu \nu}{ }_{n}\right] \text {, } \\
& -\frac{\alpha^{\prime}}{2} \int \mathrm{d}^{D} x \sqrt{-g} e^{-\Phi} \nabla_{\mu} F_{\nu \rho}^{(1) m}\left(\nabla^{\rho} B G^{-1}\right)_{m}{ }^{n} H^{\mu \nu}{ }_{n} \\
& =\frac{\alpha^{\prime}}{4} \int \mathrm{d}^{D} x \sqrt{-g} e^{-\Phi}\left[-\left(\nabla_{\mu} H^{\mu \nu}{ }_{m}-\nabla_{\mu} \Phi H^{\mu \nu}{ }_{m}\right)\left(G^{-1} \nabla^{\rho} B\right)^{m}{ }_{n} F_{\nu \rho}^{(1) n}\right. \\
& -\left(\nabla_{\mu} F^{(1) \mu \nu m}-\nabla_{\mu} \Phi F^{(1) \mu \nu m}\right)\left(\nabla^{\rho} B G^{-1}\right)_{m}{ }^{n} H_{\nu \rho n}+\frac{1}{2} F_{\mu \nu}^{(1) m}\left(\nabla_{\rho} B G^{-1} \nabla^{\rho} B\right)_{m n} F^{(1) \mu \nu n} \\
& -\frac{1}{2} F_{\mu \nu}^{(1) m}\left(\nabla_{\rho} B \nabla^{\rho} G^{-1}\right)_{m}{ }^{n} H^{\mu \nu}{ }_{n}-\frac{1}{2} F_{\mu \nu}^{(1) m}\left(\left(\square B-\nabla_{\rho} \Phi \nabla^{\rho} B\right) G^{-1}\right)_{m}{ }^{n} H^{\mu \nu}{ }_{n} \\
& +F_{\nu \rho}^{(1) m}\left(\nabla^{\rho} B \nabla_{\mu} G^{-1}\right)_{m}{ }^{n} H^{\mu \nu}{ }_{n}-F_{\nu \rho}^{(1) m}\left(\nabla_{\mu} B \nabla^{\rho} G^{-1}\right)_{m}{ }^{n} H^{\mu \nu}{ }_{n} \\
& \left.+F_{\mu \nu}^{(1) m}\left(\nabla_{\rho} B G^{-1} \nabla^{\nu} B\right)_{m n} F^{(1) \rho \mu n}\right] \text {, } \\
& \frac{\alpha^{\prime}}{4} \int \mathrm{d}^{D} x \sqrt{-g} e^{-\Phi} 2 H^{\mu \nu \lambda} \nabla_{\mu} F_{\nu \rho}^{(1) m} H_{\lambda m}^{\rho} \\
& =\frac{\alpha^{\prime}}{4} \int \mathrm{d}^{D} x \sqrt{-g} e^{-\Phi}\left[\frac{1}{2}\left(\nabla_{\mu} H^{\mu \nu}{ }_{m}-\nabla_{\mu} \Phi H^{\mu \nu}{ }_{m}\right) H_{\nu \rho \sigma} F^{(1) \rho \sigma m}-H^{\mu \nu \rho} F_{\nu \sigma}^{(1) m} \nabla_{\mu} B_{m n} F_{\rho}^{(1) \sigma n}\right. \\
& -\left(\nabla_{\mu} H^{\mu \nu \rho}-\nabla_{\mu} \Phi H^{\mu \nu \rho}\right) F_{\nu \sigma}^{(1) m} H_{\rho m}^{\sigma}+\frac{1}{2}\left(\nabla_{\mu} F^{(1) \mu \nu m}-\nabla_{\mu} \Phi F^{(1) \mu \nu m}\right) H_{\nu \rho \sigma} H^{\rho \sigma}{ }_{m} \\
& +\frac{1}{2} H^{\mu \nu \rho} F_{\nu \sigma}^{(1) m} \nabla^{\sigma} B_{m n} F_{\rho \mu}^{(1) n}+F_{\mu \nu}^{(1) m} H_{\rho \sigma m} F^{(1) \rho \nu n} H^{\nu \sigma}{ }_{n}-\frac{1}{4} F_{\mu \nu}^{(1) m} H_{\rho \sigma m} F^{(1) \mu \nu n} H^{\rho \sigma}{ }_{n} \\
& \left.-\frac{1}{4} F_{\mu \nu}^{(1) m} H_{\rho \sigma m} F^{(1) \rho \sigma n} H^{\mu \nu}{ }_{n}\right] \text {, }
\end{aligned}
$$


respectively. Again, all leftover terms carrying second-order derivatives can be converted to products of first-order derivatives by means of the rules of Table I. This induces the explicit field redefinitions

$$
\begin{aligned}
\delta B_{\mu \nu}= & \frac{1}{8}\left[-A_{\mu}^{(1) m}\left(G \nabla_{\rho} G^{-1}\right)_{m}{ }^{n} H_{\nu \rho n}-A_{\mu}^{(1) m} \nabla^{\rho} B_{m n} F_{\nu \rho}^{(1) n}+2 F_{\mu \rho}^{(1) m} H^{\rho}{ }_{\nu m}\right. \\
& +\frac{1}{2} A_{\mu}^{(1) m} H_{\nu \rho \sigma} G_{m n} F^{(1) \rho \sigma n}-\frac{1}{2} H_{\mu \rho \sigma} H^{\rho \sigma}{ }_{m} G^{m n}\left(A_{\nu n}^{(2)}-B_{n p} A_{\nu}^{(1) p}\right) \\
& \left.-H_{\mu \rho m}\left(G^{-1} \nabla^{\rho} B G^{-1}\right)^{m n}\left(A_{\nu n}^{(2)}-B_{n p} A_{\nu}^{(1) p}\right)\right]-(\mu \leftrightarrow \nu), \\
\delta G^{m n}= & \frac{1}{4}\left[\frac{1}{2} G^{m p} H_{\mu \nu p} G^{n q} H^{\mu \nu}{ }_{q}-\left(G^{-1} \nabla_{\mu} B G^{-1} \nabla^{\mu} B G^{-1}\right)^{m n}\right], \\
\delta B_{m n}= & \frac{1}{4}\left[\left(\nabla_{\mu} B \nabla^{\mu} G^{-1} G\right)_{m n}+\left(G \nabla_{\mu} G^{-1} \nabla^{\mu} B\right)_{m n}\right. \\
& \left.-\frac{1}{2} H_{\mu \nu m} F^{(1) \mu \nu p} G_{p n}+\frac{1}{2} G_{m p} F^{(1) \mu \nu p} H_{\mu \nu n}\right], \\
\delta A_{\mu}^{(1) m}= & \frac{1}{4}\left[-H_{\mu \nu n}\left(G^{-1} \nabla^{\nu} B G^{-1}\right)^{n m}-\frac{1}{2} H_{\mu \nu \rho} H^{\nu \rho}{ }_{n} G^{n m}\right], \\
\delta A_{\mu m}^{(2)}= & \frac{1}{4}\left[H_{\mu \nu n}\left(\nabla^{\nu} G^{-1} G\right)^{n}{ }_{m}-F_{\mu \nu}^{(1) n} \nabla^{\nu} B_{n m}+H_{\mu \nu n}\left(G^{-1} \nabla^{\nu} B G^{-1} B\right)^{n}{ }_{m}\right. \\
& \left.-\frac{1}{2} H_{\mu \nu \rho} F^{(1) \nu \rho n} G_{n m}+\frac{1}{2} H_{\mu \nu \rho} H^{\nu \rho}{ }_{n}\left(G^{-1} B\right)^{n}{ }_{m}\right] .
\end{aligned}
$$

\section{3. $\hat{\boldsymbol{H}}^{\hat{\mu} \hat{\nu} \hat{\rho}} \hat{\mathbf{\Omega}}_{\hat{\mu} \hat{\nu} \hat{\rho}}^{(\hat{\rho})}$}

Finally, we give the result for the last six terms in Eq. (7.8). After partial integration they are rewritten as

$$
\begin{gathered}
\frac{\alpha^{\prime}}{4} \int \mathrm{d}^{D} x \sqrt{-g} e^{-\Phi}\left(-\frac{1}{2}\right) H_{m}^{\mu \nu} G^{m n} \nabla_{\mu}\left(\nabla_{\nu} e_{\sigma}{ }^{\alpha} e_{\alpha}{ }^{\rho} F_{\rho}^{(1) \sigma p} G_{n p}\right) \\
=\frac{\alpha^{\prime}}{4} \int \mathrm{d}^{D} x \sqrt{-g} e^{-\Phi}\left[\frac{1}{2}\left(\nabla_{\mu} H^{\mu \nu}{ }_{p}-\nabla_{\mu} \Phi H^{\mu \nu}{ }_{p}+H^{\mu \nu}{ }_{m} \nabla_{\mu} G^{m n} G_{n p}\right) \nabla_{\nu} e_{\sigma}{ }^{\alpha} e_{\alpha}{ }^{\rho} F_{\rho}^{(1) \sigma p}\right], \\
\frac{\alpha^{\prime}}{4} \int \mathrm{d}^{D} x \sqrt{-g} e^{-\Phi} \frac{1}{2} \operatorname{Tr}\left(\nabla_{\mu} \nabla_{\nu} G \nabla^{\mu} G^{-1} \nabla^{\nu} B G^{-1}\right) \\
=\frac{\alpha^{\prime}}{4} \int \mathrm{d}^{D} x \sqrt{-g} e^{-\Phi}\left[-\frac{1}{2} \operatorname{Tr}\left(\left(\square B-\nabla_{\mu} \Phi \nabla^{\mu} B\right) G^{-1} \nabla_{\nu} G \nabla^{\nu} G^{-1}\right)\right. \\
\left.+\frac{1}{2} \operatorname{Tr}\left(\left(\square G-\nabla_{\mu} \Phi \nabla^{\mu} G\right) \nabla_{\nu} G^{-1} \nabla^{\nu} B G^{-1}\right)-\operatorname{Tr}\left(\nabla_{\mu} B \nabla^{\mu} G^{-1} \nabla_{\nu} G \nabla^{\nu} G^{-1}\right)\right], \\
\frac{\alpha^{\prime}}{4} \int \mathrm{d}^{D} x \sqrt{-g} e^{-\Phi}\left[-\frac{1}{2} H_{\mu \nu m}\left(G^{-1} \nabla_{\rho} G\right)^{m}{ }_{n} \nabla^{\mu} F^{(1) \nu \rho n}+\frac{1}{2} H_{\mu \nu m}\left(G^{-1} \nabla^{\mu} \nabla_{\rho} G\right)^{m}{ }_{n} F^{(1) \nu \rho n}\right] \\
=\frac{\alpha^{\prime}}{4} \int \mathrm{d}^{D} x \sqrt{-g} e^{-\Phi}\left[-\frac{1}{2}\left(\nabla_{\mu} F^{(1) \mu \nu m}-\nabla_{\mu} \Phi F^{(1) \mu \nu m}\right)\left(\nabla^{\rho} G G^{-1}\right)_{m}{ }^{n} H_{\nu \rho n}\right. \\
-\frac{1}{4} H_{\mu \nu m}\left(G^{-1}\left(\square G-\nabla_{\rho} \Phi \nabla^{\rho} G+\nabla_{\rho} G \nabla^{\rho} G^{-1} G\right)\right)^{m}{ }_{n} F^{(1) \mu \nu n}-\frac{1}{2} H_{\nu \rho m}\left(\nabla_{\mu} G^{-1} \nabla^{\rho} G\right)^{m}{ }_{n} F^{(1) \mu \nu n} \\
\left.-\frac{1}{2} F_{\nu \rho}^{(1) m}\left(\nabla_{\mu} B G^{-1} \nabla^{\rho} G\right)_{m n} F^{(1) \mu \nu n}-\frac{1}{4} F_{\mu \nu}^{(1) m}\left(\nabla_{\rho} B G^{-1} \nabla^{\rho} G\right)_{m n} F^{(1) \mu \nu n}\right],
\end{gathered}
$$




$$
\begin{aligned}
& \frac{\alpha^{\prime}}{4} \int \mathrm{d}^{D} x \sqrt{-g} e^{-\Phi} \frac{1}{2} H^{\mu \nu \rho} F_{\mu \sigma}^{(1) m} G_{m n} \nabla_{\nu} F_{\rho}^{(1) \sigma n} \\
& =\frac{\alpha^{\prime}}{4} \int \mathrm{d}^{D} x \sqrt{-g} e^{-\Phi}\left[-\frac{1}{4} H_{\mu \nu \rho}\left(\nabla_{\sigma} F^{(1) \sigma \mu m}-\nabla_{\sigma} \Phi F^{(1) \sigma \mu m}+F^{(1) \sigma \mu p}\left(\nabla_{\sigma} G G^{-1}\right)_{p}{ }^{m}\right) G_{m n} F_{\nu \rho}^{(1) n}\right. \\
& \left.-\frac{1}{4} F_{\mu \nu}^{(1) m} H_{\rho \sigma m} F^{(1) \mu \rho p} G_{p q} F^{(1) \nu \sigma q}+\frac{1}{8} F_{\mu \nu}^{(1) m} H_{\rho \sigma m} F^{(1) \mu \nu p} G_{p q} F^{(1) \rho \sigma q}\right] \\
& \frac{\alpha^{\prime}}{4} \int \mathrm{d}^{D} x \sqrt{-g} e^{-\Phi}\left(-\frac{1}{4}\right) F_{\mu \nu}^{(1) m} \nabla_{\rho} B_{m n} \nabla^{\rho} F^{(1) \mu \nu n} \\
& =\frac{\alpha^{\prime}}{4} \int \mathrm{d}^{D} x \sqrt{-g} e^{-\Phi}\left[-\frac{1}{2}\left(\nabla_{\mu} F^{(1) \mu \nu m}-\nabla_{\mu} \Phi F^{(1) \mu \nu m}\right) \nabla^{\rho} B_{m n} F_{\nu \rho}^{(1) n}\right] .
\end{aligned}
$$

Again, the remaining terms carrying second-order derivatives can be eliminated by field redefinitions as discussed in Sec. II B. The explicit form of the induced field redefinitions has been given in Eq. (7.10) in the main text.

\section{APPENDIX C: GL $(d)$ EXPRESSIONS OF SOME O $(d, d, \mathbb{R})$ TERMS}

In this Appendix, we present the $\mathrm{GL}(d)$ decomposition of some of the $\mathrm{O}(d, d, \mathbb{R})$ invariant terms that are relevant for the identifications made in Secs. V and VII.

$$
\begin{aligned}
& \operatorname{Tr}\left(\nabla_{\mu} \mathcal{S} \nabla_{\nu} \mathcal{S}\right)= 2 \operatorname{Tr}\left(\nabla_{(\mu} G \nabla_{\nu} G^{-1}\right)+2 \operatorname{Tr}\left(\nabla_{\mu} B G^{-1} \nabla_{\nu} B G^{-1}\right), \\
& \operatorname{Tr}\left(\nabla_{\mu} \mathcal{S} \nabla_{\nu} \mathcal{S} \nabla^{\mu} \mathcal{S} \nabla^{\nu} \mathcal{S}\right)= \operatorname{Tr}\left(\nabla_{\mu} G^{-1} \nabla_{\nu} G \nabla^{\mu} G^{-1} \nabla^{\nu} G\right)+4 \operatorname{Tr}\left(\nabla_{\mu} B \nabla_{\nu} G^{-1} \nabla^{\mu} B \nabla^{\nu} G^{-1}\right) \\
&+8 \operatorname{Tr}\left(G^{-1} \nabla_{\mu} B G^{-1} \nabla_{\nu} G \nabla^{\mu} G^{-1} \nabla^{\nu} B\right) \\
&+2 \operatorname{Tr}\left(G^{-1} \nabla_{\mu} B G^{-1} \nabla_{\nu} B G^{-1} \nabla^{\mu} B G^{-1} \nabla^{\nu} B\right), \\
& \operatorname{Tr}\left(\nabla_{\mu} \mathcal{S} \nabla^{\mu} \mathcal{S} \nabla_{\nu} \mathcal{S} \nabla^{\nu} \mathcal{S}\right)= 2 \operatorname{Tr}\left(\nabla_{\mu} G^{-1} \nabla^{\mu} G \nabla_{\nu} G^{-1} \nabla^{\nu} G\right)+4 \operatorname{Tr}\left(G^{-1} \nabla_{\mu} B G^{-1} \nabla_{\nu} G \nabla^{\nu} G^{-1} \nabla^{\mu} B\right) \\
&+4 \operatorname{Tr}\left(\nabla_{\mu} G^{-1} \nabla^{\mu} B \nabla_{\nu} G^{-1} \nabla^{\nu} B\right)+4 \operatorname{Tr}\left(G^{-1} \nabla_{\mu} B \nabla^{\mu} G^{-1} \nabla_{\nu} G G^{-1} \nabla^{\nu} B\right) \\
&+2 \operatorname{Tr}\left(G^{-1} \nabla_{\mu} B G^{-1} \nabla^{\mu} B G^{-1} \nabla_{\nu} B G^{-1} \nabla^{\nu} B\right), \\
& \operatorname{Tr}\left(\mathcal{S} \nabla_{\mu} \mathcal{S} \nabla_{\nu} \mathcal{S} \nabla_{\rho} \mathcal{S} \nabla_{\sigma} \mathcal{S}\right)=2\left[\operatorname{Tr}\left(\nabla_{\mu} G^{-1} \nabla_{\nu} G \nabla_{\rho} G^{-1} \nabla_{\sigma} B\right)-\operatorname{Tr}\left(\nabla_{\sigma} G^{-1} \nabla_{\mu} G \nabla_{\nu} G^{-1} \nabla_{\rho} B\right)\right. \\
&+ \operatorname{Tr}\left(\nabla_{\rho} G^{-1} \nabla_{\sigma} G \nabla_{\mu} G^{-1} \nabla_{\nu} B\right)-\operatorname{Tr}\left(\nabla_{\nu} G^{-1} \nabla_{\rho} G \nabla_{\sigma} G^{-1} \nabla_{\mu} B\right) \\
&- \operatorname{Tr}\left(\nabla_{\mu} B G^{-1} \nabla_{\nu} B G^{-1} \nabla_{\rho} B \nabla_{\sigma} G^{-1}\right)+\operatorname{Tr}\left(\nabla_{\sigma} B G^{-1} \nabla_{\mu} B G^{-1} \nabla_{\nu} B \nabla_{\rho} G^{-1}\right) \\
&-\left.\operatorname{Tr}\left(\nabla_{\rho} B G^{-1} \nabla_{\sigma} B G^{-1} \nabla_{\mu} B \nabla_{\nu} G^{-1}\right)+\operatorname{Tr}\left(\nabla_{\nu} B G^{-1} \nabla_{\rho} B G^{-1} \nabla_{\sigma} B \nabla_{\mu} G^{-1}\right)\right], \\
& \mathcal{F}_{\mu \nu}{ }^{M} \mathcal{S}_{M}{ }^{N} \mathcal{F}_{\rho \sigma N}=F_{\mu \nu}^{(1) m} G_{m n} F_{\rho \sigma}^{(1) n}+H_{\mu \nu m} G^{m n} H_{\rho \sigma n}, \\
& \mathcal{F}_{\mu \nu}{ }^{M} \mathcal{F}_{\rho \sigma M}=F_{\mu \nu}^{(1) m} F_{\rho \sigma m}^{(2)}+F_{\rho \sigma}^{(1) m} F_{\mu \nu m}^{(2)}, \\
& \mathcal{F}_{\mu \nu}{ }^{M} \mathcal{S}_{M}{ }^{N} \nabla_{\rho} \mathcal{S}_{N}{ }^{P} \mathcal{F}_{\sigma \lambda P}= F_{\mu \nu}^{(1) m}\left(G \nabla_{\rho} G^{-1}\right)_{m}{ }^{n} H_{\rho \lambda n}-F_{\mu \nu}^{(1) m} \nabla_{\rho} B_{m n} F_{\sigma \lambda}^{(1) n} \\
&+H_{\mu \nu m}\left(G^{-1} \nabla_{\rho} B G^{-1}\right)^{m n} H_{\sigma \lambda n}+H_{\mu \nu m}\left(G^{-1} \nabla_{\rho} G\right)^{m}{ }_{n} F_{\sigma \lambda}^{(1) n}, \\
& \mathcal{F}_{\mu \nu}{ }^{M} \nabla_{\rho} \mathcal{S}_{M}{ }^{N} \mathcal{F}_{\sigma \lambda N}=F_{\mu \nu}^{(1) m} \nabla_{\rho} G_{m n} F_{\sigma \lambda}^{(1) n}+F_{\mu \nu}^{(1) m}\left(\nabla_{\rho} B G^{-1}\right)_{m}{ }^{n} H_{\rho \lambda n} \\
&-H_{\mu \nu m}\left(G^{-1} \nabla_{\rho} B\right)^{m}{ }_{n} F_{\sigma \lambda}^{(1) n}+H_{\mu \nu m} \nabla_{\rho} G^{m n} H_{\sigma \lambda n},
\end{aligned}
$$




$$
\begin{aligned}
\mathcal{F}_{\mu \nu}{ }^{M} \mathcal{S}_{M}{ }^{N} \nabla_{\rho} \mathcal{S}_{N}{ }^{P} \nabla_{\sigma} \mathcal{S}_{P} Q \mathcal{F}_{\lambda \tau Q}= & F_{\mu \nu}^{(1) m}\left(G \nabla_{\rho} G^{-1} \nabla_{\sigma} G\right)_{m n} F_{\lambda \tau}^{(1) n}+F_{\mu \nu}^{(1) m}\left(G \nabla_{\rho} G^{-1} \nabla_{\sigma} B G^{-1}\right)_{m}{ }^{n} H_{\lambda \tau n} \\
& -F_{\mu \nu}^{(1) m}\left(\nabla_{\rho} B \nabla_{\sigma} G^{-1}\right)_{m}{ }^{n} H_{\lambda \tau n}+H_{\mu \nu m}\left(\nabla_{\rho} G^{-1} \nabla_{\sigma} B\right)^{m}{ }_{n} F_{\lambda \tau}^{(1) n} \\
& +H_{\mu \nu m}\left(G^{-1} \nabla_{\rho} B G^{-1} \nabla_{\sigma} G\right)^{m}{ }_{n} F_{\lambda \tau}^{(1) n}+F_{\mu \nu}^{(1) m}\left(\nabla_{\rho} B G^{-1} \nabla_{\sigma} B\right)_{m n} F_{\lambda \tau}^{(1) n} \\
& +H_{\mu \nu m}\left(G^{-1} \nabla_{\rho} G \nabla_{\sigma} G^{-1}\right)^{m n} H_{\lambda \tau n}+H_{\mu \nu m}\left(G^{-1} \nabla_{\rho} B G^{-1} \nabla_{\sigma} B G^{-1}\right)^{m n} H_{\lambda \tau n}, \\
\mathcal{F}_{\mu \nu}{ }^{M} \nabla_{\rho} \mathcal{S}_{M}{ }^{N} \nabla_{\sigma} \mathcal{S}_{N}{ }^{P} \mathcal{F}_{\lambda \tau P}= & F_{\mu \nu}^{(1) m}\left(\nabla_{\rho} B G^{-1} \nabla_{\sigma} G\right)_{m n} F_{\lambda \tau}^{(1) n}-F_{\mu \nu}^{(1) m}\left(\nabla_{\rho} G G^{-1} \nabla_{\sigma} B\right)_{m n} F_{\lambda \tau}^{(1) n} \\
& +F_{\mu \nu}^{(1) m}\left(\nabla_{\rho} G \nabla_{\sigma} G^{-1}\right)_{m}{ }^{n} H_{\lambda \tau n}+F_{\mu \nu}^{(1) m}\left(\nabla_{\rho} B G^{-1} \nabla_{\sigma} B G^{-1}\right)_{m}{ }^{n} H_{\lambda \tau n} \\
& +H_{\mu \nu m}\left(\nabla_{\rho} G^{-1} \nabla_{\sigma} G\right)^{m}{ }_{n} F_{\lambda \tau}^{(1) n}+H_{\mu \nu m}\left(G^{-1} \nabla_{\rho} B G^{-1} \nabla_{\sigma} B\right)^{m}{ }_{n} F_{\lambda \tau}^{(1) n} \\
& +H_{\mu \nu m}\left(\nabla_{\rho} G^{-1} \nabla_{\sigma} B G^{-1}\right)^{m n} H_{\lambda \tau n}-H_{\mu \nu m}\left(G^{-1} \nabla_{\rho} B \nabla_{\sigma} G^{-1}\right)^{m n} H_{\lambda \tau n} .
\end{aligned}
$$

[1] G. Veneziano, Scale factor duality for classical and quantum strings, Phys. Lett. B 265, 287 (1991).

[2] K. Meissner and G. Veneziano, Symmetries of cosmological superstring vacua, Phys. Lett. B 267, 33 (1991).

[3] J. Maharana and J. H. Schwarz, Noncompact symmetries in string theory, Nucl. Phys. B390, 3 (1993).

[4] A. Sen, $O(d) \otimes O(d)$ symmetry of the space of cosmological solutions in string theory, scale factor duality and two-dimensional black holes, Phys. Lett. B 271, 295 (1991).

[5] K. A. Meissner, Symmetries of higher order string gravity actions, Phys. Lett. B 392, 298 (1997).

[6] E. Bergshoeff, B. Janssen, and T. Ortin, Solution generating transformations and the string effective action, Classical Quantum Gravity 13, 321 (1996).

[7] N. Kaloper and K. A. Meissner, Duality beyond the first loop, Phys. Rev. D 56, 7940 (1997).

[8] H. Godazgar and M. Godazgar, Duality completion of higher derivative corrections, J. High Energy Phys. 09 (2013) 140.

[9] T. Kugo and B. Zwiebach, Target space duality as a symmetry of string field theory, Prog. Theor. Phys. 87, 801 (1992).

[10] O. Hohm and B. Zwiebach, T-duality constraints on higher derivatives revisited, J. High Energy Phys. 04 (2016) 101.

[11] O. Hohm and B. Zwiebach, Duality invariant cosmology to all orders in $\alpha^{\prime}$, Phys. Rev. D 100, 126011 (2019).

[12] W. Siegel, Superspace duality in low-energy superstrings, Phys. Rev. D 48, 2826 (1993).

[13] C. Hull and B. Zwiebach, Double field theory, J. High Energy Phys. 09 (2009) 099.

[14] O. Hohm, C. Hull, and B. Zwiebach, Background independent action for double field theory, J. High Energy Phys. 07 (2010) 016.

[15] O. Hohm, C. Hull, and B. Zwiebach, Generalized metric formulation of double field theory, J. High Energy Phys. 08 (2010) 008.
[16] O. Hohm, W. Siegel, and B. Zwiebach, Doubled $\alpha^{\prime}$-geometry, J. High Energy Phys. 02 (2014) 065.

[17] O. Hohm and B. Zwiebach, Green-Schwarz mechanism and $\alpha^{\prime}$-deformed Courant brackets, J. High Energy Phys. 01 (2015) 012.

[18] O. Hohm and B. Zwiebach, Double field theory at order $\alpha^{\prime}$, J. High Energy Phys. 11 (2014) 075.

[19] D. Marques and C. A. Nunez, T-duality and $\alpha^{\prime}$-corrections, J. High Energy Phys. 10 (2015) 084.

[20] E. Lescano and D. Marques, Second order higher-derivative corrections in double field theory, J. High Energy Phys. 06 (2017) 104.

[21] U. Naseer and B. Zwiebach, Three-point functions in duality-invariant higher-derivative gravity, J. High Energy Phys. 03 (2016) 147.

[22] W. H. Baron, J. J. Fernandez-Melgarejo, D. Marques, and C. Nunez, The odd story of $\alpha^{\prime}$-corrections, J. High Energy Phys. 04 (2017) 078.

[23] W. H. Baron, E. Lescano, and D. Marqués, The generalized Bergshoeff-de Roo identification, J. High Energy Phys. 11 (2018) 160.

[24] O. Hohm, Background Independence and Duality Invariance in String Theory, Phys. Rev. Lett. 118, 131601 (2017).

[25] O. Hohm, Background independent double field theory at order $\alpha^{\prime}$ : Metric vs. frame-like geometry, Phys. Rev. D 95, 066018 (2017).

[26] C. Eloy, O. Hohm, and H. Samtleben, Green-Schwarz Mechanism for String Dualities, Phys. Rev. Lett. 124, 091601 (2020).

[27] O. Hohm, A. Sen, and B. Zwiebach, Heterotic effective action and duality symmetries revisited, J. High Energy Phys. 02 (2015) 079.

[28] G. Pólya and R. C. Read, Combinatorial Enumeration of Groups, Graphs, and Chemical Compounds (SpringerVerlag, Berlin, 1987).

[29] J. F. Morales and H. Samtleben, Higher spin holography for SYM in $d$ dimensions, Phys. Lett. B 607, 286 (2005). 
[30] R. R. Metsaev and A. A. Tseytlin, Order $\alpha^{\prime}$ (two loop) equivalence of the string equations of motion and the $\sigma$-model Weyl invariance conditions: Dependence on the dilaton and the antisymmetric tensor, Nucl. Phys. B293, 385 (1987).

[31] L. Bao, J. Bielecki, M. Cederwall, B. E. W. Nilsson, and D. Persson, U-duality and the compactified Gauss-Bonnet term, J. High Energy Phys. 07 (2008) 048.

[32] G. Ferretti and S. Rajeev, Current Algebra in ThreeDimensions, Phys. Rev. Lett. 69, 2033 (1992).

[33] E. D'Hoker, Invariant effective actions, cohomology of homogeneous spaces and anomalies, Nucl. Phys. B451, 725 (1995).

[34] M. Gasperini, J. Maharana, and G. Veneziano, From trivial to nontrivial conformal string backgrounds via $\mathrm{O}(d, d)$ transformations, Phys. Lett. B 272, 277 (1991).

[35] A. Ali and A. Kumar, $O(d, d)$ transformations and $3-D$ black hole, Mod. Phys. Lett. A 08, 2045 (1993).

[36] P. A. Cano, P. Meessen, T. Ortín, and P. F. Ramírez, $\alpha^{\prime}$-corrected black holes in string theory, J. High Energy Phys. 05 (2018) 110.

[37] P. A. Cano, S. Chimento, P. Meessen, T. Ortín, P. F. Ramírez, and A. Ruipérez, Beyond the near-horizon limit:
Stringy corrections to heterotic black holes, J. High Energy Phys. 02 (2019) 192.

[38] S. Chimento, P. Meessen, T. Ortin, P. F. Ramirez, and A. Ruiperez, On a family of $\alpha^{\prime}$-corrected solutions of the heterotic superstring effective action, J. High Energy Phys. 07 (2018) 080.

[39] E. Bergshoeff and M. de Roo, The quartic effective action of the heterotic string and supersymmetry, Nucl. Phys. B328, 439 (1989).

[40] M. R. Garousi and H. Razaghian, Minimal independent couplings at order $\alpha^{\prime 2}$, Phys. Rev. D 100, 106007 (2019).

[41] M. R. Garousi, Effective action of bosonic string theory at order $\alpha^{\prime 2}$, Eur. Phys. J. C 79, 827 (2019).

[42] R. Borsato, A. V. López, and L. Wulff, The first $\alpha^{\prime}$-correction to homogeneous Yang-Baxter deformations using $O(d, d)$, arXiv:2003.05867.

[43] N. Lambert and P. C. West, Enhanced coset symmetries and higher derivative corrections, Phys. Rev. D 74, 065002 (2006).

[44] L. Bao, M. Cederwall, and B. E. W. Nilsson, Aspects of higher curvature terms and U-duality, Classical Quantum Gravity 25, 095001 (2008). 